\title{
Asymptotic solutions of Whittaker's differential equation as the moduli of the independent variable and two parameters tend to infinity
}

\author{
By Masahiro Iwano \\ (Received Sept. 14, 1963)
}

\section{§ 1. Introduction.}

In recent notes $[4, \mathbf{6}]$, we developed a general theory to construct asymptotic solutions of a system of linear ordinary differential equations containing a small parameter, and, by applying the theory to Bessel's differential equation

$$
t^{2} \frac{d^{2} y}{d t^{2}}+t \frac{d y}{d t}+\left(t^{2}-n^{2}\right) y=0
$$

we succeeded in constructing an asymptotic expression of a system of fundamental solutions when the moduli of the independent variable $t$ and parameter $n$ tend to infinity.

Our theory can be extended to the study of a linear ordinary differential equation containing two parameters with a singular point. In this note, we will show this by an example of Whittaker's differential equation

$$
\frac{d^{2} W}{d t^{2}}+\left[-\frac{1}{4}+\frac{k}{t}+\frac{\frac{1}{4}-n^{2}}{t^{2}}\right] W=0
$$

To do this, introducing a characteristic polyhedron (in place of a characteristic polygon in case of one parameter), we shall first solve the following problem :

1) Divide a domain

$$
\left\{k:|k|>R_{0}\right\} \times\left\{n:|n|>R_{1}\right\} \times\left\{t:|t|>R_{2}\right\}
$$

in $(k, n, t)$-space into a finite number of subdomains so that the solution behaves quite differently as $k, n, t$ tend to infinity in each of these subdomains.

Between the case of one parameter and the case of two parameters there exists an essential difference. In the former case such a division of the domain was uniquely determined by the aid of a characteristic polygon, while in the latter such a division of the domain is not uniquely determined in general (See §11).

Next, we shall solve the following problem:

2) Find out a complete set of asymptotic expression of independent solutions in each of these subdomains.

We shall see later that, in order to find out such a complete set, it is sufficient to study seven systems of certain normal types. 
To obtain the complete knowledge about the asymptotic behavior of the solutions of the given equation, we must solve the following problem:

3) Determine the so-called connection formula, i.e. a relation connecting two different complete sets of asymptotic expressions obtained in 2).

Unfortunately our efforts have failed to conquer the last problem which is perhaps the most important of these three problems. Concerning this, we should not forget to mention an important contribution to "asymptotic forms of Whittaker's function" by A. Erdélyi and C. A. Swanson [1]. They solved these three problems for Whittaker's differential equation under the restrictions that

i) $k$ is a large parameter;

ii) $n$ is fixed, real and non-negative;

iii) $t / k$ is real.

And, they obtained asymptotic forms in $x=t / k$ uniformly valid as $k$ tends to infinity.

Terminating the Introduction, the author wishes to express his cordial thanks to Professor Tosiya Saito for his kind guidance and valuable advice.

\section{$\S 2$. Transformation of Whittaker's differential equation.}

Since $k, n$ and $t$ are supposed to be sufficiently large, put

$$
\varepsilon=1 / k, \quad \tau=1 / n, \quad x=1 / t .
$$

Then, Whittaker's differential equation (1.2) becomes :

$$
\left.x^{2} \frac{d^{2} W}{d x^{2}}+2 x \frac{d W}{d x}+\left[\frac{1}{4 x^{2}}\left(x^{2}-1\right)+\frac{1}{\varepsilon x}-1\right] \tau^{2}\right] W=0 .
$$

By a transformation

$$
W=y / x,
$$

(W) will be reduced to

$$
x^{2} \frac{d^{2} y}{d x^{2}}+\left[\frac{1}{4 x^{2}}\left(x^{2}-1\right)+\frac{1}{\varepsilon x}-\frac{1}{\tau^{2}}\right] y=0 .
$$

We put further

$$
y_{1}=y, \quad y_{2}=\varepsilon^{\frac{1}{2} \tau} \frac{d y}{d x} .
$$

Then, we have a system of two differential equations

$$
\varepsilon^{\frac{1}{2} \tau} \frac{d \vec{y}}{d x}=\left(\begin{array}{cc}
0 & 1 \\
\varepsilon x^{-2}-\tau^{2} x^{-3}+\frac{1}{4} \varepsilon \tau^{2} x^{-4}\left(1-x^{2}\right) & 0
\end{array}\right) \vec{y} .
$$

Therefore, it is sufficient to study of the behavior of the solutions of the system $(\mathfrak{W})$ in a domain

$$
0<|\varepsilon| \leqq a_{0}, \quad 0<|\tau| \leqq b_{0}, \quad 0<|x| \leqq c_{0},
$$

where $a_{0}, b_{0}$ and $c_{0}$ are sufficiently small positive constants.

We explain the notations which will be frequently used in this note.

1) the letter with an arrow denotes a 2-dimensional column vector; 
2) the size of the matrix is 2 by 2 ;

3) the parameter and the independent variable are both complex-valued;

4) the small letter appearing in the inequalities defining a subdomain in a (complex) three dimensional product space is supposed to be sufficiently small, while the capital letter is supposed to be sufficiently large.

\section{Chapter I. Asymptotic series.}

\section{§3. Formal series.}

Before going into the definition of the characteristic polyhedron of the equation (WB), we explain the construction of an asymptotic series in three variables which will be regarded as an extention of the asymptotic series introduced by $\mathrm{K}$. Takahasi [7] in the case of two variables.

Let there be given a formal power series

$$
f(\varepsilon, \tau, x) \sim \sum_{j=0}^{\infty} \varepsilon^{p_{j} \tau^{q} j} x^{r} f_{j},
$$

where we suppose that

1) $f_{j}$ are constants ;

2) a set of the points $\mathrm{P}_{j}$ defined by $\left(p_{j}, q_{j}, r_{j}\right)$ forms a denumerably infinite set in $(X, Y, Z)$-space and has no finite limiting point;

3 ) this set is contained in a trigonal pyramid containing the first octant of $(X, Y, Z)$-space ${ }^{1)}$ in its interior and whose vertex is at the origin.

\section{$\S 4$. Trigonal pyramid.}

Let the equations of the three sides of the trigonal pyramid above-mentioned be written as

$$
\mathrm{S}: \quad 0=A X+B Y+C Z
$$

$(4.1)^{\prime} \quad \mathrm{S}^{\prime}: 0=A^{\prime} X+B^{\prime} Y+C^{\prime} Z$;

(4.1) $)^{\prime \prime} \mathrm{S}^{\prime \prime}: 0=A^{\prime \prime} X+B^{\prime \prime} Y+C^{\prime \prime} Z$.

Then, by hypothesis, the sides of the trigonal pyramid can not intersect with the interior of the first octant of $(X, Y, Z)$-space. Hence, we can suppose

$$
\left\{\begin{array}{c}
A \geqq 0, \quad B \geqq 0, \quad C \geqq 0 ; \\
A^{\prime} \geqq 0, \quad B^{\prime} \geqq 0, \quad C^{\prime} \geqq 0 ; \\
A^{\prime \prime} \geqq 0, B^{\prime \prime} \geqq 0, C^{\prime \prime} \geqq 0 .
\end{array}\right.
$$

Clearly, we have

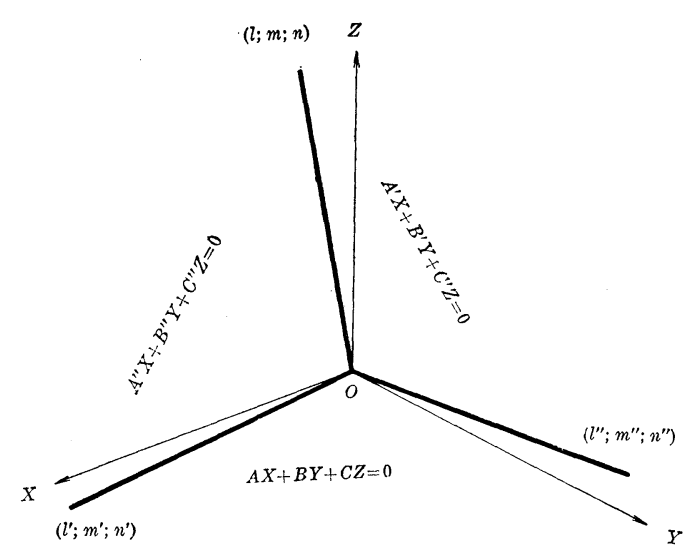

Fig. 1

$$
\Delta=\operatorname{det}\left|\begin{array}{lll}
A & B & C \\
A^{\prime} & B^{\prime} & C^{\prime} \\
A^{\prime \prime} & B^{\prime \prime} & C^{\prime \prime}
\end{array}\right| \neq 0 .
$$

1) The part of $(X, Y, Z)$-space such that $X \geqq 0, Y \geqq 0, Z \geqq 0$ will be called the first octant of $(X, Y, Z)$-space. 
Therefore, we can suppose, without loss of generality,

$$
\Delta>0 \text {. }
$$

Let $(l ; m ; n),\left(l^{\prime} ; m^{\prime} ; n^{\prime}\right)$ and $\left(l^{\prime \prime} ; m^{\prime \prime} ; n^{\prime \prime}\right)$ be directional parameters of the three edges $S^{\prime} \cap S^{\prime \prime}, S^{\prime \prime} \cap S$ and $S \cap S^{\prime}$ of the trigonal pyramid (See Fig. 1). Then, to simplify the description, we write this fact as

$$
(l ; m ; n),\left(l^{\prime} ; m^{\prime} ; n^{\prime}\right),\left(l^{\prime \prime} ; m^{\prime \prime} ; n^{\prime \prime}\right), \quad \quad \mathrm{S}^{\prime} \cup \mathrm{S}^{\prime \prime} \cup \mathrm{S}
$$

and an elementary calculation shows that:

$$
\begin{aligned}
& l: m: n \equiv\left(B^{\prime} C^{\prime \prime}-B^{\prime \prime} C^{\prime}\right):\left(C^{\prime} A^{\prime \prime}-C^{\prime \prime} A^{\prime}\right):\left(A^{\prime} B^{\prime \prime}-A^{\prime \prime} B^{\prime}\right) \text {, } \\
& l^{\prime}: m^{\prime}: n^{\prime} \equiv\left(B^{\prime \prime} C-B C^{\prime \prime}\right):\left(C^{\prime \prime} A-C A^{\prime \prime}\right):\left(A^{\prime \prime} B-A B^{\prime \prime}\right) \text {, } \\
& l^{\prime \prime}: m^{\prime \prime}: n^{\prime \prime} \equiv\left(B C^{\prime}-B^{\prime} C\right) \quad:\left(C A^{\prime}-C^{\prime} A\right) \quad:\left(A B^{\prime}-A^{\prime} B\right) \text {. }
\end{aligned}
$$

The right-hand members of $(4.1),(4.1)^{\prime},(4.1)^{\prime \prime}$ are all nonnegative for $X=X^{0}$, $Y=Y^{0}, Z=Z^{0}$, if the point $\left(X^{0}, Y^{0}, Z^{0}\right)$ does not lie outside the trigonal pyramid. Therefore, since the point

$$
X^{0}=p_{j}, \quad Y^{0}=q_{j}, \quad Z^{0}=r_{j}
$$

is contained in the trigonal pyramid, we have

$$
\begin{aligned}
\lambda(j) & \equiv A p_{j}+B q_{j}+C r_{j} \geqq 0, \\
\lambda^{\prime}(j) & \equiv A^{\prime} p_{j}+B^{\prime} q_{j}+C^{\prime} r_{j} \geqq 0, \\
\lambda^{\prime \prime}(j) & \equiv A^{\prime \prime} p_{j}+B^{\prime \prime} q_{j}+C^{\prime \prime} r_{j} \geqq 0 .
\end{aligned}
$$

On the other hand, each summand in the formal series (3.1) can be written as

$$
\varepsilon^{p_{j}} \tau^{q_{j}} x^{r_{j}}=\left(\varepsilon^{l} \tau^{m} x^{n}\right)^{\alpha_{j}}\left(\varepsilon^{l^{\prime}} \tau^{m^{\prime}} x^{n^{\prime}}\right)^{\beta}\left(\varepsilon^{l^{\prime \prime} \tau^{m^{\prime \prime}}} x^{n^{\prime \prime}}\right)^{\gamma_{j}}
$$

where $\alpha_{j}, \beta_{j}, \gamma_{j}$ are given by

$$
\begin{aligned}
& \left\{\begin{array}{l}
\alpha_{j}=\left[p_{j}\left(n^{\prime \prime} m^{\prime}-n^{\prime} m^{\prime \prime}\right)+q_{j}\left(l^{\prime \prime} n^{\prime}-l^{\prime} n^{\prime \prime}\right)+r_{j}\left(m^{\prime \prime} l^{\prime}-m^{\prime} l^{\prime \prime}\right)\right] / L, \\
\beta_{j}=\left[p_{j}\left(n m^{\prime \prime}-n^{\prime \prime} m\right)+q_{j}\left(\left(n^{\prime \prime}-l^{\prime \prime} n\right)+r_{j}\left(m l^{\prime \prime}-m^{\prime \prime} l\right)\right] / L,\right. \\
\gamma_{j}=\left[p_{j}\left(n^{\prime} m-n m^{\prime}\right)+q_{j}\left(l^{\prime} n-l n^{\prime}\right)+r_{j}\left(m^{\prime} l-m l^{\prime}\right)\right] / L,
\end{array}\right. \\
& L=l\left(m^{\prime} n^{\prime \prime}-m^{\prime \prime} n^{\prime}\right)+m\left(n^{\prime} l^{\prime \prime}-n^{\prime \prime} l^{\prime}\right)+n\left(l^{\prime} m^{\prime \prime}-l^{\prime \prime} m^{\prime}\right) .
\end{aligned}
$$

These formulas can be easily obtained by solving a system of three linear equations :

$$
\left\{\begin{array}{l}
l \alpha_{j}+l^{\prime} \beta_{j}+l^{\prime \prime} \gamma_{j}=p_{j}, \\
m \alpha_{j}+m^{\prime} \beta_{j}+m^{\prime \prime} \gamma_{j}=q_{j}, \\
n \alpha_{j}+n^{\prime} \beta_{j}+n^{\prime \prime} \gamma_{j}=r_{j} .
\end{array}\right.
$$

Furthermore, by a simple calculation, we can verify the following:

$$
\begin{aligned}
& \frac{n^{\prime \prime} m^{\prime}-n^{\prime} m^{\prime \prime}}{A}=\frac{l^{\prime \prime} n^{\prime}-l^{\prime} n^{\prime \prime}}{B}=\frac{m^{\prime \prime} l^{\prime}-m^{\prime} l^{\prime \prime}}{C}=\Delta, \\
& \frac{n m^{\prime \prime}-n^{\prime \prime} m}{A^{\prime}}=\frac{l n^{\prime \prime}-l^{\prime \prime} n}{B^{\prime}}=\frac{m l^{\prime \prime}-m^{\prime \prime} l}{C^{\prime}}=\Delta, \\
& \frac{n^{\prime} m-n m^{\prime}}{A^{\prime \prime}}=\frac{l^{\prime} n-l n^{\prime}}{B^{\prime \prime}}=\frac{m^{\prime} l-m l^{\prime}}{C^{\prime \prime}}=\Delta .
\end{aligned}
$$


Substituting these expressions into the right-hand members of (4.8) and (4.9), we get

$$
\alpha_{j}=\frac{\lambda(j)}{\Delta}, \quad \beta_{j}=\frac{\lambda^{\prime}(j)}{\Delta}, \quad \gamma_{j}=\frac{\lambda^{\prime \prime}(j)}{\Delta}, \quad L=\Delta^{2},
$$

which, by virture of (4.4), (4.6), (4.6)', (4.6)" , yields :

$$
\alpha_{j} \geqq 0, \quad \beta_{j} \geqq 0, \quad \gamma_{j} \geqq 0 .
$$

Hence, from the formula (4.7), we see immediately that the expression

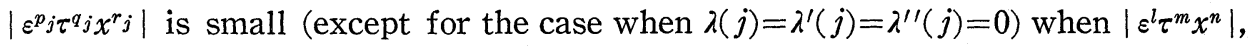
$\left|\varepsilon^{l^{\prime}} \tau^{m^{\prime}} x^{n^{\prime}}\right|$ and $\left|\varepsilon^{l^{\prime \prime}} \tau^{m^{\prime \prime}} x^{n^{\prime \prime}}\right|$ are small or, in other words, $(\varepsilon, \tau, x)$ is in the domain determined by the inequalities :

$$
\left|\varepsilon^{l} \tau^{m} x^{n}\right| \leqq a, \quad\left|\varepsilon^{l^{\prime}} \tau^{m^{\prime}} x^{n^{\prime}}\right| \leqq b, \quad\left|\varepsilon^{l^{\prime \prime}} \tau^{m^{\prime \prime}} x^{n^{\prime \prime}}\right| \leqq c,
$$

where $a, b$ and $c$ are sufficiently small positive constants.

By applying the formula (4.7) to each summand in the formal series (3.1), we have

$$
f \sim \sum_{j=0}^{\infty}\left(\varepsilon^{l} \tau^{m} x^{n}\right)^{\lambda(j) / \Delta}\left(\varepsilon^{l^{\prime}} \tau^{m^{\prime}} x^{n^{\prime}}\right)^{\lambda^{\prime}(j) / \Delta}\left(\varepsilon^{l^{\prime \prime}} \tau^{m^{\prime \prime}} x^{n^{\prime \prime}}\right)^{\lambda^{\prime \prime}(j) / \Delta} f_{j}
$$

\section{$\S 5$. Construction of a formal series.}

Now we try to rearrange the formal series (4.11) in powers of $\left(\varepsilon^{l} \tau^{m} x^{n}\right)$, for example. To do this, we draw a plane parallel to the plane (4.1) through each $\left(p_{j}, q_{j}, r_{j}\right)$. Clearly, this plane is represented by

$$
\lambda(j)=A X+B Y+C Z
$$

and we notice at once that

1) $\left(p_{j}, q_{j}, r_{j}\right)$ and $\left(p_{k}, q_{k}, r_{k}\right)$ lie on a same plane if and only if $\lambda(j)=\lambda(k)$;

2) $\lambda(j)$ is proportional to the distance from the origin to this plane.

From the denumerably infinite set of values $\{\lambda(j)\}$, we pick up all distinct values and arrange them in increasing order. We denote by $\left\{\lambda_{p}\right\}$ the increasing sequence thus obtained.

Next, for each $\lambda_{\rho}$, we consider the totality of the system of the values of $\lambda^{\prime}(j)$ and $\lambda^{\prime \prime}(j)$ such that

$$
\lambda(j)=\lambda_{\rho} \quad \text { (See Fig. 2). }
$$

To those values correspond the denumerably infinite or finite set of points $\left(p_{j}, q_{j}, r_{j}\right)$ which lie on a same plane parallel to (4.1). Obviously, if the set of the points $\left(p_{k}, q_{k}, r_{k}\right)$ is contained in a trigonal pyramid which lies in the interior of the considered pyramid, this will be reduced to the finite set.

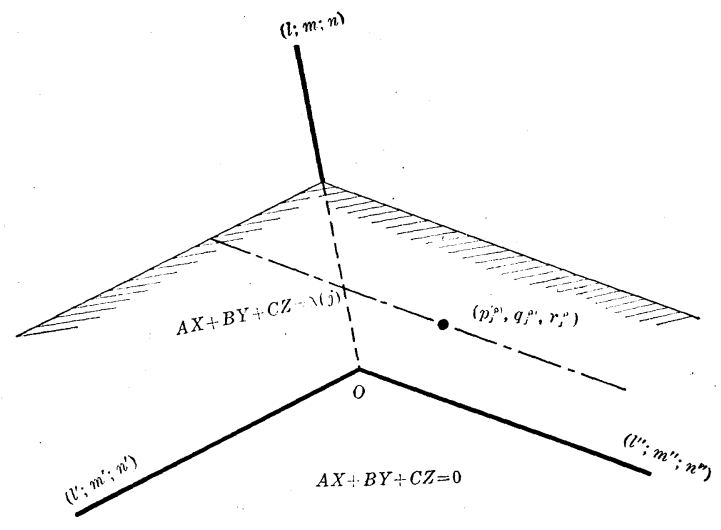

Fig. 2 
We denote by $\left(p_{j}^{(\rho)}, q_{j}^{(\rho)}, r_{j}^{(\rho)}\right)$ the points belonging to this set.

Now we draw a straight line with the directional parameters $\left(l^{\prime \prime} ; m^{\prime \prime} ; n^{\prime \prime}\right)$, for example, through each point $\left(p_{j}^{(\rho)}, q_{j}^{(\rho)}, r_{j}^{(\rho)}\right)$ (See Fig. 2).

Then, by the same reasoning as above, from the denumerably infinite or finite set of values $\left\{\lambda^{\prime}(j)\right\}$ such that $\lambda(j)=\lambda_{\rho}$, we can pick up all distinct values and arrange them in increasing order. Let $\left\{\lambda_{\rho, s}^{\prime}\right\}$ be the increasing sequence thus obtained.

Finally, for each $\lambda_{\rho, 8}^{\prime}$ we consider the totality of the values of $\lambda^{\prime \prime}(j)$ such that

$$
\lambda(j)=\lambda_{\rho}, \quad \lambda^{\prime}(j)=\lambda_{\rho, s}^{\prime}
$$

and arrange them in increasing order. Let $\left\{\lambda_{\rho, s, t}^{\prime \prime}\right\}\left(\lambda_{\rho, s, 1}^{\prime \prime}<\lambda_{\rho, s, 2}^{\prime \prime}<\cdots\right)$ be the sequnce thus obtained.

As we have determined the increasing sequences $\left\{\lambda_{\rho}\right\},\left\{\lambda_{\rho, s}^{\prime}\right\},\left\{\lambda_{\rho, s, t}^{\prime \prime}\right\}$, we can now easily construct a formal series. Firstly, we rearrange the formal series (4.11) in powers of $\left(\varepsilon^{l} \tau^{m} x^{n}\right)$ :

$$
f \sim \sum_{\rho=1}^{\infty}\left(\varepsilon^{l} \tau^{m} x^{n}\right)^{\lambda_{\rho} / \Delta} f_{\rho}\left(\varepsilon^{l^{\prime}} \tau^{m^{\prime}} x^{n^{\prime}}, \varepsilon^{l^{\prime \prime}} \tau^{m^{\prime \prime}} x^{n^{\prime \prime}}\right),
$$

where

$$
f_{\rho}(\xi, \eta) \sim \sum_{j} \xi^{\lambda^{\prime}(j) / \Delta} \eta^{\lambda^{\prime \prime}(j) / \Delta} f_{j} .
$$

The summation is to be taken over all of the indices $j$ such that $\lambda(j)=\lambda_{p}$.

Secondly, we rearrange the formal series $f_{\rho}(\xi, \eta)$ in powers of $\xi$ :

$$
f_{\rho}(\xi, \eta) \sim \sum_{s=1}^{\infty} \xi^{\lambda_{\rho}^{\prime}, s} / \Delta f_{\rho, s}(\eta)
$$

and

$$
f_{\rho, s}(\eta) \sim \sum_{j} \eta^{\lambda^{\prime \prime}(j) / \Delta} f_{j},
$$

where the last summation is to be taken over all of the indices $j$ such that $\lambda(j)=\lambda_{\rho}$ and $\lambda^{\prime}(j)=\lambda_{\rho, 8}^{\prime}$.

Finally, the last formal power series can be written as

$$
f_{\rho, s}(\eta) \sim \sum_{t=1}^{\infty} \eta_{\rho, s, t}^{\lambda_{\rho}^{\prime \prime}} g_{t},
$$

where $g_{t}$ is equal to $f_{j}$ corresponding to an index $j$ such that

$$
\lambda(j)=\lambda_{\rho}, \quad \lambda^{\prime}(j)=\lambda_{\rho, s}^{\prime}, \quad \lambda^{\prime \prime}(j)=\lambda_{\rho, s, t}^{\prime \prime} .
$$

\section{§6. Analytical meaning.}

In a little more general way, we consider one of the following three formal series:

$$
\begin{aligned}
& f \sim \sum_{\rho=1}^{\infty}\left(\varepsilon^{l} \tau^{m} x^{n}\right) \mu_{\rho} f_{\rho}\left(\varepsilon^{l^{\prime}} \tau^{m^{\prime}} x^{n^{\prime}}, \varepsilon^{l \prime \prime} \tau^{m^{\prime \prime}} x^{n^{\prime \prime}}\right), \\
& f \sim \sum_{\rho=1}^{\infty}\left(\varepsilon^{l^{\prime}} \tau^{m^{\prime}} x^{n^{\prime}}\right) \mu_{\rho} f_{\rho}\left(\varepsilon^{l \prime \prime} \tau^{m^{\prime \prime}} x^{n^{\prime \prime}}, \varepsilon^{l} \tau^{m} x^{n}\right), \\
& f \sim \sum_{\rho=1}^{\infty}\left(\varepsilon^{l^{\prime \prime}} \tau^{m^{\prime \prime}} x^{n^{\prime \prime}}\right) \mu_{\rho} f_{\rho}\left(\varepsilon^{l} \tau^{m} x^{n}, \varepsilon^{l^{\prime}} \tau^{m^{\prime}} x^{n^{\prime}}\right),
\end{aligned}
$$


where $f_{\rho}(\xi, \eta)$ are formally expanded in one of the following two formal power series :

$$
\begin{aligned}
& f_{\rho}(\xi, \eta) \sim \sum_{s=1}^{\infty} \xi^{\mu \prime} f_{\rho, s}(\eta) \\
& f_{\rho}(\xi, \eta) \sim \sum_{s=1}^{\infty} \eta^{\mu_{s}^{\prime}} f_{\rho, s}(\xi) .
\end{aligned}
$$

Further $f_{\rho, s}(\zeta)$ admits a formal expansion

$$
f_{\rho, s}(\zeta) \sim \sum_{t=1}^{\infty} \zeta_{t}^{\mu_{t}^{\prime \prime}} f_{\rho, s, t}
$$

$f_{\rho, s, t}$ being constants.

Here, $\left\{\mu_{\rho}\right\},\left\{\mu_{s}^{\prime}\right\}$ and $\left\{\mu_{t}^{\prime \prime}\right\}$ denote increasing sequences, and $(l ; m ; n),\left(l^{\prime} ; m^{\prime} ; n^{\prime}\right)$ and $\left(l^{\prime \prime} ; m^{\prime \prime} ; n^{\prime \prime}\right)$ are directional parameters of the three edges of a trigonal pyramid with its vertex at the origin and containing the interior of the first octant of $(X, Y, Z)$-space. domain

Let $f_{\rho}\left(\eta_{1}, \zeta_{1}\right)$ be functions holomorphic and bounded in $\left(\eta_{1}, \zeta_{1}\right)$ in an angular

$$
\eta_{1} \in \mathfrak{D}\left(\theta_{ \pm}^{\prime} ; d^{\prime}\right), \quad \zeta_{1} \in \mathfrak{D}\left(\theta_{ \pm}^{\prime \prime} ; d^{\prime \prime}\right)^{2)}
$$

and, moreover, admitting a uniform asymptotic expansion in a form of (6.2) (where we have to put $\xi=\eta_{1}, \eta=\zeta_{1}$ ) as $\eta_{1}$ and $\zeta_{1}$ tend to 0 .

We suppose that, for arbitrarily given quantities $\Theta_{-}, \Theta_{+}, \Theta_{-}^{\prime}, \Theta_{+}^{\prime}, \Theta_{-}^{\prime \prime}$ and $\Theta_{+}^{\prime \prime}$, we have

$$
\left\{\begin{array}{l}
\theta_{-}^{\prime}<\arg \left(\varepsilon^{\prime \prime} \tau^{m^{\prime}} x^{n^{\prime}}\right)<\theta_{+}^{\prime}, \\
\theta_{-}^{\prime \prime}<\arg \left(\varepsilon^{\prime \prime \prime} \tau^{m^{\prime \prime}} x^{n^{\prime \prime}}\right)<\theta_{+}^{\prime \prime}
\end{array}\right.
$$

for

$$
\Theta_{-}<\arg \varepsilon<\Theta_{+}, \quad \Theta_{-}^{\prime}<\arg \tau<\Theta_{+}^{\prime}, \quad \Theta_{-}^{\prime \prime}<\arg x<\Theta_{+}^{\prime \prime} .
$$

Then, the function $f(\varepsilon, \tau, x)$ which is holomorphic and bounded in $(\varepsilon, \tau, x)$ in a subdomain

$$
\left\{\begin{array}{lll}
\left|\varepsilon^{l} \tau^{m} x^{n}\right|<a_{1}, & \left|\varepsilon^{\prime \prime} \tau^{m^{\prime}} x^{n^{\prime}}\right|<b_{1}, & \left|\varepsilon^{l \prime \prime} \tau^{m^{\prime \prime}} x^{n^{\prime \prime}}\right|<c_{1}, \\
\varepsilon \in \mathfrak{D}\left(\Theta_{ \pm} ; a_{0}\right), & \tau \in \mathfrak{D}\left(\Theta_{ \pm}^{\prime} ; b_{0}\right), & x \in \mathfrak{D}\left(\Theta_{ \pm}^{\prime \prime} ; c_{0}\right)
\end{array}\right.
$$

will be called asymptotically developable in a form of (6.1) as $\varepsilon$ and $\tau$ tend to 0 in the domain (6.7), if the following conditions are satisfied: for every integer $N>0$, the inequality

$$
\left|f(\varepsilon, \tau, x)-\sum_{\rho=1}^{N-1}\left(\varepsilon^{l} \tau^{m} x^{n}\right) \mu_{\rho} f_{\rho}\left(\varepsilon^{l^{\prime}} \tau^{m^{\prime}} x^{n^{\prime}}, \varepsilon^{l \prime \prime} \tau^{m^{\prime \prime}} x^{n^{\prime \prime}}\right)\right| \leqq K_{N}\left|\varepsilon^{l} \tau^{m} x^{n}\right|^{\mu_{N}}
$$

holds uniformly in the domain (6.7), $K_{N}$ being a certain positive constant independent of $(\varepsilon, \tau, x)$. Here $a_{0}, b_{0}, c_{0}, a_{1}, b_{1}, c_{1}$ are sufficiently small positive constants satisfying the inequalities

$$
\left\{\begin{array}{l}
a_{0}^{l} b_{0}^{m} c_{0}^{n} \leqq a_{1} \\
a_{0}^{l^{\prime}} b_{0}^{m^{\prime}} c_{0}^{n^{\prime}} \leqq b_{1} \leqq d^{\prime} \\
a_{0}^{l^{\prime \prime}} b_{0}^{m^{\prime \prime}} c_{0}^{n^{\prime \prime}} \leqq c_{1} \leqq d^{\prime \prime}
\end{array}\right.
$$

2) For example, $\mathscr{D}\left(\theta_{ \pm}^{\prime} ; d^{\prime}\right)$ denotes an angular region :

$$
\theta_{-}^{\prime}<\arg \eta_{1}<\theta_{+}^{\prime}, \quad 0<\left|\eta_{1}\right|<d^{\prime} .
$$




\section{§ 7. Existence theorem.}

We consider a formal series of the form

$$
f \sim \sum_{t=1}^{\infty} \sum_{s=1}^{\infty} \sum_{\rho=1}^{\infty} \lambda^{\mu_{\rho}} \eta^{\mu_{s}^{\prime} \zeta^{\mu^{\prime}} f_{\rho, s, t}},
$$

where $f_{\rho, s, t}$ are constants $;\left\{\mu_{\rho}\right\},\left\{\mu_{s}^{\prime}\right\}$ and $\left\{\mu_{t}^{\prime \prime}\right\}$ are increasing sequences.

Let

$$
\left\{\begin{array}{lll}
\left|\varepsilon^{l} \tau^{m} x^{n}\right|<a_{1}, & \left|\varepsilon^{l^{\prime}} \tau^{m^{\prime}} x^{n^{\prime}}\right|<b_{1}, & \left|\varepsilon^{l^{\prime \prime}} \tau^{m^{\prime \prime}} x^{n^{\prime \prime}}\right|<c_{1} \\
\varepsilon \in \mathfrak{D}\left(\Theta_{ \pm} ; a_{0}\right), & \tau \in \mathfrak{D}\left(\Theta_{ \pm}^{\prime} ; b_{0}\right), & x \in \mathfrak{D}\left(\Theta_{ \pm}^{\prime \prime} ; c_{0}\right)
\end{array}\right.
$$

be an arbitrarily given subdomain in $(\varepsilon, \tau, x)$-space. Here we suppose that $\Theta_{-}<\Theta_{+}, \Theta_{-}^{\prime}<\Theta_{+}^{\prime}, \Theta_{-}^{\prime \prime}<\Theta_{+}^{\prime \prime} ; a_{0}, b_{0}, c_{0}, a_{1}, b_{1}, c_{1}$ are sufficiently small positive constants $;(l ; m ; n),\left(l^{\prime} ; m^{\prime} ; n^{\prime}\right)$ and $\left(l^{\prime \prime} ; m^{\prime \prime} ; n^{\prime \prime}\right)$ denote the directional parameters of a trigonal pyramid with the same properties as before.

Let

$$
\eta \in \mathfrak{D}\left(\theta_{ \pm}^{\prime} ; d^{\prime}\right), \quad \zeta \in \mathfrak{D}\left(\theta_{ \pm}^{\prime \prime} ; d^{\prime \prime}\right)
$$

be an arbitrarily given subdomain in $(\eta, \zeta)$-space and suppose that the point $\left(\eta^{0}, \zeta^{0}\right)$ defined by $\eta^{0}=\varepsilon_{0}^{l^{\prime}} \tau_{0}^{m^{\prime}} x_{0}^{n^{\prime}}, \zeta^{0}=\varepsilon_{0}^{l^{\prime \prime}} \tau_{0}^{m^{\prime \prime}} x_{0}^{n^{\prime \prime}}$ is contained in (7.3) for $\left(\varepsilon_{0}, \tau_{0}, x_{0}\right)$ in (7.2).

Then we can prove the following proposition:

Proposition. There exists a function $f(\varepsilon, \tau, x)$ holomorphic and bounded in $(\varepsilon, \tau, x)$ in (7.2) and, moreover, admitting a uniform asymptotic expansion in a form, for example,

$$
f(\varepsilon, \tau, x) \sim \sum_{\rho=1}^{\infty}\left(\varepsilon^{l} \tau^{m} x^{n}\right)^{\mu_{\rho}} f_{\rho}\left(\varepsilon^{l^{\prime}} \tau^{m^{\prime}} x^{n^{\prime}}, \varepsilon^{l^{\prime \prime}} \tau^{m^{\prime \prime}} x^{n^{\prime \prime}}\right)
$$

as $\varepsilon$ and $\tau$ tend to 0 in the domain (7.2). The coefficients $f_{\rho}(\eta, \zeta)$ are functions holomorphic, bounded in $(\eta, \zeta)$ and admitting uniform asymptotic expansions in a form, for example,

$$
f_{\rho}(\eta, \zeta) \sim \sum_{s=1}^{\infty} \eta^{\mu \prime} f_{\rho, s}(\zeta)
$$

as $\eta$ tends to 0 in the domain (7.3), where $f_{\rho, s}(\zeta)$ are functions holomorphic, bounded and asymptotically developable in a form

$$
f_{\rho, s}(\zeta) \sim \sum_{t=1}^{\infty} \zeta^{\mu_{t}^{\prime}} f_{\rho, s, t}
$$

as $\zeta$ tends to 0 in the domain $\mathfrak{D}\left(\theta_{ \pm}^{\prime \prime} ; d^{\prime \prime}\right)$.

Indeed, let $\mathfrak{D}\left(\theta_{ \pm} ; d\right)$ be an arbitrary angular domain such that $\lambda^{0}=\varepsilon_{0}^{l} \tau_{0}^{m} x_{0}^{n} \in$ $\mathfrak{D}\left(\theta_{ \pm} ; d\right)$ when $\left(\varepsilon_{0}, \tau_{0}, x_{0}\right)$ is contained in (7.2). Then, it is well known that there exists a function $g(\lambda, \eta, \zeta)$ which is holomorphic and bounded in $(\lambda, \eta, \zeta)$ for

$$
\lambda \in \mathfrak{D}\left(\theta_{ \pm} ; d\right), \quad \eta \in \mathfrak{D}\left(\theta_{ \pm}^{\prime} ; d^{\prime}\right), \quad \zeta \in \mathfrak{D}\left(\theta_{ \pm}^{\prime \prime} ; d^{\prime \prime}\right)
$$

and, moreover, admits a uniform asymptotic expansion in a form

$$
g(\lambda, \eta, \zeta) \sim \sum_{\rho=1}^{\infty} \lambda^{\mu_{\rho}} f_{\rho}(\eta, \zeta)
$$


as $\lambda$ tends to 0 in (7.7), where $f_{\rho}(\eta, \zeta)$ are functions possessing the properties stated in the proposition.

Then, to obtain the proposition, it is sufficient to define the function $f(\varepsilon, \tau, x)$ by

$$
f(\varepsilon, \tau, x) \equiv g\left(\varepsilon^{l} \tau^{m} x^{n}, \varepsilon^{\prime \prime} \tau^{m^{\prime}} x^{n^{\prime}}, \varepsilon^{l^{\prime \prime}} \tau^{m^{\prime \prime}} x^{n^{\prime \prime}}\right) \text {. }
$$

\section{Chapter II. Characteristic polyhedron and division of the domain.}

\section{§ 8. Characteristic polyhedron.}

\section{$1^{\circ}$. Characteristic polyhedron of a system of $n$ equations.}

Let there be given a system of the form

$$
\varepsilon^{\sigma} \tau^{\sigma \prime} \frac{d \vec{y}}{d x}=A(\varepsilon, \tau, x) \vec{y},
$$

where $\sigma$ and $\sigma^{\prime}$ are rational numbers; $A$ is an $n$ by $n$ matrix function of the form:

$$
A(\varepsilon, \tau, x)=\left(\begin{array}{cccccc}
0 & 1 & 0 & \cdots & \cdots & 0 \\
0 & 0 & 1 & 0 & \cdots & \cdots \\
\cdots & \cdots & \cdots & \cdots & \cdots & \cdots \\
0 & 0 & \cdots & \cdots & 0 & 1 \\
a_{n}(\varepsilon, \tau, x) & a_{n-1}(\varepsilon, \tau, x) & \cdots & \cdots & a_{2}(\varepsilon, \tau, x) & 0
\end{array}\right) .
$$

We suppose that

1) $a_{l}(\varepsilon, \tau, x)$ has the following form

$$
a_{l}(\varepsilon, \tau, x)=x^{-m} \imath b_{l}(\varepsilon, \tau, x),
$$

where $m_{l}$ is a nonnegative integer such that, if $m_{l}>0, b_{l}(\varepsilon, \tau, 0) \not \equiv 0$;

2) $b_{l}(\varepsilon, \tau, x)$ is a function holomorphic and bounded in $(\varepsilon, \tau, x)$ for

$$
|\varepsilon|<a_{0}, \quad|\tau|<b_{0}, \quad|x|<c_{0} ;
$$

$3)$ there exists at least one index $l$ such that $b_{l}(0,0, x) \not 0$.

Then, from the condition 2$)$ we see that the functions $a_{l}(\varepsilon, \tau, x)$ admit Laurent expansion in uniformly convergent triple power series of $\varepsilon, \tau$ and $x$ :

$$
a_{l}(\varepsilon, \tau, x)=\sum_{p} \sum_{q} \sum_{r} \varepsilon^{p} \tau^{q} x^{r} a_{l}^{(p, q, r)}
$$

for

$$
|\varepsilon|<a_{0}, \quad|\tau|<b_{0}, \quad 0<|x|<c_{0},
$$

where $a_{l}^{(p, q, r)}$ are constants.

We consider $(X, Y, Z)$-space whose coordinates are represented by $(X, Y, Z)$ and plot the following points:

$$
\left\{\begin{array}{l}
\mathrm{R}=\left(\sigma, \sigma^{\prime},-1\right), \\
\mathrm{P}_{l}^{(p, q, r)}=\left(\frac{p}{l}, \frac{q}{l}, \frac{r}{l}\right)
\end{array}\right.
$$

for $a_{l}^{(p, q, r)} \neq 0$.

Denote by $I I$ a polyhedron, convex downwards, such that its vertices are some of the points (8.3) and none of the points (8.3) is located outside $\Pi$, We 
will call it a characteristic polyhedron of the system (8.1).

In case when all of the functions $a_{l}(\varepsilon, \tau, x)$ are holomorphic at $x=0$ (this means that $\left.m_{l}=0\right)$, the points (8.3) except for the point $\mathrm{R}$ do not lie below $(X, Y)$-plane. Let $\Pi^{\prime}$ be the polyhedron cut off from the characteristic polyhedron $\Pi$ by $(X, Y)$-plane. Then, $\Pi^{\prime}$ will be said to be the modified characteristic polyhedron of the system (8.1). Consequently, the vertices of the modified polyhedron consist of those of the polyhedron $I I$ and of the vertices of the polygonal line at which the sides of $I$ intersect with $(X, Y)$-plane.

Let $\mathrm{V}$ be any one of the vertices of $\Pi$ or $\Pi^{\prime}$. Clearly, a finite number of sides meet at the vertex $\mathrm{V}$, say $\mathrm{S}_{1}, \cdots, \mathrm{S}_{m}(m \geqq 3)$. Consider any three of such sides, say $\mathrm{S}_{r}, \mathrm{~S}_{s}, \mathrm{~S}_{t}$ and the three planes $\widetilde{\mathrm{S}}_{r}, \widetilde{\mathrm{S}}_{s}, \widetilde{\mathrm{S}}_{t}$ which contain respectively the three sides $\mathrm{S}_{r}, \mathrm{~S}_{s}, \mathrm{~S}_{t}$. Then, it is easily seen that those three planes form a trigonal pyramid $\mathrm{S}_{r} \cup \mathrm{S}_{s} \cup \mathrm{S}_{t}$ such that its vertex is the point $\mathrm{V}$ and it encloses the polyhedron $\Pi$ or $\Pi^{\prime}$. Such a trigonal pyramid will be called a characteristic trigonal pyramid at $\mathrm{V}$. Clearly, there exist $\left(\begin{array}{c}m \\ 3\end{array}\right)$ characteristic trigonal pyramids at the vertex $\mathrm{V}$.

\section{$2^{\circ}$. Application to Whittaker's differential equation.}

We consider the differential equation ( $\mathfrak{B})$ :

$$
\varepsilon^{\frac{1}{2} \tau} \frac{d \vec{y}}{d x}=\left(\begin{array}{cc}
0 & 1 \\
\varepsilon x^{-2}-\tau^{2} x^{-3}+\frac{1}{4} \varepsilon \tau^{2} x^{-4}\left(1-x^{2}\right) & 0
\end{array}\right) \vec{y} .
$$

To construct a characteristic polyhedron of the system (W), it is convenient to consider the following differential equation (with a form a little more general than (W)):

$$
\varepsilon^{\frac{1}{2} \tau} \frac{d \vec{y}}{d x}=\left(\begin{array}{cc}
0 & 1 \\
\varepsilon x^{-2} f_{10}-\tau^{2} x^{-3} f_{02}+\frac{1}{4} \varepsilon \tau^{2} x^{-4} f_{12} & 0
\end{array}\right) \vec{y},
$$

where $f_{j k} \equiv f_{j k}(\varepsilon, \tau, x)$ are functions holomorphic and bounded in $(\varepsilon, \tau, x)$ for

$$
|\varepsilon|<a_{0}, \quad|\tau|<b_{0}, \quad|x|<c_{0}
$$

and developable in uniformly convergent triple power series of $\varepsilon, \tau, x$ :

$$
f_{j k}(\varepsilon, \tau, x)=1+\sum_{r} \sum_{q} \sum_{p} \varepsilon^{p} \tau^{q} x^{r} f_{j k}^{(p, q, r)} \quad(p+q+r \geqq 1) .
$$

Then, we notice that the modified system $(\widetilde{\mathfrak{B}})$ has the same form as (8.1) and the points (8.3) become:

$$
\left\{\begin{array}{l}
\left\{\begin{array}{l}
\mathrm{P}_{2}^{(1,0,-2)}=\left(\frac{1}{2}, 0,-1\right), \\
\mathrm{P}_{2}^{(1+p, q, r-2)}=\left(\frac{1}{2}+\frac{p}{2}, \frac{q}{2},-1+\frac{r}{2}\right) ;
\end{array}\right. \\
\left\{\begin{array}{l}
\mathrm{P}_{2}^{(0,2,-3)}=\left(0,1,-\frac{3}{2}\right), \\
\mathrm{P}_{2}^{(p, 2+q, r-3)}=\left(\frac{p}{2}, 1+\frac{q}{2},-\frac{3}{2}+\frac{r}{2}\right) ;
\end{array}\right.
\end{array}\right.
$$




$$
\left\{\begin{array}{l}
\mathrm{P}_{2}^{(1,2,-4)}=\left(\frac{1}{2}, 1,-2\right), \\
\mathrm{P}_{2}^{(1+p, 2+q, r-4)}=\left(\frac{1}{2}+\frac{p}{2}, 1+\frac{q}{2},-2+\frac{r}{2}\right) \\
\mathrm{R} \quad=\left(\frac{1}{2}, 1,-1\right) .
\end{array}\right.
$$

Therefore, as is shown in figure, a characteristic polyhedron consists of three vertices:

$$
\begin{aligned}
& \mathrm{V}_{1}=\mathrm{P}_{2}^{(1,2,-4)}=\left(\frac{1}{2}, 1,-2\right) ; \\
& \mathrm{V}_{2}=\mathrm{P}_{2}^{(0,2,-3)}=\left(0,1,-\frac{3}{2}\right) ; \\
& \mathrm{V}_{3}=\mathrm{P}_{2}^{(1,0,-2)}=\left(\frac{1}{2}, 0,-1\right) ;
\end{aligned}
$$

nine edges and seven sides-three sides are parallel to $(X, Y),(Y, Z),(Z, X)$-plane respectively and three sides are perpendicular to the coordinate planes respectively (See Fig. 3).

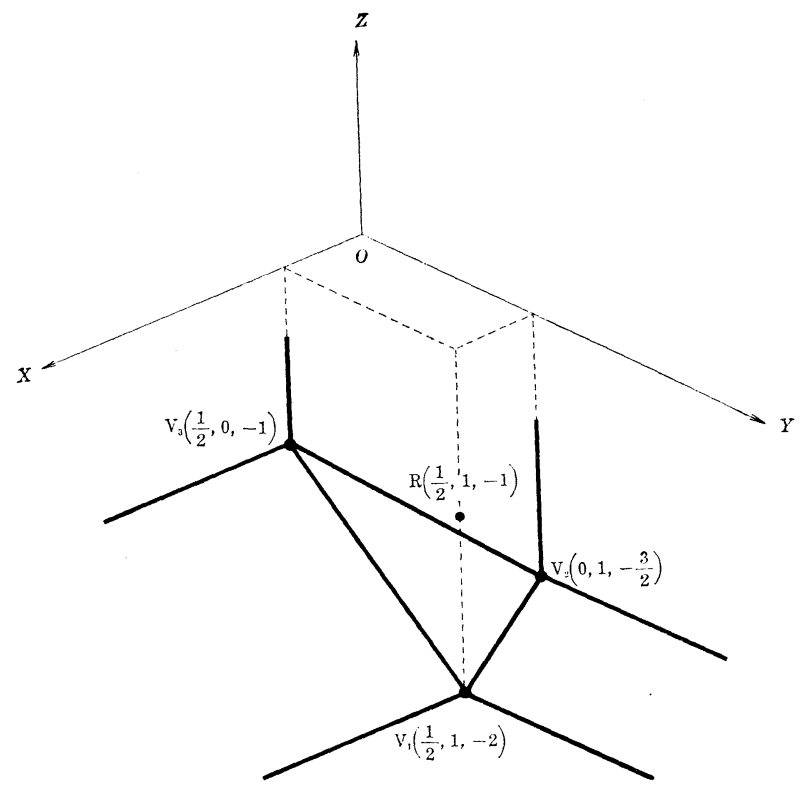

Fig. 3

\section{§. Characteristic trigonal pyramids.}

We have already seen in $\S 8$ that the characteristic polyhedron consists of three vertices:

$$
\mathrm{V}_{1}=\left(\frac{1}{2}, 1,-2\right), \quad \mathrm{V}_{2}=\left(0,1,-\frac{3}{2}\right), \quad \mathrm{V}_{3}=\left(\frac{1}{2}, 0,-1\right) .
$$

Since at each vertex four sides meet, there exist four characteristic trigonal 
pyramids. Thus, we have in all twelve characteristic trigonal pyramids.

Now we will calculate the directional parameters of the three edges of such pyramids.

$1^{\circ}$. Characteristic trigonal pyramids at the vertex $V_{1}$.

If we change the coordinate system from $(X, Y, Z)$ to $\left(X^{\prime}, Y^{\prime}, Z^{\prime}\right)$ :

$$
X^{\prime}=X-\frac{1}{2}, \quad Y^{\prime}=Y-1, \quad Z^{\prime}=Z+2,
$$

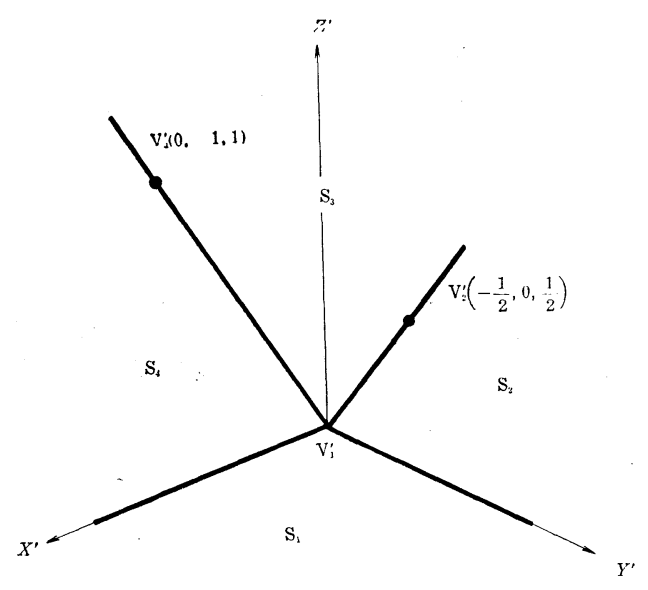

Fig. 4 the vertex $V_{1}$ is at the origin. The four sides which meet at the vertex $\mathrm{V}_{1}$ are expressed by

$$
\begin{array}{rlrl}
\mathrm{S}_{1}: & & Z^{\prime} & =0 ; \\
\mathrm{S}_{2}: & X^{\prime} & +Z^{\prime} & =0 ; \\
\mathrm{S}_{3}: & X^{\prime}+Y^{\prime}+Z^{\prime} & =0 ; \\
\mathrm{S}_{4}: & Y^{\prime}+Z^{\prime} & =0
\end{array}
$$

(See Fig 4).

From these equations we can easily calculate (using the formular (4.5), (4.5)', $\left.(4.5)^{\prime \prime}\right)$ the directional parameters of the three edges of four trigonal pyramids at the vertex $\mathrm{V}_{1}$ and we get a following table:

$$
\left\{\begin{array}{lll}
(1 ;-1 ; 0), \quad(0 ; 1 ; 0), \quad(-1 ; 0 ; 1), & S_{3} \cup S_{1} \cup S_{2} \\
(1 ; 0 ; 0), \quad(0 ; 1 ; 0), \quad(-1 ;-1 ; 1), & S_{4} \cup S_{1} \cup S_{2} \\
(1 ; 0 ; 0), \quad(-1 ; 1 ; 0), \quad(0 ;-1 ; 1), & S_{4} \cup S_{1} \cup S_{3} ; \\
(1 ; 1 ;-1), \quad(-1 ; 0 ; 1), \quad(0 ;-1 ; 1), & S_{4} \cup S_{2} \cup S_{3} .
\end{array}\right.
$$

$2^{\circ}$. Characteristic trigonal pyramids at the vertex $V_{2}$.

We change the coordinate system from $(X, Y, Z)$ to $\left(X^{\prime}, Y^{\prime}, Z^{\prime}\right)$ :

$$
\begin{array}{r}
X^{\prime}=X, \quad Y^{\prime}=Y-1, \\
Z^{\prime}=Z+\frac{3}{2} .
\end{array}
$$

Then, the four sides which meet at the vertex $V_{2}$ are expressed by the following equations (See Fig. 5) :

$$
\begin{aligned}
& \mathrm{S}_{1}: X^{\prime}+Z^{\prime}=0 ; \\
& \mathrm{S}_{2}: \quad X^{\prime}=0 ; \\
& \mathrm{S}_{3}: 2 X^{\prime}+Y^{\prime}=0 ; \\
& \mathrm{S}_{4}: \quad X^{\prime}+Y^{\prime}+Z^{\prime}=0 .
\end{aligned}
$$

There exist four characteristic trigonal pyramids and the directional parameters of the three

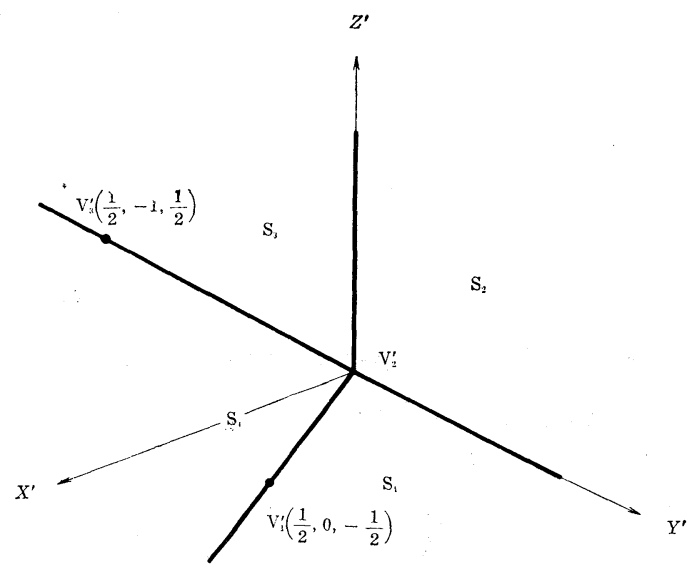

Fig. 5 
edges of such four pyramids are given by a following table:

$$
\left\{\begin{array}{lll}
(1 ;-2 ;-1),(0 ; 1 ; 0),(0 ; 0 ; 1), & S_{3} \cup S_{1} \cup S_{2} \\
(1 ; 0 ;-1),(0 ; 1 ; 0),(0 ;-1 ; 1), & S_{4} \cup S_{1} \cup S_{2} ; \\
(1 ; 0 ;-1),(-1 ; 2 ; 1),(1 ;-2 ; 1), & S_{4} \cup S_{1} \cup S_{3} ; \\
(0 ; 1 ;-1),(0 ; 0 ; 1),(1 ;-2 ; 1), & S_{4} \cup S_{2} \cup S_{3} .
\end{array}\right.
$$

\section{$3^{\circ}$. Characteristic trigonal pyramids at the vertex $\mathrm{V}_{3}$.}

Since the vertex $\mathrm{V}_{3}$ is located at $\left(\frac{1}{2}, 0,-1\right)$, we change the coordinate system from $(X, Y, Z)$ to $\left(X^{\prime}, Y^{\prime}, Z^{\prime}\right)$ :

$$
X^{\prime}=X-\frac{1}{2}, \quad Y^{\prime}=Y, \quad Z^{\prime}=Z+1 .
$$

Then, as is shown in figure, we have a tetragonal pyramid whose four sides are expressed by the equations (See Fig. 6):



Fig. 6

$$
\begin{aligned}
\mathrm{S}_{1}: & Y^{\prime}+Z^{\prime} & =0 ; \\
\mathrm{S}_{2}: & X^{\prime}+Y^{\prime}+Z^{\prime} & =0 ; \\
\mathrm{S}_{3}: & 2 X^{\prime}+Y^{\prime} & =0 ; \\
\mathrm{S}_{4}: & Y^{\prime} & =0
\end{aligned}
$$

By an elementary calculation we see that the directional parameters of the three edges of four characteristic trigonal pyramids are given by:

$$
\left\{\begin{array}{lll}
(0 ; 1 ;-1),(-1 ; 2 ;-1),(1 ;-2 ; 2), & S_{1} \cup S_{2} \cup S_{3} ; \\
(1 ; 0 ; 0),(0 ; 1 ;-1),(-1 ; 0 ; 1), & S_{4} \cup S_{1} \cup S_{2} ; \\
(1 ; 0 ; 0),(-1 ; 2 ;-2),(0 ; 0 ; 1), & S_{4} \cup S_{1} \cup S_{3} ; \\
(1 ; 0 ;-1),(-1 ; 2 ;-1),(0 ; 0 ; 1), & S_{4} \cup S_{2} \cup S_{3} .
\end{array}\right.
$$


$\S 10$. Division of the domain $|\varepsilon| \leqq a_{0},|\tau| \leqq b_{0},|x| \leqq c_{0}$.

\section{$1^{\circ}$. Principal subdomains.}

According to the tables (9.2), (9.4), (9.6), we consider following twelve domains in $(\varepsilon, \tau, x)$-space :

$$
\begin{aligned}
& \left|\varepsilon \tau^{-1}\right| \leqq a_{1}, \\
& \left|\varepsilon^{-1} x\right| \leqq c_{1} ;{ }^{3)} \\
& \left|\varepsilon^{-1} \tau^{-1} x\right| \leqq c_{2} \text {; } \\
& \left|\varepsilon^{-1} \tau\right| \leqq b_{3}, \quad\left|\tau^{-1} x\right| \leqq c_{3} ; \\
& \left|\varepsilon \tau x^{-1}\right| \leqq a_{4}, \quad\left|\varepsilon^{-1} x\right| \leqq b_{4}, \quad\left|\tau^{-1} x\right| \leqq c_{4} ; \\
& \left|\varepsilon \tau^{-2} x^{-1}\right| \leqq a_{5} \text {, } \\
& \left|\varepsilon x^{-1}\right| \leqq a_{6}, \\
& \left|\tau^{-1} x\right| \leqq c_{6} ; \\
& \left|\varepsilon x^{-1}\right| \leqq a_{7}, \quad\left|\varepsilon^{-1} \tau^{2} x\right| \leqq b_{7}, \quad\left|\varepsilon \tau^{-2} x\right| \leqq c_{7} ; \\
& \left|\tau x^{-1}\right| \leqq a_{8}, \quad\left|\varepsilon \tau^{-2} x\right| \leqq c_{8} \text {; } \\
& \left|\tau x^{-1}\right| \leqq a_{9}, \quad\left|\varepsilon^{-1} \tau^{2} x^{-1}\right| \leqq b_{9}, \quad\left|\varepsilon \tau^{-2} x^{2}\right| \leqq c_{9} ; \\
& \left|\tau x^{-1}\right| \leqq b_{10}, \quad\left|\varepsilon^{-1} x\right| \leqq c_{10} ; \\
& \left|\varepsilon x^{-1}\right| \leqq a_{12}, \quad\left|\varepsilon^{-1} \tau^{2} x^{-1}\right| \leqq b_{12},
\end{aligned}
$$

where $a_{i}, b_{i}, c_{i}$ are sufficiently small positive constants. These are the subdomains of

$$
(\varepsilon, \tau, x) \in\left\{\varepsilon:|\varepsilon| \leqq a_{0}\right\} \times\left\{\tau:|\tau| \leqq b_{0}\right\} \times\left\{x:|x| \leqq c_{0}\right\} .
$$

These subdomains will be called principal subdomains related with the given differential equation.

From the above twelve subdomains let us pick up, for example, the following six subdomains :
$\left(\mathrm{D}_{1}\right)$
$\left|\varepsilon \tau^{-1}\right| \leqq a_{1}$,
$\left(\mathrm{D}_{2}\right)$
$\left(\mathrm{D}_{3}\right)$
$\left|\varepsilon x^{-1}\right| \leqq a_{3}$,
$\left|\varepsilon^{-1} x\right| \leqq c_{1}$
$\left(\mathrm{D}_{4}\right)$
$\left|\tau x^{-1}\right| \leqq a_{4}$,
$\left|\varepsilon^{-1} \tau\right| \leqq b_{2}$,
$\left|\tau^{-1} x\right| \leqq c_{2}$;
$\left|\tau^{-1} x\right| \leqq c_{3}$;
$\left(\mathrm{D}_{5}\right)$
$\left|\tau x^{-1}\right| \leqq a_{5}$,
$\left|\varepsilon \tau^{-2} x\right| \leqq c_{4}$;
$\left(\mathrm{D}_{6}\right)$
$\left|\varepsilon^{-1} \tau^{2} x^{-1}\right| \leqq b_{5}, \quad\left|\varepsilon \tau^{-2} x^{2}\right| \leqq c_{5} ;$
$\left|\varepsilon^{-1} \tau^{2} x^{-2}\right| \leqq b_{6}$,

which correspond respectively to the domains (10.1), (10.3), (10.6), (10.8), (10.9), (10.11).

\section{$2^{\circ}$. Auxiliary subdomains.}

In order to obtain the complete covering of the original domain (10.0),

3) The subdomains $(10.1), \cdots,(10.12)$ are all defined by the inequalities of the form

$$
\left|\varepsilon^{l} \tau^{m} x^{n}\right| \leqq a, \quad\left|\varepsilon^{l \prime} \tau^{m \prime} x^{n \prime}\right| \leqq b, \quad\left|\varepsilon^{l \prime \prime} \tau^{m \prime \prime} x^{n \prime \prime}\right| \leqq c .
$$

If, for example, $m^{\prime}>0, l^{\prime}=n^{\prime}=0$, the second inequality is reduced to

$$
|\tau| \leqq b^{\prime} \text {. }
$$

In such a case, we omit the second inequality for simplicity's sake. Thus (10.1) is an abbreviated form of

$$
\left|\varepsilon \tau^{-1}\right| \leqq a_{1}, \quad|\tau| \leqq b_{1}, \quad\left|\varepsilon^{-1} x\right| \leqq c_{1} .
$$

This convention will be adopted hereafter in this paper. 
principal subdomains are nöt sufficient. So we add following five subdomains :$$
b_{7} \leqq\left|\varepsilon^{-1} \tau\right| \leqq B_{7}
$$

$\left(\mathrm{D}_{8}\right) \quad\left|\varepsilon \tau^{-1}\right| \leqq a_{8}$,

$$
\begin{gathered}
c_{8} \leqq\left|\varepsilon^{-1} x\right| \leqq C_{8} ; \\
c_{9} \leqq\left|\varepsilon \tau^{-2} x^{2}\right| \leqq C_{9} ; \\
c_{10} \leqq\left|\tau^{-1} x\right| \leqq C_{10} ; \\
c_{11} \leqq\left|\varepsilon \tau^{-2} x\right| \leqq C_{11},
\end{gathered}
$$

where $A_{i}, B_{i}, C_{i}$ are sufficiently large positive constants.

These subdomains are called auxiliary subdomains.

\section{$\S 11$. Possibility of covering of the domain (10.0).}

We can assert that the domain (10.0) is covered by these eleven subdomains without lacuna. Indeed, this assertion will be easily verified from the following:

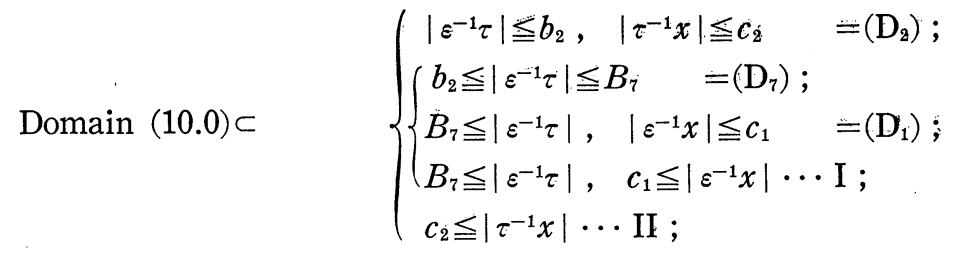

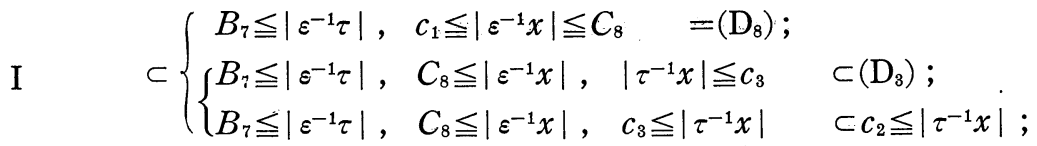

$$
\begin{aligned}
& \text { II } \quad \subset \begin{cases}c_{2} \leqq\left|\tau^{-1} x\right| \leqq C_{10}=\left(\mathrm{D}_{10}\right) ; \\
C_{10} \leqq\left|\tau^{-1} x\right|, & \left|\varepsilon \tau^{-2} x\right| \leqq c_{4} \quad=\left(\mathrm{D}_{4}\right), \\
C_{10} \leqq\left|\tau^{-1} x\right|, & c_{4} \leqq\left|\varepsilon \tau^{-2} x\right| \leqq C_{11} \quad \subset\left(\mathrm{D}_{11}\right), \\
C_{10} \leqq\left|\tau^{-1} x\right|, & C_{11} \leqq\left|\varepsilon \tau^{-2} x\right| \cdots \text { III } ;\end{cases} \\
& \text { III } \quad \subset\left\{\begin{array}{lll}
C_{10} \leqq\left|\tau^{-1} x\right|, & C_{11} \leqq\left|\varepsilon \tau^{-2} x\right|, & \left|\varepsilon \tau^{-2} x^{2}\right| \leqq c_{5} \quad=\left(\mathrm{D}_{5}\right) ; \\
\mathrm{C}_{10} \leqq\left|\tau^{-1} x\right|, & C_{11} \leqq\left|\varepsilon \tau^{-2} x\right|, & c_{5} \leqq\left|\varepsilon \tau^{-2} x^{2}\right| \leqq C_{9} \quad \subset\left(\mathrm{D}_{9}\right) ; \\
C_{10} \leqq\left|\tau^{-1} x\right|, & C_{11} \leqq\left|\varepsilon \tau^{-2} x\right|, & C_{9} \leqq\left|\varepsilon \tau^{-2} x^{2}\right| \quad \subset\left(\mathrm{D}_{6}\right) .
\end{array}\right.
\end{aligned}
$$

As we shall see from this proposition, it seems that the choice of the subdomains such that their union covers without lacuna the given domain is not always unique, while, in the case when the given differential equations depend on one parameter, such a division of the domain was uniquely determined.

\section{Chapter III. Study of the behavior of the solutions in the principal subdomains $\left(\mathbf{D}_{1}\right),\left(\mathbf{D}_{2}\right),\left(\mathbf{D}_{3}\right),\left(\mathbf{D}_{4}\right),\left(\mathbf{D}_{5}\right),\left(\mathbf{D}_{6}\right)$.}

In this chapter, we will show that, in order to investigate the behavior of the solutions in the principal subdomains $\left(D_{i}\right)(i=1,2, \cdots, 6)$, it is sufficient to study following two systems: 


$$
\begin{gathered}
\left(\alpha^{l} \beta^{m} \xi^{n}\right)^{p}\left(\alpha^{l^{\prime}} \beta^{m^{\prime}} \xi^{n^{\prime}}\right)^{p^{\prime}}\left(\alpha^{l^{\prime \prime}} \beta^{m^{\prime \prime}} \xi^{n^{\prime \prime}}\right)^{p^{\prime \prime}} \cdot \xi \frac{d \overrightarrow{\mathfrak{w}}}{d \xi}=A(\alpha, \beta, \xi) \overrightarrow{\mathfrak{w}} \\
\left(p>0, p^{\prime} \geqq 0, p^{\prime \prime} \geqq 0, n p+n^{\prime} p^{\prime}+n^{\prime \prime} p^{\prime \prime} \neq 0\right) ; \\
\left(\alpha^{l} \beta^{m} \xi^{n}\right)^{p}\left(\alpha^{l^{\prime}} \beta^{m^{\prime}} \xi^{n^{\prime}}\right)^{p^{\prime}}\left(\alpha^{l^{\prime \prime}} \beta^{m^{\prime \prime}} \xi^{n^{\prime \prime}}\right)^{p^{\prime \prime}} \cdot \xi \frac{d \overrightarrow{\mathfrak{w}}}{d \xi}=A(\alpha, \beta, \xi) \overrightarrow{\mathfrak{w}} \\
\left(p>0, p^{\prime} \geqq 0, p^{\prime \prime} \geqq 0, n p+n^{\prime} p^{\prime}+n^{\prime \prime} p^{\prime \prime}=0\right),
\end{gathered}
$$

where

(i ) $\left(p, p^{\prime}, p^{\prime \prime}\right)$ are integers;

(ii) $(l ; m ; n),\left(l^{\prime} ; m^{\prime} ; n^{\prime}\right),\left(l^{\prime \prime} ; m^{\prime \prime} ; n^{\prime \prime}\right)$ are rational numbers and denote the directional parameters of the three edges of a trigonal pyramid which encloses the first octant of $(X, Y, Z)$-space;

(iii) $A(\alpha, \beta, \xi)$ is a matrix function with certain properties.

$\S 12$. Transformation of the system $(\mathfrak{B})$ in the domain $\left(D_{1}\right)$.

\section{$\mathbf{1}^{\circ}$. Transformation.}

We should notice that the subdomain $\left(D_{1}\right)$ (or $D_{2}$ ) corresponds to the characteristic trigonal pyramid with the vertex $\mathrm{V}_{1}=\left(\frac{1}{2}, 1,-2\right)$. So let us make a transformation of the form

$$
\vec{y}=\left(\begin{array}{cc}
1 & 0 \\
0 & \varepsilon^{\frac{1}{2}} \tau x^{-2}
\end{array}\right) \vec{z}
$$

Then, the system (W) is changed into a system

$$
x \cdot x \frac{d \vec{z}}{d x}=\left(\begin{array}{cc}
0 & 1 \\
\tau^{-2} x^{2}-\varepsilon^{-1} x+\frac{1}{4}-\frac{1}{4} x^{2} & 2 x
\end{array}\right) \vec{z} .
$$

Since we can write

$$
\left\{\begin{aligned}
x & =\left(\varepsilon \tau^{-1}\right) \tau\left(\varepsilon^{-1} x\right), \\
\tau^{-1} x & =\left(\varepsilon \tau^{-1}\right)\left(\varepsilon^{-1} x\right),
\end{aligned}\right.
$$

we see immediately that the coefficient matrix of (12.2) becomes a matrix function whose components are polynomials of $\left(\varepsilon \tau^{-1}\right), \tau, \varepsilon^{-1} x$. Further, the expressions $\varepsilon \tau^{-1}, \tau, \varepsilon^{-1} x$ being supposed to be sufficiently small in the considered subdomain $\left(\mathrm{D}_{1}\right)$ :

$$
\left|\varepsilon \tau^{-1}\right| \leqq a_{1}, \quad 0<|\tau| \leqq b_{0}, \quad\left|\varepsilon^{-1} x\right| \leqq c_{1},
$$

it is easily found that the coefficient matrix has two distinct characteristic values. And, those values tend to $\pm 1 / 2$ when the quantities $\left|\varepsilon \tau^{-1}\right|,|\tau|,\left|\varepsilon^{-1} x\right|$ tend to zero.

\section{$2^{\circ}$. Reduced form of Type $A$.}

Therefore, in order to study the system (12.2) in the domain $\left(D_{1}\right)$, it is sufficient to consider a system of the following type:

$$
\begin{aligned}
& \left(\alpha^{l} \beta^{m} \xi^{n}\right)^{p}\left(\alpha^{l^{\prime}} \beta^{m^{\prime}} \xi^{n^{\prime}}\right)^{p^{\prime}}\left(\alpha^{l^{\prime \prime}} \beta^{m^{\prime \prime}} \xi^{n^{\prime \prime}}\right)^{p^{\prime \prime}} \cdot \xi \frac{d \overrightarrow{\mathfrak{w}}}{d \xi}=A(\alpha, \beta, \xi) \overrightarrow{\mathfrak{w}} \\
& \left(p>0, p^{\prime} \geqq 0, p^{\prime \prime} \geqq 0, n p+n^{\prime} p^{\prime}+n^{\prime \prime} p^{\prime \prime} \neq 0\right),
\end{aligned}
$$


where we suppose that

1) $A(\alpha, \beta, \xi)$ is a matrix function which is holomorphic and bounded in $(\alpha, \beta, \xi)$ for

$$
\left\{\begin{array}{l}
\left|\alpha^{l} \beta^{m} \xi^{n}\right|<a_{1},\left|\alpha^{l^{\prime}} \beta^{m^{\prime}} \xi^{n^{\prime}}\right|<b_{1},\left|\alpha^{l^{\prime \prime}} \beta^{m^{\prime \prime}} \xi^{n^{\prime \prime}}\right|<c_{1}, \\
\alpha \in \mathfrak{D}\left(\Theta_{ \pm} ; a\right), \beta \in \mathscr{D}\left(\Theta_{ \pm}^{\prime} ; b\right), \xi \in \mathfrak{D}\left(\Theta_{ \pm}^{\prime \prime} ; c\right) .
\end{array}\right.
$$

Moreover, this matrix admits a uniform asymptotic expansion in powers of $\left(\alpha^{l} \beta^{m} \xi^{n}\right)$ :

$$
A(\alpha, \beta, \xi) \sim \sum_{h=0}^{\infty}\left(\alpha^{l} \beta^{m} \xi^{n}\right)^{h} A_{h}\left(\alpha^{l^{\prime}} \beta^{m^{\prime}} \xi^{n^{\prime}}, \alpha^{l^{\prime \prime}} \beta^{m^{\prime \prime} \xi^{n^{\prime \prime}}}\right)
$$

as $\alpha$ and $\beta$ tend to 0 in the domain (12.4);

2) $A_{h}(\eta, \zeta)$ are matrix functions holomorphic and bounded in $(\eta, \zeta)$ for

$$
\eta \in \mathfrak{D}\left(\theta_{ \pm}^{\prime} ; d^{\prime}\right), \quad \zeta \in \mathfrak{D}\left(\theta_{ \pm}^{\prime \prime} ; d^{\prime \prime}\right)
$$

and admitting uniform asymptotic expansions of a form

$$
A_{h}(\eta, \zeta) \sim \sum_{k=0}^{\infty} \eta^{k} A_{h k}(\zeta)
$$

as $\eta$ tends to 0 in the domain (12.5);

3) $A_{h k}(\zeta)$ are matrix functions holomorphic, bounded and asymptotically developable in powers of $\zeta$ :

$$
A_{h k}(\zeta) \sim \sum_{l=0}^{\infty} \zeta^{l} A_{h k l}
$$

as $\zeta$ tends to 0 in the domain

$$
\zeta \in \mathfrak{D}\left(\theta_{ \pm}^{\prime \prime} ; d^{\prime \prime}\right)
$$

where $A_{h k l}$ are constant matrices;

4) the matrix $A_{000}$ has two distinct characteristic values;

5) the domain (12.5) contains always the point $(\eta, \zeta)=\left(\alpha^{l^{\prime}} \beta^{m^{\prime}} \xi^{n^{\prime}}, \alpha^{l^{\prime \prime}} \beta^{m^{\prime \prime}} \xi^{n^{\prime \prime}}\right)$ provided that the point $(\alpha, \beta, \xi)$ belongs to the domain (12.4).

$3^{\circ}$. Thus we have proved the following:

I. In the subdomain

$\left(\mathrm{D}_{1}\right)$

$$
\left|\varepsilon \tau^{-1}\right| \leqq a_{1}, \quad\left|\varepsilon^{-1} x\right| \leqq c_{1},
$$

the system (W) is reduced to a system of Type A by a transformation

$$
\left\{\begin{array}{l}
\vec{y}=\left(\begin{array}{cc}
1 & 0 \\
0 & \varepsilon^{\frac{1}{2}} \tau x^{-2}
\end{array}\right) \overrightarrow{\mathfrak{w}}, \\
\varepsilon=\alpha, \\
\tau=\beta, \\
x=\xi .
\end{array}\right.
$$

§13. Domain $\left(\mathbf{D}_{2}\right)$.

The discussion will be carried out in quite a similar way. We consider again the system (12.2) in the subdomain $\left(\mathrm{D}_{2}\right)$ :

$$
0<|\varepsilon| \leqq a_{0}, \quad\left|\varepsilon^{-1} \tau\right| \leqq b_{2}, \quad\left|\tau^{-1} x\right| \leqq c_{2} .
$$


Since we can write

$$
\left\{\begin{aligned}
x & =\varepsilon\left(\varepsilon^{-1} \tau\right)\left(\tau^{-1} x\right), \\
\varepsilon^{-1} x & =\left(\varepsilon^{-1} \tau\right)\left(\tau^{-1} x\right)
\end{aligned}\right.
$$

it is easily seen that the coefficient matrix of the system (12.2) is regarded as a polynomial of $\varepsilon,\left(\varepsilon^{-1} \tau\right),\left(\tau^{-1} x\right)$ and has two distinct characteristic values in the domain $\left(\mathrm{D}_{2}\right)$.

Hence, we proved the following:

\section{In the subdomain}

$$
\left|\varepsilon^{-1} \tau\right| \leqq b_{2}, \quad\left|\tau^{-1} x\right| \leqq c_{2},
$$

the same transformation as in the domain $\left(\mathrm{D}_{1}\right)$ yields a system of Type $\mathrm{A}$.

§14. Subdomains $\left(\mathbf{D}_{3}\right),\left(\mathbf{D}_{4}\right)$.

We should notice that the domains $\left(D_{3}\right),\left(D_{4}\right)$ correspond to the characteristic trigonal pyramids with the vertex $\mathrm{V}_{2}=\left(0,1,-\frac{3}{2}\right)$. So let us make a transformation

$$
\vec{y}=\left(\begin{array}{cc}
1 & 0 \\
0 & \tau x^{-\frac{3}{2}}
\end{array}\right) \vec{z}
$$

Then, the system (WB) will be transformed into a system

$$
(\varepsilon x)^{\frac{1}{2}} \cdot x \frac{d \vec{z}}{d x}=\left(\begin{array}{cc}
0 & 1 \\
\varepsilon \tau^{-2} x-1+\frac{1}{4} \varepsilon x^{-1}-\frac{1}{4} \varepsilon x & \frac{3}{2}(\varepsilon x)^{\frac{1}{2}}
\end{array}\right) \vec{z} .
$$

To study the system (14.2) in the domains $\left(\mathrm{D}_{3}\right)$ and $\left(\mathrm{D}_{4}\right)$ :

$$
\left|\varepsilon x^{-1}\right| \leqq a_{3}, \quad 0<|\tau| \leqq b_{0}, \quad\left|\tau^{-1} x\right| \leqq c_{3}
$$

and

$$
\left|\tau x^{-1}\right| \leqq a_{4}, \quad 0<|\tau| \leqq b_{0}, \quad\left|\varepsilon \tau^{-2} x\right| \leqq c_{4},
$$

we rewrite the terms in its coefficient matrix in following two ways:

$$
\left\{\begin{aligned}
\varepsilon x & =\left\{\begin{array}{l}
\left(\varepsilon x^{-1}\right) \tau^{2}\left(\tau^{-1} x\right)^{2}, \\
\left(\tau x^{-1}\right)^{0} \tau^{2}\left(\varepsilon \tau^{-2} x\right),
\end{array}\right. \\
\varepsilon \tau^{-2} x & =\left\{\begin{array}{l}
\left(\varepsilon x^{-1}\right)\left(\tau^{-1} x\right)^{2}, \\
\left(\varepsilon \tau^{-2} x\right),
\end{array}\right. \\
\varepsilon x^{-1} & =\left\{\begin{array}{l}
\left(\varepsilon x^{-1}\right), \\
\left(\tau x^{-1}\right)^{2}\left(\varepsilon \tau^{-2} x\right) .
\end{array}\right.
\end{aligned}\right.
$$

From this relations we can easily verify that, if we substitute

$$
\varepsilon=\alpha^{2}, \quad \tau=\beta^{2}, \quad x=\xi^{2},
$$

the resulting system (14.2) satisfies the same conditions as those of Type A in both of the subdomains $\left(D_{3}\right)$ and $\left(D_{4}\right)$ except for the condition $p=0$ in the domain $\left(\mathrm{D}_{4}\right)$. However, in the latter domain, if we exchange the rôle of $\tau x^{-1}$ 
and $\varepsilon \tau^{-2} x$, for example, the condition $p>0$ will be satisfied.

Thus we have the following:

III. In the subdomain

$\left(\mathrm{D}_{3}\right)$

$$
\left|\varepsilon x^{-1}\right| \leqq a_{3}, \quad\left|\tau^{-1} x\right| \leqq c_{3}
$$

or

$\left(\mathrm{D}_{4}\right)$

$$
\left|\varepsilon \tau^{-2} x\right| \leqq a_{4}^{\prime}\left(=c_{4}\right), \quad\left|\tau x^{-1}\right| \leqq c_{4}^{\prime}\left(=a_{4}\right),
$$

the system (W) is transformed into a system of Type A if we make a change of variables :

$$
\left\{\begin{array}{l}
\vec{y}=\left(\begin{array}{cc}
1 & 0 \\
0 & \tau x^{-\frac{3}{2}}
\end{array}\right) \overrightarrow{\mathfrak{w}}, \\
\varepsilon=\alpha^{2}, \\
\tau=\beta^{2}, \\
x=\xi^{2} .
\end{array}\right.
$$

§ 15. Domain $\left(\mathbf{D}_{5}\right)$.

\section{$1^{\circ}$. Preliminary transformation.}

Noticing that the coordinate of the vertex $\mathrm{V}_{3}$ is $\left(\frac{1}{2}, 0,-1\right)$, put

$$
\vec{y}=\left(\begin{array}{cc}
1 & 0 \\
0 & \varepsilon^{\frac{1}{2}} x^{-1}
\end{array}\right) \vec{z} .
$$

Then, we can derive from (W) the following system :

$$
\tau \cdot x \frac{d \vec{z}}{d x}=\left(\begin{array}{cc}
0 & 1 \\
1-\varepsilon^{-1} \tau^{2} x^{-1}+\frac{1}{4} \tau^{2} x^{-2}-\frac{1}{4} \tau^{2} & \tau
\end{array}\right) \vec{z} .
$$

If we notice that we can write

$$
\left\{\begin{aligned}
\tau & =\left(\tau x^{-1}\right) \cdot\left(\varepsilon^{-1} \tau^{2} x^{-1}\right) \cdot\left(\varepsilon \tau^{-2} x^{2}\right), \\
\tau^{2} x^{-2} & =\left(\tau x^{-1}\right)^{2}
\end{aligned}\right.
$$

and therefore these quantities are sufficiently small in the considered subdomain

$$
\left|\tau x^{-1}\right| \leqq a_{5}, \quad\left|\varepsilon^{-1} \tau^{2} x^{-1}\right| \leqq b_{5}, \quad\left|\varepsilon \tau^{-2} x^{2}\right| \leqq c_{5},
$$

we see that the coefficient matrix of (15.2) has two distinct characteristic values which tend to \pm 1 as $\left|\tau x^{-1}\right|,\left|\varepsilon^{-1} \tau^{2} x^{-1}\right|,\left|\varepsilon \tau^{-2} x^{2}\right|$ tend to 0 .

Hence, the equation (15.2) has a form similar to Type A except for the condition $n p+n^{\prime} p^{\prime}+n^{\prime \prime} p^{\prime \prime}=0$. However, as we shall see later (in Chap. IX), it is convenient to treat this system independently from Type A.

\section{$2^{\circ}$. Reduced form of Type $B$.}

Therefore, it is sufficient to study a system of the following form:

$$
\begin{gathered}
\left(\alpha^{l} \beta^{m} \xi^{n}\right)^{p}\left(\alpha^{l^{\prime}} \beta^{m^{\prime}} \xi^{n^{\prime}}\right) p^{p^{\prime}}\left(\alpha^{l^{\prime \prime}} \beta^{m^{\prime \prime}} \xi^{n^{\prime \prime}}\right)^{p^{\prime \prime}} \cdot \xi \frac{d \overrightarrow{\mathrm{w}}}{d \xi}=A(\alpha, \beta, \xi) \overrightarrow{\mathfrak{w}} \\
\left(p>0, p^{\prime} \geqq 0, p^{\prime \prime} \geqq 0, n p+n^{\prime} p^{\prime}+n^{\prime \prime} p^{\prime \prime}=0\right),
\end{gathered}
$$


where $A(\alpha, \beta, \xi)$ is a matrix function with the same properties as in Type A.

$3^{\circ}$. We have proved the following:

IV. In the subdomain

$$
\left|\tau x^{-1}\right| \leqq a_{5}, \quad\left|\varepsilon^{-1} \tau^{2} x^{-1}\right| \leqq b_{5}, \quad\left|\varepsilon \tau^{-2} x^{2}\right| \leqq c_{5},
$$

the system (W) is transformed into a system of Type $\mathrm{B}$ if we put

$$
\left\{\begin{array}{l}
\vec{y}=\left(\begin{array}{cc}
1 & 0 \\
0 & \varepsilon^{\frac{1}{2}} x^{-1}
\end{array}\right) \overrightarrow{\mathfrak{w}}, \\
\varepsilon=\alpha, \\
\tau=\beta, \\
x=\xi .
\end{array}\right.
$$

§16. Domain $\left(\mathbf{D}_{6}\right)$.

The discussion in this domain will be quite similar as before. We consider again the system (15.2). Observing that we can write

$$
\left\{\begin{aligned}
\tau & =\varepsilon^{\frac{1}{2}} \cdot\left(\varepsilon^{-1} \tau^{2} x^{-2}\right)^{\frac{1}{2}} \cdot x, \\
\varepsilon^{-1} \tau^{2} x^{-1} & =\left(\varepsilon^{-1} \tau^{2} x^{-2}\right) \cdot x, \\
\tau x^{-1} & =\varepsilon^{\frac{1}{2}}\left(\varepsilon^{-1} \tau^{2} x^{-2}\right)^{\frac{1}{2}},
\end{aligned}\right.
$$

we see immediately that in the considered subdomain

$$
0<|\varepsilon| \leqq a_{0}, \quad\left|\varepsilon^{-1} \tau^{2} x^{-2}\right| \leqq b_{6}, \quad 0<|x| \leqq c_{0}
$$

the coefficient matrix of the system (15.2) has two distinct characteristic values and, further, is considered as a polynomial with respect to $\varepsilon^{\frac{1}{2}},\left(\varepsilon^{-1} \tau^{2} x^{-2}\right)^{\frac{1}{2}}, x$.

This proves the following:

V. In the subdomain

$$
\left|\varepsilon^{-1} \tau^{2} x^{-2}\right| \leqq b_{6}
$$

the system (W) is reduced to a system of Type B by a transformation

$$
\left\{\begin{array}{l}
\vec{y}=\left(\begin{array}{cc}
1 & 0 \\
0 & \varepsilon^{\frac{1}{2}} x^{-1}
\end{array}\right) \overrightarrow{\mathfrak{w}}, \\
\varepsilon=\alpha^{2}, \\
\tau=\beta^{2}, \\
x=\xi^{2} .
\end{array}\right.
$$

Remark. We have already seen that in the domain $\left(D_{4}\right)$ the system (14.2) is reduced to a system of Type $A$ if we exchange the rôle of $\left(\tau x^{-1}\right)$ and $\left(\varepsilon \tau^{-2} x\right)$. Consequently, as we shall see later (in Lemma 6 in Chapter IX), the asymptotic expression of a fundamental set of independent solutions defined in the domain $\left(D_{4}\right)$ is not a power series of $\left(\tau x^{-1}\right)$ but that of $\left(\varepsilon \tau^{-2} x\right)$. So, in order to obtain such an asymptotic expression which is developable in power series of $\left(\tau x^{-1}\right)$, we must study the case $p=0$ in Type A, i.e. a following system :

$$
\begin{gathered}
\left(\alpha^{l} \beta^{m^{\prime}} \xi^{n^{\prime}}\right)^{p^{\prime}}\left(\alpha^{l^{\prime \prime}} \beta^{m^{\prime \prime}} \xi^{n^{\prime \prime}}\right)^{p^{\prime \prime}} \cdot \xi \frac{d \overrightarrow{\mathfrak{w}}}{d \xi}=A(\alpha, \beta, \xi) \overrightarrow{\mathfrak{w}} \\
\left(p^{\prime} \geqq 0, \quad p^{\prime \prime} \geqq 0\right) .
\end{gathered}
$$


where at least one of $p^{\prime}$ and $p^{\prime \prime}$ is positive, and the coefficient $A(\alpha, \beta, \xi)$ is a 2 by 2 matrix function with the same asymptotic properties as the matrix $A$ appeared in Type A.

\section{Chapter IV. Study of the behavior of the solutions in the auxiliary subdomain $\left(\mathbf{D}_{7}\right)$.}

In this chapter we will show that, in order to construct an asymptotic expression of fundamental solutions in the domain $\left(D_{7}\right)$, it is sufficient to consider, in addition to a system of Type (A), following four systems called Type (C), (D), (E), (F) :

(C)

$$
\begin{aligned}
& \left(\alpha \xi^{-\mu}\right)^{p}\left(\alpha^{-\nu \xi}\right)^{q} \cdot \xi \frac{d \overrightarrow{\mathfrak{w}}}{d \xi}=C(\alpha, \beta, \xi) \overrightarrow{\mathfrak{w}} \\
& (p>0, q \geqq 0, \mu \geqq 0, \nu \geqq 0,0 \leqq \mu \nu<1,-\mu p+q \neq 0) \text {; } \\
& \left(\alpha \xi^{-\mu}\right)^{p}\left(\alpha^{-\nu \xi}\right)^{q} \cdot \xi \frac{d \overrightarrow{\mathfrak{w}}}{d \xi}=C(\alpha, \beta, \xi) \mathfrak{w} \\
& (p>0, q \geqq 0, \mu \geqq 0, \nu \geqq 0,0 \leqq \mu \nu<1,-\mu p+q=0) \text {; } \\
& \alpha^{p} \frac{d \overrightarrow{\mathfrak{w}}}{d \xi}=E(\alpha, \beta, \xi) \overrightarrow{\mathfrak{w}} \quad(p>0) ; \\
& \frac{d \overrightarrow{\mathfrak{w}}}{d \xi}=F(\alpha, \beta, \xi) \overrightarrow{\mathfrak{w}},
\end{aligned}
$$

where

i) $p$ and $q$ are integers, $\mu$ and $\nu$ are rational numbers;

ii) $C, E, F$ are matrix functions with certain properties.

\section{§17. Auxiliary subdomain $\left(D_{7}\right)$.}

The domain $\left(D_{7}\right)$ has the form

$\left(\mathrm{D}_{i}\right) \quad b \leqq\left|\varepsilon^{-1} \tau\right| \leqq B, \quad 0<|\varepsilon| \leqq a_{0}, \quad 0<|\tau| \leqq b_{0}, \quad 0<|x| \leqq c_{0}$.

If we introduce a new parameter:

$$
\tau_{1}=\varepsilon^{-1} \tau
$$

then, the domain $\left(D_{7}\right)$ is reduced to

$$
0<|\varepsilon| \leqq a_{0}, \quad b \leqq\left|\tau_{1}\right| \leqq B, \quad 0<|x| \leqq c_{0}
$$

and the equation (W) will be written as

$$
\varepsilon^{\frac{3}{2}} \tau_{1} \frac{d \vec{y}}{d x}=\left(\begin{array}{cc}
0 & 1 \\
\varepsilon x^{-2}-\varepsilon^{2} \tau_{1}^{2} x^{-3}+\frac{1}{4} \varepsilon^{3} \tau_{1}^{2} x^{-4}\left(1-x^{2}\right) & 0
\end{array}\right) \vec{y} .
$$

Therefore, it is sufficient to study the system $(\mathfrak{B})^{\prime}$ in the domain $\left(\widetilde{\mathbf{D}}_{7}\right)$. Since the equation $(\mathfrak{W})^{\prime}$ has no singularity with respect to the parameter $\tau_{1}$ in the considered domain, we shall construct a characteristic polygon with respect to $\varepsilon$ and $x$. To do this, it is sufficient to plot the following points : 


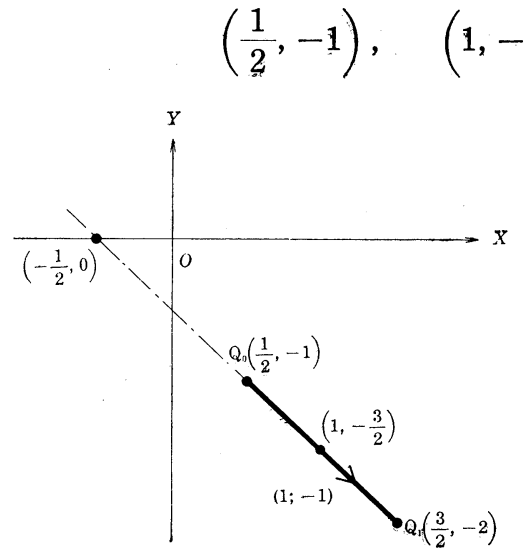

Fig. 7

in $(X, Y)$-plane, where the first three points correspond to the terms :

$$
\varepsilon x^{-2}, \quad-\tau_{1}^{2} \cdot \varepsilon^{2} x^{-3}, \frac{1}{4} \tau_{1}^{2} \cdot \varepsilon^{3} x^{-4}
$$

respectively. Then, as is shown in figure, the characteristic polygon has two vertices:

$$
\mathrm{Q}_{0}=\left(\frac{1}{2},-1\right), \quad \mathrm{Q}_{1}=\left(\frac{3}{2},-2\right) .
$$

Hence, we divide the domain $\left(\widetilde{\mathrm{D}}_{7}\right)$ into following three subdomains :

$$
\begin{gathered}
\left|\varepsilon x^{-1}\right| \leqq C_{1}^{-1} ; \\
\left|\varepsilon^{-1} x\right| \leqq c_{1} ; \\
c_{1} \leqq\left|\varepsilon^{-1} x\right| \leqq C_{1} .
\end{gathered}
$$

\section{§ 18. Domain $\left(\mathbf{H}_{1}\right)$.}

\section{$1^{\circ}$. Transformation.}

Since the coordinate of the vertex $Q_{0}$ is $\left(\frac{1}{2},-1\right)$, we make a transformation

$$
\vec{y}=\left(\begin{array}{cc}
1 & 0 \\
0 & \tau_{1} \cdot \varepsilon^{\frac{1}{2}} x^{-1}
\end{array}\right) \vec{z} \text {. }
$$

Then, the system (WB) $)^{\prime}$ will be reduced to

$$
\varepsilon \cdot x \frac{d \vec{z}}{d x}=\left(\begin{array}{cc}
0 & 1 \\
\tau_{1}^{-2}-\varepsilon x^{-1}+\frac{1}{4}\left(\varepsilon x^{-1}\right)^{2}\left(1-x^{2}\right) & \varepsilon
\end{array}\right) \vec{z} .
$$

Since we can write

$$
\varepsilon=\left(\varepsilon x^{-1}\right) \cdot x,
$$

we see at once that the matrix in (18.2) is a polynomial of $\varepsilon x^{-1}$ and $x$ whose coefficients are also polynomials of $\tau_{1}^{-1}$. Since $c_{0}$ and $1 / C_{1}$ are supposed to be sufficiently small, the coefficient matrix of (18.2) has two distinct characteristic values and they tend to $\pm \tau_{1}^{-1}$ as $\left|\varepsilon x^{-1}\right|$ and $|x|$ tend to 0 .

\section{$2^{\circ}$. Reduced form of Type $D$.}

Therefore, to study (18.2), it is sufficient to consider a more general system of the following form :

$$
\left(\alpha \xi^{-\mu}\right)^{p}\left(\alpha^{-\nu \xi}\right)^{q} \cdot \xi \frac{d \overrightarrow{\mathfrak{w}}}{d \xi}=C(\alpha, \beta, \xi) \overrightarrow{\mathfrak{w}}
$$

where we suppose that

1) $p$ is a positive integer and $q$ is a nonnegative integer; 
2) $\mu$ and $\nu$ are nonnegative rational numbers such that $0 \leqq \mu \nu<1$;

3) $-\mu p+q=0$;

4) $C(\alpha, \beta, \xi)$ is a matrix function holomorphic and bounded in $(\alpha, \beta, \xi)$ for

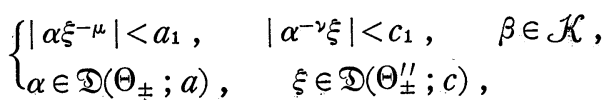

and, moreover, admitting a uniform asymptotic expansion in powers of $\left(\alpha \xi^{-\mu}\right)$ :

$$
C(\alpha, \beta, \xi) \sim \sum_{h=0}^{\infty}\left(\alpha \xi^{-\mu}\right)^{h} C_{h}\left(\beta, \alpha^{-\nu \xi}\right)
$$

as $\alpha$ tends to 0 in the domain (18.3);

5) $C_{h}(\beta, \zeta)$ are matrix functions which are holomorphic and bounded in $(\beta, \zeta)$ for

$$
\beta \in \mathcal{K}, \quad \zeta \in \mathscr{D}\left(\Delta_{ \pm} ; d\right)
$$

and admit uniform asymptotic expansions in powers of $\zeta$ :

$$
C_{h}(\beta, \zeta) \sim \sum_{k=0}^{\infty} \zeta^{k} C_{h k}(\beta)
$$

as $\zeta$ tends to 0 in the domain (18.4), where the coefficients $C_{k i k}(\beta)$ are functions holomorphic and bounded in $\beta$ for

$$
\beta \in \mathcal{K}
$$

6) the matrix $C_{00}(\beta)$ has two distinct characteristic values in the domain (18.5);

7) $\mathcal{K}$ is a simply connected, open and bounded region which does not contain the origin. Moreover, for the values of $(\alpha, \xi)$ in (18.3), the value of $\zeta=\alpha^{-\nu \xi}$ is always contained in the domain $\zeta \in \mathfrak{D}\left(\Delta_{ \pm} ; d\right)$.

The following fact should be noticed:

If we put $\mu=1, \nu=0, p=q=1$ and $\mathcal{K}=\left\{\beta:|\arg \beta|<\Theta^{\prime}, b<|\beta|<B\right\}\left(\Theta^{\prime}>\pi\right)$, the system of Type D will be reduced to a system of the form (18.2).

$3^{\circ}$. Thus we have proved the following:

VI. Consider the subdomain

$$
C_{1} \leqq\left|\varepsilon^{-1} x\right|, \quad b \leqq\left|\varepsilon^{-1} \tau\right| \leqq B .
$$

Then, the system (W)' will be reduced to a system of Type $\mathrm{D}$ if we put

$$
\left\{\begin{array}{l}
\vec{y}=\left(\begin{array}{cc}
1 & 0 \\
0 & \tau_{1} \varepsilon^{\frac{1}{2}} x^{-1}
\end{array}\right) \overrightarrow{\mathfrak{w}}, \\
\varepsilon=\alpha, \\
\tau_{1}\left(=\varepsilon^{-1} \tau\right)=\beta, \\
x=\xi .
\end{array}\right.
$$

§19. Domain $\left(\mathbf{H}_{2}\right)$.

$\mathbf{1}^{\circ}$. Transformation.

We make a transformation

$$
\vec{y}=\left(\begin{array}{cc}
1 & 0 \\
0 & \tau_{1} \cdot \varepsilon^{\frac{3}{2}} x^{-2}
\end{array}\right) \vec{z}
$$


noticing that the coordinate of the vertex $Q_{1}$ is $\left(\frac{3}{2},-2\right)$. Then, we have the following system :

$$
x \cdot x \frac{d \vec{z}}{d x}=\left(\begin{array}{cc}
1 & 0 \\
\tau_{1}^{-2}\left(\varepsilon^{-1} x\right)^{2}-\left(\varepsilon^{-1} x\right)+\frac{1}{4}\left(1-x^{2}\right) & 2 x
\end{array}\right) \vec{z} .
$$

Observing that we can write

$$
x=\varepsilon\left(\varepsilon^{-1} x\right),
$$

it is easily seen that the coefficient matrix of the system (19.2) is regarded as a polynomial of $\varepsilon$ and $\varepsilon^{-1} x$ whose coefficients are polynomials of $\tau_{1}^{-1}$.

Since the quantities $a_{0}$ and $c_{1}$ (appearing in the domain $\left(\mathrm{H}_{2}\right)$ ) are supposed to be sufficiently small, the coefficient matrix has two distinct characteristic values in the domain $\left(\mathrm{H}_{2}\right)$. Hence, by the same reasoning as in the subdomain $\left(\mathrm{H}_{1}\right)$, we see that the system (19.2) has a form similar to Type $\mathrm{D}$ (where we have to put $\mu=0$ ) except for the condition $-\mu p+q \neq 0$. However, as we shall see later (in Chap. IX), it is convenient to treat this system independently from Type D.

\section{$2^{\circ}$. Reduced form of Type C.}

Therefore, it is sufficient to study a system of the following form :

$$
\begin{aligned}
& \left(\alpha \xi^{-\mu}\right)^{p}\left(\alpha^{-\nu \xi}\right)^{q} \cdot \xi \frac{d \overrightarrow{\mathfrak{w}}}{d \xi}=C(\alpha, \beta, \xi) \overrightarrow{\mathfrak{w}} \\
& \quad(p>0, q \geqq 0, \mu \geqq 0, \nu \geqq 0,0 \leqq \mu \nu<1,-\mu p+q \neq 0) .
\end{aligned}
$$

The coefficient $C(\alpha, \beta, \xi)$ is a matrix with the same properties as the matrix $C$ appeared in Type D.

$3^{\circ}$. We have proved the following:

VII. In the subdomain

$$
\left|\varepsilon^{-1} x\right| \leqq c_{1}, \quad b \leqq\left|\varepsilon^{-1} \tau\right| \leqq B,
$$

the $(\mathfrak{B})^{\prime}$ will be reduced to a system of Type $\mathrm{C}$ by making a change of variables:

$$
\left\{\begin{array}{l}
\vec{y}=\left(\begin{array}{cc}
1 & 1 \\
0 & \tau_{1} \cdot \varepsilon^{\frac{3}{2}} x^{-1}
\end{array}\right) \overrightarrow{\mathfrak{w}}, \\
\varepsilon=\alpha, \\
\tau_{1}\left(=\varepsilon^{-1} \tau\right)=\beta, \\
x=\xi .
\end{array}\right.
$$

§ 20. Domain $\left(\mathbf{H}_{3}\right)$.

In order to investigate the behavior of solutions of the system (W)' in the subdomain $\left(\mathrm{H}_{3}\right)$, we make a transformation

$$
\left\{\begin{aligned}
\vec{y} & =\left(\begin{array}{cc}
1 & 0 \\
0 & \tau_{1} \cdot \varepsilon^{-\frac{1}{2}}
\end{array}\right) \vec{z}, \\
\varepsilon^{-1} x & =x_{1}
\end{aligned}\right.
$$


noticing that the coordinate of the point (at which the straight line passing through the points $\mathrm{Q}_{0}$ and $\mathrm{Q}_{1}$ intersects with $X$-axis) is $\left(-\frac{1}{2}, 0\right)$ and the inverse of the directional parameter of the side $\mathrm{Q}_{0} \mathrm{Q}_{1}$ is equal to -1 (See Fig. 7).

Then, we can derive from the system (W)' the following:

$$
\varepsilon \frac{d \vec{z}}{d x_{1}}=\left(\begin{array}{cc}
0 & 1 \\
\tau_{1}^{-2} x_{1}^{-2}-x_{1}^{-3}+\frac{1}{4} x_{1}^{-4}-\frac{1}{4} \varepsilon^{2} x_{1}^{-2} & 0
\end{array}\right) \vec{z}
$$

and the domain $\left(\mathrm{H}_{3}\right)$, is reduced to

$$
0<|\varepsilon| \leqq a_{0}, \quad b \leqq\left|\tau_{1}\right| \leqq B, \quad c_{1} \leqq\left|x_{1}\right| \leqq C_{1} .
$$

The coefficient matrix of (20.2) is a polynomial of $\varepsilon$ whose coefficients also are polynomials of $\tau_{1}^{-1}$ and $x_{1}^{-1}$. Since $a_{0}$ is supposed to be sufficiently small, the principal matrix will be written as

$$
A\left(0, \tau_{1}, x_{1}\right)=\left(\begin{array}{cc}
0 & 1 \\
\tau_{1}^{-2} x_{1}^{-4}\left(x_{1}^{2}-\tau_{1}^{2} x_{1}+\frac{1}{4} \tau_{1}^{2}\right) & 0
\end{array}\right) .
$$

Then, we see immediately that this matrix has two distinct characteristic values in a domain

$$
b \leqq\left|\tau_{1}\right| \leqq B, \quad c_{1} \leqq\left|x_{1}\right| \leqq C_{1}, \quad\left|x_{1}-f_{ \pm}\left(\tau_{1}\right)\right| \geqq c_{2},
$$

where $f_{ \pm}\left(\tau_{1}\right)$ are the two roots of an algebraic equation of $x_{1}$ :

$$
x_{1}^{2}-\tau_{1}^{2} x_{1}+\frac{1}{4} \tau_{1}^{2}=0 .
$$

By a simple calculation we have

$$
f_{ \pm}\left(\tau_{1}\right)=\frac{1}{2} \tau_{1}\left(\tau_{1} \pm \sqrt{\tau_{1}^{2}-1}\right) .
$$

It is easily verified that the functions $f_{ \pm}\left(\tau_{1}\right)$ never vanish and are bounded for

$$
b \leqq\left|\tau_{1}\right| \leqq B \text {. }
$$

Therefore, since the quantities $1 / C_{1}$ and $c_{1}$ are supposed to be sufficiently small, we can suppose that

$$
c_{1}<\left|f_{ \pm}\left(\tau_{1}\right)\right|<C_{1}
$$

if $\tau_{1}$ belongs to the domain (20.4).

We divide the domain $\left(\widetilde{\mathrm{H}}_{3}\right)$ into following two subdomains :

$$
\begin{aligned}
& \left|x_{1}-f_{ \pm}\left(\tau_{1}\right)\right| \geqq c_{2}, \quad c_{1} \leqq\left|x_{1}\right| \leqq C_{1} ; \\
& \left|x_{1}-f_{ \pm}\left(\tau_{1}\right)\right| \leqq c_{2} .
\end{aligned}
$$

$2^{\circ}$. Reduced form of Type $E$.

In order to investigate the behavior of solutions in the domain $\left(\mathrm{H}_{4}\right)$, it is sufficient to study a little more general system of the following form:

$$
\alpha^{p} \frac{d \overrightarrow{\mathfrak{w}}}{d \xi}=E(\alpha, \beta, \xi) \overrightarrow{\mathfrak{w}},
$$


where we suppose that

1) $p$ is a positive integer;

2) $E(\alpha, \beta, \xi)$ is a matrix function holomorphic and bounded in $(\alpha, \beta, \xi)$ for

$$
\alpha \in \mathfrak{D}\left(\Theta_{ \pm} ; a\right), \quad \beta \in \mathcal{K}_{1}, \quad \xi \in \mathcal{K}_{2}
$$

and, moreover, admitting a uniform asymptotic expansion of a form

$$
E(\alpha, \beta, \xi) \sim \sum_{h=0}^{\infty} \alpha^{h} E_{h}(\beta, \xi)
$$

as $\alpha$ tends to 0 in the domain (20.5):

3) $E_{h}(\beta, \xi)$ are matrix functions holomorphic and bounded in $(\beta, \xi)$ for

$$
\beta \in \mathcal{K}_{1}, \quad \xi \in \mathcal{K}_{2} ;
$$

4) $E_{0}(\beta, \xi)$ has two distinct characteristic values in (20.6);

5) $\mathcal{K}_{1}$ and $\mathcal{K}_{2}$ are both simply connected, bounded and open domains.

Remarks. ( $i$ ) In the present case, it is sufficient to put:

$$
\begin{aligned}
& \mathcal{K}_{1}=\left\{\beta: b<|\beta|<B, \Theta_{-}^{\prime}<\arg \beta<\Theta_{+}^{\prime}\right\}, \\
& \mathcal{K}_{2}=\left\{\xi ; c^{\prime}<|\xi|<C^{\prime}, \Theta_{-}^{\prime \prime}<\arg \xi<\Theta_{+}^{\prime \prime}\right\},
\end{aligned}
$$

where we take $\left(c^{\prime}=c_{1}, C^{\prime}=C_{1}\right)$ or $\left(c^{\prime}=c_{1}, C^{\prime}=\left|f_{ \pm}\left(\tau_{1}\right)\right|-c_{2}\right.$ or $\left.c^{\prime}=\left|f_{ \pm}\left(\tau_{1}\right)\right|+c_{2}, C^{\prime}=C_{1}\right)$

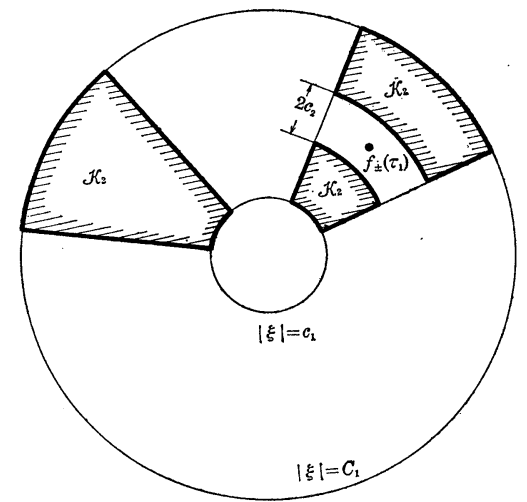

Fig. 8 according as $\arg \xi \neq \arg f_{ \pm}\left(\tau_{1}\right)$ or not (See Fig. 8).

(ii) However, in certain cases (for example, See $2^{\circ}$ in $\S 25$ or $\left.\S 38\right), \mathcal{K}_{1}$ and $\mathcal{K}_{2}$ should be taken as follows:

$$
\begin{aligned}
& \mathcal{K}_{1}=(\beta:|\beta|<b\}, \\
& \mathcal{K}_{2}=\{\xi:|\xi|<c\} .
\end{aligned}
$$

In this case, the matrices $E_{h}(\beta, \xi)$ admit uniform asymptotic expansions in powers of $\beta$.

$3^{\circ}$. Thus the following proposition has been established:

VIII. If we put

$$
\left\{\begin{array}{l}
\vec{y}=\left(\begin{array}{cc}
1 & 0 \\
0 & \tau_{1} \cdot \varepsilon^{-\frac{1}{2}}
\end{array}\right) \mathfrak{w}, \\
\varepsilon=\alpha, \\
\tau_{1}\left(=\varepsilon^{-1} \tau\right)=\beta, \\
x_{1}\left(=\varepsilon^{-1} x\right)=\xi,
\end{array}\right.
$$

the system $(\mathfrak{B})^{\prime}$ will be reduced to a system of Type $\mathrm{E}$ in the subdomain

$$
\left\{\begin{array}{l}
b \leqq\left|\varepsilon^{-1} \tau\right| \leqq B, \quad c_{1} \leqq\left|\varepsilon^{-1} x\right| \leqq C_{1}, \\
\left|\varepsilon^{-1} x-f_{ \pm}\left(\varepsilon^{-1} \tau\right)\right| \geqq c_{2}
\end{array}\right.
$$

§ 21. Domain $\left(\mathbf{H}_{5}\right)$.

We consider again the system (20.2). In order to investigate the behavior 
of solutions of (20.2) in the subdomain $\left(\mathrm{H}_{5}\right)$, we introduce a new independent variable :

$$
x_{2}=x_{1}-f_{ \pm}\left(\tau_{1}\right) .
$$

Then, the system (20.2) becomes

$$
\varepsilon \frac{d \vec{z}}{d x_{2}}=\left(\begin{array}{cc}
0 & 1 \\
\frac{\left(2 f_{ \pm}\left(\tau_{1}\right)-\tau_{1}^{2}\right) x_{2}+x_{2}^{2}}{\tau_{1}^{2}\left(f_{ \pm}\left(\tau_{1}\right)+x_{2}\right)^{4}}-\frac{\varepsilon^{2}}{4\left(f_{ \pm}\left(\tau_{1}\right)+x_{2}\right)^{2}} & 0
\end{array}\right) \vec{z}
$$

and the considered domain is written as

$$
0<|\varepsilon| \leqq a_{0}, \quad b \leqq\left|\tau_{1}\right| \leqq B, \quad\left|x_{2}\right| \leqq c_{2} .
$$

As we have already seen, the functions $f_{ \pm}\left(\tau_{1}\right)$ never vanish. For the system (21.2), $\varepsilon=0$ is a singularity and $x_{2}=0$ may possibly be a turning point. Hence, we construct a characteristic polygon with respect to $\varepsilon$ and $x_{2}$.

The characteristic polygon has two vertices

$$
\mathrm{Q}_{0}=\left(0, \frac{1}{2}\right), \quad \mathrm{Q}_{1}(=\mathrm{R})=(1,-1)
$$

as is shown in figure.

We divide the domain $\left(\widetilde{\mathrm{H}}_{5}\right)$ into following two subdomains :

$$
\begin{aligned}
& \left|\varepsilon^{-\frac{2}{3}} x_{2}\right| \leqq D_{6} \\
& \left|\varepsilon x_{2}^{-\frac{3}{2}}\right| \leqq d_{7}
\end{aligned}
$$

\section{§ 22. Domain $\left(\mathbf{H}_{6}\right)$.}

\section{$1^{\circ}$. Transformation.}

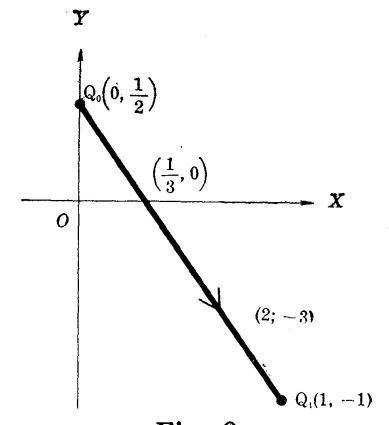

Fig. 9

Noticing that the coordinate of the point (at which the straight line passing through the points $\mathrm{Q}_{0}$ and $\mathrm{Q}_{1}$ intersects with $X$-axis) is $\left(\frac{1}{3}, 0\right)$ and the inverse of the directional parameter of the side $Q_{0} Q_{1}$ is equal to $-\frac{2}{3}$ (See Fig. 9), let us make a transformation:

$$
\left\{\begin{aligned}
\vec{z} & =\left(\begin{array}{ll}
1 & 0 \\
0 & \varepsilon^{\frac{1}{3}}
\end{array}\right) \vec{u} \\
\varepsilon^{-\frac{2}{3}} x_{2} & =x_{3}
\end{aligned}\right.
$$

Then, we have, from (21.2),

$$
\frac{d \vec{u}}{d x_{3}}=\left(\begin{array}{cc}
0 & 1 \\
L\left(\varepsilon, \tau_{1}, x_{3}\right) & 0
\end{array}\right) \vec{u}
$$

where

$$
L=\frac{\left(2 f_{ \pm}\left(\tau_{1}\right)-\tau_{1}^{2}\right) x_{3}+\varepsilon^{\frac{2}{3}} x_{3}^{2}}{\tau_{1}^{2}\left(f_{ \pm}\left(\tau_{1}\right)+\varepsilon^{\frac{2}{3}} x_{3}\right)^{4}}-\frac{\varepsilon^{\frac{4}{3}}}{4\left(f_{ \pm}\left(\tau_{1}\right)+\varepsilon^{\frac{2}{3}} x_{3}\right)^{2}} .
$$

Therefore, by substituting $\varepsilon^{\frac{1}{3}}$ by $\varepsilon_{1}$, we see that the coefficient matrix of the system (22.2) is holomorphic and bounded in $\left(\varepsilon_{1}, \tau_{1}, x_{3}\right)$ for 
$\left(\tilde{\mathrm{H}}_{6}\right)$

$$
\left|\varepsilon_{1}\right| \leqq a_{0}^{\frac{1}{3}}, \quad b \leqq\left|\tau_{1}\right| \leqq B, \quad\left|x_{3}\right| \leqq D_{6}
$$

(except for two branch points $\tau_{1}= \pm 1$ ), provided that, no matter how $D_{6}$ is large, $a_{0}$ is sufficiently small. Moreover, the coefficient matrix is developable in a uniformly convergent power series of $\varepsilon_{1}$ :

$$
\sum_{h=0}^{\infty} \varepsilon_{1}^{h} A_{h}\left(\tau_{1}, x_{3}\right),
$$

where $A_{h}\left(\tau_{1}, x_{3}\right)$ are polynomials of $x_{3}$ whose coefficients are functions holomorphic and bounded in $\tau_{1}$ for

$$
b \leqq\left|\tau_{1}\right| \leqq B
$$

(except for two branch points $\tau_{1}= \pm 1$ ).

\section{$2^{\circ}$. Reduced form of Type $F$.}

Therefore, in stead of (22.2), it is sufficient to study a system of the form:

$$
\frac{d \overrightarrow{\mathfrak{w}}}{d \xi}=F(\alpha, \beta, \xi) \overrightarrow{\mathfrak{w}},
$$

where we suppose that

1) $F(\alpha, \beta, \xi)$ is a matrix function holomorphic and bounded in $(\alpha, \beta, \xi)$ for

$$
\alpha \in \mathfrak{D}\left(\Theta_{ \pm} ; a\right), \quad \beta \in \mathcal{K}, \quad \xi \in \mathscr{D}\left(\Theta_{ \pm}^{\prime \prime} ; c\right)
$$

and, moreover, admitting a uniform asymptotic expansion in powers of $\alpha$ :

$$
F(\alpha, \beta, \xi) \sim \sum_{h=0}^{\infty} \alpha^{h} F_{h}(\beta, \xi)
$$

as $\alpha$ tends to 0 in the domain (22.4);

2) $F_{h}(\beta, \xi)$ are matrix functions holomorphic and bounded in $(\beta, \xi)$ for

$$
\beta \in \mathcal{K}, \quad \xi \in \mathbb{D}\left(\Theta_{ \pm}^{\prime \prime} ; c\right)
$$

and asymptotically developable in powers of $\xi$ :

$$
F_{h}(\beta, \xi) \sim \sum_{k=0}^{\infty} \xi^{k} F_{h k}(\beta),
$$

uniformly valid for $\beta \in \mathcal{K}$, as $\xi$ tends to 0 in (22.5);

3) $F_{h k}(\beta)$ are matrix functions holomorphic and bounded in $\beta$ for

$$
\beta \in \mathcal{K} ;
$$

4) $\mathcal{K}$ denotes an open, bounded and simply connected domain.

$\mathbf{3}^{\circ}$. Thus we can state the following:

IX. By making a change of variables

$$
\left\{\begin{array}{l}
\vec{y}=\left(\begin{array}{cc}
1 & 0 \\
0 & \tau_{1} \varepsilon^{-\frac{1}{2}} \cdot \varepsilon^{\frac{1}{3}}
\end{array}\right) \overrightarrow{\mathfrak{w}}, \\
\varepsilon_{1}\left(=\varepsilon^{\frac{1}{3}}\right)=\alpha, \\
\tau_{1}\left(=\varepsilon^{-1} \tau\right)=\beta, \\
x_{3}\left(=\varepsilon^{-\frac{2}{3}} x_{2}=\varepsilon^{-\frac{2}{3}}\left[\varepsilon^{-1} x-f_{ \pm}\left(\varepsilon^{-1} \tau\right)\right]\right)=\xi,
\end{array}\right.
$$


the system $(\mathfrak{W})^{\prime}$ will be reduced to a system of Type $\mathrm{F}$ in the domain $\left(\widetilde{\mathrm{H}}_{6}\right)$ or

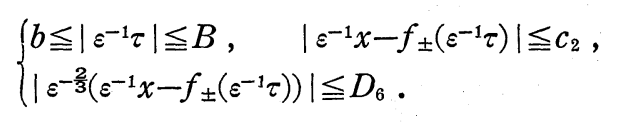

\section{§ 23. Domain $\left(\mathbf{H}_{7}\right)$.}

We should first notice that the domain $\left(\mathrm{H}_{7}\right)$ corresponds to the vertex $\mathrm{Q}_{0}$ of the characteristic polygon of the system (21.2). So let us make a transformation

$$
\vec{z}=\left(\begin{array}{cc}
1 & 0 \\
0 & x_{2}^{\frac{1}{2}}
\end{array}\right) \vec{u},
$$

then, the system (21.2) is changed into a system

$$
\left(\varepsilon x_{2}^{-\frac{3}{2}}\right) \cdot x_{2} \frac{d \vec{u}}{d x_{2}}=\left(\begin{array}{cc}
0 & 1 \\
L\left(\varepsilon, \tau_{1}, x_{2}\right) & -\frac{1}{2}\left(\varepsilon x_{2}^{-\frac{3}{2}}\right)
\end{array}\right) \vec{u},
$$

where

$$
L=\frac{2 f_{ \pm}\left(\tau_{1}\right)-\tau_{1}^{2}+x_{2}}{\tau_{1}^{2}\left(f_{ \pm}\left(\tau_{1}\right)+x_{2}\right)^{4}}-\frac{\varepsilon^{2} x_{2}^{-1}}{4\left(f_{ \pm}\left(\tau_{1}\right)+x_{2}\right)^{2}} .
$$

Therefore, the coefficient matrix of (23.2) is a polynomial of $\left(\varepsilon x_{2}^{-\frac{3}{2}}\right)$ and its coefficients are functions developable in uniformly convergent power series of $x_{2}$ whose coefficients are functions holomorphic and bounded in $\tau_{1}$ for

$$
b \leqq\left|\tau_{1}\right| \leqq B
$$

(except for two branch points $\tau_{1}= \pm 1$ ). Since, by (20.3),

$$
2 f_{ \pm}\left(\tau_{1}\right)-\tau_{1}^{2}= \pm\left(\tau_{1}^{2}-1\right)^{\frac{1}{2}}
$$

we see that the coefficient matrix tends to

$$
A\left(0, \tau_{1}, 0\right)=\left(\begin{array}{cc}
0 & 1 \\
\pm \frac{\sqrt{\tau_{1}^{2}-1}}{\tau_{1}^{2} f_{ \pm}\left(\tau_{1}\right)^{4}} & 0
\end{array}\right)
$$

as $\left(\varepsilon x_{2}^{-\frac{3}{2}}\right)$ and $x_{2}$ tend to 0 .

Therefore, if $\tau_{1} \neq \pm 1$, the matrix $A\left(0, \tau_{1}, 0\right)$ and, consequently, the coefficient matrix itself has two distinct characteristic values for

$$
\left|\varepsilon x_{2}^{-\frac{3}{2}}\right| \leqq d_{7}, \quad b \leqq\left|\tau_{1}\right| \leqq B, \quad\left|\tau_{1} \pm 1\right| \geqq b_{1} .
$$

Keeping this fact in mind, we divide the domain $\left(\mathrm{H}_{7}\right)$ into following two subdomains :

$$
\begin{array}{ll}
\left|\tau_{1} \pm 1\right| \geqq b_{1}, & \left|\varepsilon x_{2}^{-\frac{3}{2}}\right| \leqq d_{7}
\end{array} \mid
$$

Then we at once see that, in the subdomain $\left(\mathrm{H}_{8}\right)$, the system (23.2) is a system of Type $\mathrm{C}$ (where $\nu=0, q=0$ and $\mathcal{K}$ has a form similar to the domain $\mathcal{K}_{2}$ stated in Remark (i) in $\S 20$ ). 
Thus we have the following:

X. In the subdomain

$\left(\mathrm{H}_{8}\right)$

$$
\left\{\begin{array}{l}
b \leqq\left|\varepsilon^{-1} \tau\right| \leqq B, \quad\left|\varepsilon^{-1} x-f_{ \pm}\left(\varepsilon^{-1} \tau\right)\right| \leqq c_{2} \\
\left|\varepsilon\left(\varepsilon^{-1} x-f_{ \pm}\left(\varepsilon^{-1} \tau\right)\right)^{-\frac{3}{2}}\right| \leqq d_{7} \\
\left|\varepsilon^{-1} \tau \pm 1\right| \geqq b_{1}
\end{array}\right.
$$

a transformation

$$
\left\{\begin{array}{l}
\vec{y}=\left(\begin{array}{cc}
1 & 0 \\
0 & \tau_{1} \varepsilon^{-\frac{1}{2}} \cdot x_{2}^{\frac{1}{2}}
\end{array}\right) \overrightarrow{\mathfrak{w}}, \\
\varepsilon=\alpha, \\
\tau_{1}\left(=\varepsilon^{-1} \tau\right)=\beta, \\
x_{2}\left(=\varepsilon^{-1} x-f_{ \pm}\left(\varepsilon^{-1} \tau\right)\right)=\xi
\end{array}\right.
$$

reduces the system (MB)' to a system of Type C.

\section{§ 24. Domain $\left(\mathbf{H}_{9}\right)$.}

\section{$1^{\circ}$. Reduction of the domain $\left(\mathbf{H}_{9}\right)$.}

It remains only to study the behavior of solutions of the system (23.2) in the subdomain $\left(\mathrm{H}_{9}\right)$ :

$$
\left\{\begin{array}{l}
0<|\varepsilon| \leqq a_{0}, \quad\left|\tau_{1} \pm 1\right| \leqq b_{1}, \quad\left|x_{2}\right| \leqq c_{2}, \\
\left|\varepsilon x_{2}^{-\frac{3}{2}}\right| \leqq d_{7}
\end{array}\right.
$$

Clearly, this domain is a subdomain of the domain

$$
\left\{\begin{array}{l}
0<|\varepsilon| \leqq a_{0}, \quad\left|\tau_{1} \pm 1\right| \leqq b_{1}, \quad\left|x_{1}-f_{ \pm}\left(\tau_{1}\right)\right| \leqq c_{2}, \\
b \leqq\left|\tau_{1}\right| \leqq B, \quad c_{1} \leqq\left|x_{1}\right| \leqq C_{1} .
\end{array}\right.
$$

Therefore, it is sufficient to investigate the behavior of solutions of the system (20.2) in the domain $\left(\mathrm{H}_{9}^{\prime}\right)$.

However, as $\tau_{1}$ is sufficiently near to 1 or -1 , the values of the functions $f_{ \pm}\left(\tau_{1}\right)$ are contained in a small neighborhood of the point $1 / 2$ (See (20.3)). From this we see immediately that the domain $\left(\mathrm{H}_{9}^{\prime}\right)$ is equivalent to the domain

$$
0<|\varepsilon| \leqq a_{0}, \quad\left|\tau_{1} \pm 1\right| \leqq b_{1}, \quad\left|x_{1}-\frac{1}{2}\right| \leqq c_{2} .
$$

Therefore, it suffices to study the system (20.2) in the domain $\left(\mathrm{H}_{9}^{\prime \prime}\right)$. To do this, we introduce a new parameter $\tau_{2}$ and a new independent variable $x_{2}$ in a following way :

$$
\left\{\begin{array}{l}
\tau_{2}=\tau_{1} \pm 1\left(=\varepsilon^{-1} \tau \pm 1\right), \\
x_{2}=x_{1}-\frac{1}{2}\left(=\varepsilon^{-1} x-\frac{1}{2}\right) .
\end{array}\right.
$$

Then, the domain $\left(\mathrm{H}_{9}^{\prime \prime}\right)$ is written as

$$
0<|\varepsilon| \leqq a_{0}, \quad\left|\tau_{2}\right| \leqq b_{1}, \quad\left|x_{2}\right| \leqq c_{2} .
$$

After a simple calculation, we see that the system (20.2) is reduced to 


$$
\varepsilon \frac{d \vec{z}}{d x_{2}}=\left(\begin{array}{cc}
0 & 1 \\
L\left(\varepsilon, \tau_{2}, x_{2}\right) & 0
\end{array}\right) \vec{z},
$$

where

$$
L=\frac{16 x_{2}^{2}-16\left(\tau_{2}^{2} \pm 2 \tau_{2}\right) x_{2}-4\left(\tau_{2}^{2} \pm 2 \tau_{2}\right)}{\left(1 \pm \tau_{2}\right)^{2}\left(1+2 x_{2}\right)^{4}}-\frac{\varepsilon^{2}}{\left(1+2 x_{2}\right)^{2}} .
$$

$2^{\circ}$. Characteristic polyhedron of the system (24.2).

The coefficient matrix of (24.2) is a polynomial of $\varepsilon$ whose coefficient are functions developable in uniformly convergent double power series of $\tau_{2}$ and $x_{2}$. $x_{2}=0$ may possibly be a turning point of the system (24.2).

Let us construct a characteristic polyhedron of $(24.2)$ as in $\S 8$. To do this, we plot the following points :

$$
(0,0,1), \quad\left(0,-\frac{1}{2}, 0\right), \quad(1,0,-1)
$$

in $(X, Y, Z)$-space, where the first two points correspond to the terms $16 x_{2}^{2}$ and $\mp 8 \tau_{2}$ respectively. However, since the coefficient matrix is holomorphic at $x_{2}=0$, it is sufficient to consider a modified characteristic polyhedron. Then, as is shown in figure, the modified characteristic polyhedron consists of three vertices :

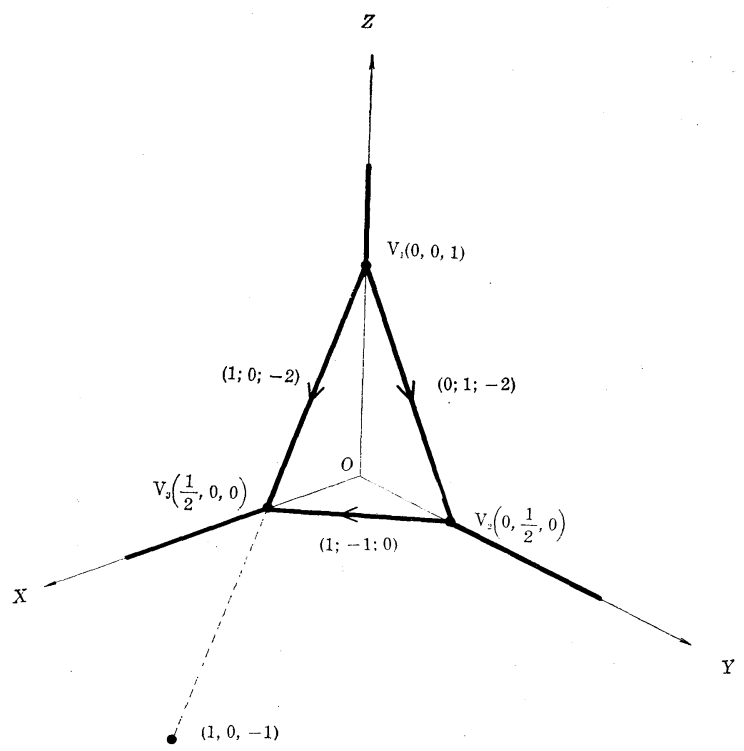

Fig. 10

$$
\mathrm{V}_{1}=(0,0,1), \quad \mathrm{V}_{2}=\left(0, \frac{1}{2}, 0\right), \quad \mathrm{V}_{3}=\left(\frac{1}{2}, 0,0\right)
$$

and of four sides. Further, at each vertex just three sides meet.

$3^{\circ}$. Division of the domain $\left(\widetilde{\mathbf{H}}_{9}^{\prime \prime}\right)$.

As is easily seen from the figure, there exists only one characteristic trigonal pyramid at each vertex. By an elementary calculation we see that 
the directional parameters of the three edges of the three characteristic trigonal pyramids are given by

$$
\begin{array}{lrr}
(1 ; 0 ;-2), & (0 ; 1 ;-2), & (0 ; 0 ; 1) ; \\
(1 ;-1 ; 0), & (0 ; 1 ; 0), & (0 ;-1 ; 2) ; \\
(1 ; 0 ; 0), & (-1 ; 1 ; 0), & (-1 ; 0 ; 2),
\end{array}
$$

which correspond to the vertices $\mathrm{V}_{1}, \mathrm{~V}_{2}$ and $\mathrm{V}_{3}$ respectively. Thus the principal subdomains are written as

$$
\begin{aligned}
& \left(\mathfrak{S}_{10}\right) \quad\left|\varepsilon x_{2}^{-2}\right| \leqq a_{10}, \quad\left|\tau_{2} x_{2}^{-2}\right| \leqq b_{10} ; \\
& \left(\mathfrak{S}_{11}\right) \quad\left|\varepsilon \tau_{2}^{-1}\right| \leqq a_{11}, \quad\left|\tau_{2}^{-1} x_{2}^{2}\right| \leqq c_{11} \text {; } \\
& \left(\mathfrak{H}_{12}\right) \quad\left|\varepsilon^{-1} \tau_{2}\right| \leqq b_{12}, \quad\left|\varepsilon^{-1} x_{2}^{2}\right| \leqq c_{12} \text {, }
\end{aligned}
$$

which correspond to characteristic trigonal pyramids at the vertices $V_{1}, V_{2}$ and $\mathrm{V}_{3}$ respectively. However, as we shall see later, the quantities $b_{12}$ and $c_{12}$ can be chosen as large as we want. So, in place of the domain $\left(\mathfrak{S}_{12}\right)$, we consider a following domain:

$$
\left|\varepsilon^{-1} \tau_{2}\right| \leqq B_{12}, \quad\left|\varepsilon^{-1} x_{2}^{2}\right| \leqq C_{12} .
$$

We add a following auxiliary subdomain:

$$
c_{13} \leqq\left|\tau_{2}^{-\frac{1}{2}} x_{2}\right| \leqq C_{13}
$$

\section{$4^{\circ}$. Possibility of covering.}

We can assert that the union of the subdomains $\left(\mathfrak{S}_{10}\right),\left(\mathfrak{S}_{11}\right),\left(\mathfrak{S}_{12}^{\prime}\right)$ and $\left(\mathfrak{S}_{13}\right)$ covers the domain $\left(\widetilde{\mathrm{H}}_{9}^{\prime \prime}\right)$ without lacuna.

This proposition follows at once from the following :

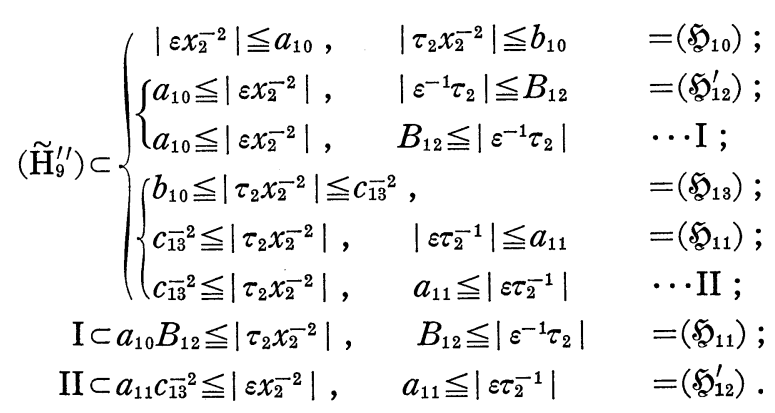

Here we suppose that the quantity $a_{10} B_{12}$ is sufficiently large.

§25. Principal subdomains $\left(\mathfrak{S}_{10}\right),\left(\mathfrak{S}_{11}\right),\left(\mathfrak{S}_{12}^{\prime}\right)$.

To investigate the behavior of the solutions of the system (24.2) in the principal subdomains, we can apply the same reasonings as in Chapter III.

$1^{\circ}$. Domain $\left(\mathfrak{S}_{10}\right)$.

Put

$$
\vec{z}=\left(\begin{array}{ll}
1 & 0 \\
0 & x_{2}
\end{array}\right) \vec{u} .
$$

Then, the system (24.2) will be reduced to 


$$
\left(\varepsilon x_{2}^{-2}\right) \cdot x_{2} \frac{d \vec{u}}{d x_{2}}=\left(\begin{array}{cc}
0 & 1 \\
x_{2}^{-2} L & -\left(\varepsilon x_{2}^{-2}\right)
\end{array}\right) \vec{u}
$$

and

$$
x_{2}^{-2} L=\frac{16-4\left\{\left(\tau_{2} x_{2}^{-2}\right)^{2} \cdot x_{2}^{2} \pm 2\left(\tau_{2} x_{2}^{-2}\right)\right\}\left(4 x_{2}+1\right)}{\left(1 \pm\left(\tau_{2} x_{2}^{-2}\right) \cdot x_{2}^{2}\right)^{2}\left(1+2 x_{2}\right)^{4}}-\frac{\left(\varepsilon x_{2}^{-2}\right)^{2} \cdot x_{2}^{2}}{\left(1+2 x_{2}\right)^{2}}
$$

Hence, the coefficient matrix of (25.2) is reduced to a polynomial of $\left(\varepsilon x_{2}^{-2}\right)$ whose coefficients are functions developable in uniformly convergent double power series of $\left(\tau_{2} x_{2}^{-2}\right)$ and $x_{2}$. Further, since $a_{10}$ and $b_{10}$ (appearing in the domain $\left(\mathfrak{H}_{10}\right)$ ) are supposed to be sufficiently small, the coefficient matrix has two distinct characteristic values in the domain $\left(\mathfrak{S}_{10}\right)$ and they tend to \pm 4 as $\left(\varepsilon x_{2}^{-2}\right),\left(\tau_{2} x_{2}^{-2}\right)$ and $x_{2}$ tend to $00^{-}$This fact shows us that in the domain $\left(\mathfrak{H}_{10}\right)$ the system (25.2) is a system of Type A.

This proves the following:

XI. In the subdomain

(\$夕卜10) $\left\{\begin{array}{l}\left|\varepsilon^{-1} \tau \pm 1\right| \leqq b_{1}, \quad\left|\varepsilon^{-1} x-\frac{1}{2}\right| \leqq c_{2}, \\ \left|\varepsilon\left(\varepsilon^{-1} x-\frac{1}{2}\right)^{-2}\right| \leqq a_{10}, \quad\left|\left(\varepsilon^{-1} \tau \pm 1\right)\left(\varepsilon^{-1} x-\frac{1}{2}\right)^{-2}\right| \leqq b_{10},\end{array}\right.$

a transformation

$$
\left\{\begin{array}{l}
\vec{y}=\left(\begin{array}{cc}
1 & 0 \\
0 & \tau_{1} \varepsilon^{-\frac{1}{2}} \cdot x_{2}
\end{array}\right) \overrightarrow{\mathfrak{w}}, \\
\varepsilon=\alpha, \\
\tau_{2}\left(=\tau_{1} \pm 1=\varepsilon^{-1} \tau \pm 1\right)=\beta, \\
x_{2}\left(=x_{1}-\frac{1}{2}=\varepsilon^{-1} x-\frac{1}{2}\right)=\xi
\end{array}\right.
$$

will reduce the system (W)' to a system of Type A.

\section{$2^{\circ}$. Domain $\left(\mathscr{S}_{11}\right)$.}

Let us put

$$
\left\{\begin{array}{l}
\vec{z}=\left(\begin{array}{cc}
1 & 0 \\
0 & \tau_{2}^{\frac{1}{2}}
\end{array}\right) \vec{u} \\
\varepsilon \tau_{2}^{-1}=\varepsilon_{1} \\
\tau_{2}^{-\frac{1}{2}} x_{2}=x_{3}
\end{array}\right.
$$

Then, we can derive from (24.2) the following:

$$
\varepsilon_{1} \frac{d \vec{u}}{d x_{3}}=\left(\begin{array}{cc}
0 & 1 \\
\tau_{2}^{-1} L & 0
\end{array}\right) \vec{u},
$$

where

$$
\begin{aligned}
\tau_{2}^{-1} L & \equiv \tau_{2}^{-1} L\left(\varepsilon_{1} \tau_{2}, \tau_{2}, \tau_{2}^{\frac{1}{2}} x_{3}\right) \\
& =\frac{16 x_{3}^{2}-16\left(\tau_{2} \pm 2\right) \tau_{2}^{\frac{1}{2}} x_{3}-4\left(\tau_{2} \pm 2\right)}{\left(1 \pm \tau_{2}\right)^{2}\left(1+2 \tau_{2}^{\frac{1}{2}} x_{3}\right)^{4}}-\frac{\varepsilon_{1}^{2} \tau_{2}}{\left(1+2 \tau_{2}^{\frac{1}{2}} x_{3}\right)^{2}}
\end{aligned}
$$


It follows that the coefficient matrix is a polynomial of $\varepsilon_{1}$ whose coefficient are functions developable in uniformly convergent double power series of $\tau_{2}^{\frac{1}{2}}$ and $x_{3}$ for

$$
\left|\varepsilon_{1}\right| \leqq a_{11}, \quad\left|\tau_{2}\right| \leqq b_{1}, \quad\left|x_{3}\right| \leqq c_{11}^{\frac{1}{2}} .
$$

Moreover, the coefficient matrix has two distinct characteristic values in the domain $\left(\widetilde{\mathfrak{H}}_{11}\right)$.

Thus we see that, if we replace $\tau_{2}$ by $\beta^{2}$, the resulting equations are of Type E. Hence we have the following:

XII. In the subdomain

$\left(\mathfrak{S}_{11}\right)$

$$
\left\{\begin{array}{l}
\left|\varepsilon^{-1} \tau \pm 1\right| \leqq b_{1}, \quad\left|\varepsilon^{-1} x-\frac{1}{2}\right| \leqq c_{2}, \\
\left|\varepsilon\left(\varepsilon^{-1} \tau \pm 1\right)^{-1}\right| \leqq a_{11}, \quad\left|\left(\varepsilon^{-1} \tau \pm 1\right)^{-1}\left(\varepsilon^{-1} x-\frac{1}{2}\right)^{2}\right| \leqq c_{11},
\end{array}\right.
$$

the equations (W)' will be reduced to a system of Type $\mathrm{E}$ if we put

$$
\left\{\begin{array}{l}
\vec{y}=\left(\begin{array}{cc}
1 & 0 \\
0 & \tau_{1} \varepsilon^{-\frac{1}{2}} \cdot \tau_{2}^{\frac{1}{2}}
\end{array}\right) \overrightarrow{\mathfrak{w}}, \\
\varepsilon_{1}\left(=\varepsilon \tau_{2}^{-1}=\varepsilon\left(\varepsilon^{-1} \tau \pm 1\right)^{-1}\right)=\alpha, \\
\tau_{2}\left(=\varepsilon^{-1} \tau \pm 1\right)=\beta^{2}, \\
x_{3}\left(=\tau_{2}^{-\frac{1}{2}} x_{2}=\left(\varepsilon^{-1} \tau \pm 1\right)^{-\frac{1}{2}}\left(\varepsilon^{-1} x-\frac{1}{2}\right)\right)=\xi .
\end{array}\right.
$$

$3^{\circ}$. Domain $\left(\mathscr{S}_{12}^{\prime}\right)$.

If we make a change of variables:

$$
\left\{\begin{array}{l}
\vec{z}=\left(\begin{array}{ll}
1 & 0 \\
0 & \varepsilon^{\frac{1}{2}}
\end{array}\right) \vec{u} \\
\varepsilon^{-1} \tau_{2}=\tau_{3} \\
\varepsilon^{-\frac{1}{2}} x_{2}=x_{3}
\end{array}\right.
$$

the equations (24.2) will be transformed into a system

$$
\frac{d \vec{u}}{d x_{3}}=\left(\begin{array}{cc}
0 & 1 \\
\varepsilon^{-1} L & 0
\end{array}\right) \vec{u}
$$

with

$$
\varepsilon^{-1} L=\frac{16 x_{3}^{2}-4\left(\varepsilon \tau_{3}^{2} \pm 2 \tau_{3}\right)\left(4 \varepsilon^{\frac{1}{2}} x_{3}+1\right)}{\left(1 \pm \varepsilon \tau_{3}\right)^{2}\left(1+2 \varepsilon^{\frac{1}{2}} x_{3}\right)^{4}}-\frac{\varepsilon}{\left(1+2 \varepsilon^{\frac{1}{2}} x_{3}\right)^{2}} .
$$

Since the quantity $a_{0}$ is supposed to be sufficiently small, we can suppose the following inequalities to be satisfied:

$$
\left(\left|\varepsilon \tau_{3}\right| \leqq\right) a_{0} B_{12}<1, \quad\left(\left|\varepsilon^{\frac{1}{2}} x_{3}\right| \leqq\right) a_{0}^{\frac{1}{2}} C_{12^{\frac{1}{2}}}<1 .
$$

Therefore, in the domain $\left(\mathfrak{S}_{12}^{\prime}\right)$ the function $\varepsilon^{-1} L$ is developable in a uniformly convergent double power series of $\varepsilon^{\frac{1}{2}}$ and $\tau_{3}$ whose coefficients are polynomials of $x_{3}$.

If we put further $\varepsilon=\alpha^{2}$, the system (25.6) becomes a system of Type F. 
Hence, we have the following:

\section{In the subdomain}

$\left(\mathfrak{S}_{12}^{\prime}\right)$

$$
\left\{\begin{array}{l}
\left|\varepsilon^{-1} \tau \pm 1\right| \leqq b_{1}, \quad\left|\varepsilon^{-1} x-\frac{1}{2}\right|^{2} \leqq c_{2}, \\
\left|\varepsilon^{-1}\left(\varepsilon^{-1} \tau \pm 1\right)\right| \leqq B_{12}, \quad\left|\varepsilon^{-1}\left(\varepsilon^{-1} x-\frac{1}{2}\right)^{2}\right| \leqq C_{12},
\end{array}\right.
$$

the system (WM)' will be reduced to a system of Type $\mathrm{F}$ by a transformation

$$
\left\{\begin{array}{l}
\vec{y}=\left(\begin{array}{lc}
1 & 0 \\
0 & \tau_{1} \varepsilon^{-\frac{1}{2}} \cdot \varepsilon^{\frac{1}{2}}
\end{array}\right) \overrightarrow{\mathfrak{w}}, \\
\varepsilon=\alpha^{2}, \\
\tau_{3}\left(=\varepsilon^{-1} \tau_{2}=\varepsilon^{-1}\left(\varepsilon^{-1} \tau \pm 1\right)\right)=\beta, \\
x_{3}\left(=\varepsilon^{-\frac{1}{2}} x_{2}=\varepsilon^{-\frac{1}{2}}\left(x_{1}-\frac{1}{2}\right)=\varepsilon^{-\frac{1}{2}}\left(\varepsilon^{-1} x-\frac{1}{2}\right)\right)=\xi .
\end{array}\right.
$$

\section{§26. Domain $\left(\mathfrak{S}_{13}\right)$.}

In order to study the behavior of solutions of the system (24.2) in the subdomain

$$
c_{13} \leqq\left|\tau_{2}^{-\frac{1}{2}} x_{2}\right| \leqq C_{13},
$$

we introduce a new independent variable $x_{3}$ :

$$
x_{3}=\tau_{2}^{-\frac{1}{2}} x_{2} .
$$

Then, the system (24.2) will be reduced to

$$
\varepsilon \tau_{2}^{-\frac{1}{2}} \frac{d \vec{z}}{d x_{3}}=\left(\begin{array}{cc}
0 & 1 \\
M\left(\varepsilon, \tau_{2}, x_{3}\right) & 0
\end{array}\right) \vec{z}
$$

where

$$
M=\frac{16 \tau_{2} x_{3}^{2}-16\left(\tau_{2}^{2} \pm 2 \tau_{2}\right) \tau_{2}^{\frac{1}{2}} x_{3}-4\left(\tau_{2}^{2} \pm 2 \tau_{2}\right)}{\left(1 \pm \tau_{2}\right)^{2}\left(1+2 \tau_{2}^{\frac{1}{2}} x_{3}\right)^{4}}-\frac{\varepsilon^{2}}{\left(1+2 \tau_{2}^{\frac{1}{2}} x_{3}\right)^{2}} .
$$

Therefore, the system (26.2) has no singularity with respect to $x_{3}$ in the considered domain

$$
0<|\varepsilon| \leqq a_{0}, \quad\left|\tau_{2}\right| \leqq b_{1}, \quad c_{13} \leqq\left|x_{3}\right| \leqq C_{13} .
$$

Let us construct a characteristic polygon of (26.2) with respect to $\varepsilon$ and $\tau_{2}$. Then, we see easily that the characteristic polygon has two vertices:

$$
\mathrm{Q}_{0}=\left(0, \frac{1}{2}\right), \quad \mathrm{Q}_{1}=\left(1,-\frac{1}{2}\right)
$$

as is shown in figure.

We divide the domain $\left(\widetilde{\mathfrak{H}}_{13}\right)$ into following two subdomains :

$\begin{array}{ll}\left(\mathrm{H}_{14}\right) & \left|\varepsilon \tau_{2}^{-1}\right| \leqq a_{14} ; \\ \left(\mathrm{H}_{15}\right) & \left|\varepsilon^{-1} \tau_{2}\right| \leqq B_{15} \quad\left(B_{15}=1 / a_{14}\right) .\end{array}$

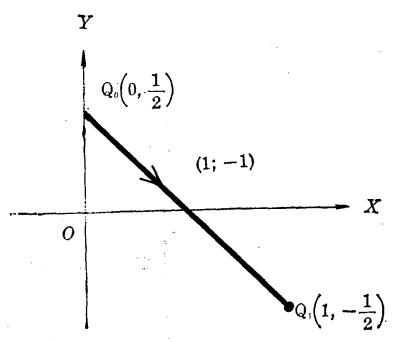

Fig. 11 
§ 27. Domain $\left(\mathbf{H}_{15}\right)$.

We consider the subdomain $\left(\mathrm{H}_{15}\right)$. Noticing that the coordinate of the vertex $\mathrm{Q}_{1}$ is $\left(1,-\frac{1}{2}\right)$, let us make a change of variables :

$$
\left\{\begin{array}{l}
\vec{z}=\left(\begin{array}{cc}
1 & 0 \\
0 & \varepsilon \tau_{2}^{-\frac{1}{2}}
\end{array}\right) \vec{u}, \\
\varepsilon^{-1} \tau_{2}=\tau_{3} .
\end{array}\right.
$$

Then, we can derive from (26.2) the following:

$$
\frac{d \vec{u}}{d x_{3}}=\left(\begin{array}{cc}
0 & 1 \\
\varepsilon^{-2} \tau_{2} M\left(\varepsilon, \varepsilon \tau_{3}, x_{3}\right) & 0
\end{array}\right) \vec{u}
$$

and the domain $\left(\mathrm{H}_{15}\right)$ is reduced to

$\left(\tilde{\mathrm{H}}_{15}\right) \quad 0<|\varepsilon| \leqq a_{0}, \quad\left|\tau_{3}\right| \leqq B_{15}, \quad c_{13} \leqq\left|x_{3}\right| \leqq C_{13}$,

where, in new variables,

Clearly,

$$
\varepsilon^{-2} \tau_{2} M=\frac{16 \tau_{3}^{2} x_{3}^{2}-16\left(\varepsilon \tau_{3}^{3} \pm 2 \tau_{3}^{2}\right) \varepsilon^{\frac{1}{2}} \tau_{3}^{\frac{1}{2}} x_{3}-4\left(\varepsilon \tau_{3}^{3} \pm 2 \tau_{3}^{2}\right)}{\left(1 \pm \varepsilon \tau_{3}\right)^{2}\left(1+2 \varepsilon^{\frac{1}{2}} \tau_{3}^{\frac{1}{2}} x_{3}\right)^{4}}-\frac{\varepsilon \tau_{3}}{\left(1+2 \varepsilon^{\frac{1}{2}} \tau_{3}^{\frac{1}{2}} x_{3}\right)^{2}} .
$$

$$
\left|\varepsilon \tau_{3}\right| \leqq a_{0} B_{15}, \quad\left|\varepsilon^{\frac{1}{2}} \tau_{3}^{\frac{1}{2}} x_{3}\right| \leqq a_{0}^{\frac{1}{2}} B_{15} \frac{\frac{1}{2}}{2} C_{13} .
$$

However, since the quantity $a_{0}$ is supposed to be sufficiently small, we can suppose that the following inequalities are satisfied:

$$
a_{0} B_{15}<1, \quad 2 a_{0}^{\frac{1}{2}} B_{15}{ }^{\frac{1}{2}} C_{13}<1 .
$$

This assures us that the function $\varepsilon^{-2} \tau_{2} M$ can be expanded in a uniformly convergent power series of $\varepsilon^{\frac{1}{2}}$ :

$$
\varepsilon^{-2} \tau_{2} M=\sum_{h=0}^{\infty} \varepsilon^{\frac{h}{2}} M_{h}\left(\tau_{3}, x_{3}\right),
$$

valid in the domain $\left(\widetilde{\mathrm{H}}_{15}\right)$, where $M_{h}\left(\tau_{3}, x_{3}\right)$ are polynomials of $\tau_{3}^{\frac{1}{2}}$ and $x_{3}$.

Thus we see that, by substituting $\varepsilon$ and $\tau_{3}$ by $\alpha^{2}$ and $\beta^{2}$ respectively, the system (27.2) is reduced to a system of Type F.

Hence we have the following:

XIV. In the subdomain

$\left(\mathrm{H}_{15}\right) \quad\left\{\begin{array}{l}\left|\varepsilon^{-1} \tau \pm 1\right| \leqq b_{1}, \quad\left|\varepsilon^{-1} x-\frac{1}{2}\right| \leqq c_{2}, \\ \left|\varepsilon^{-1}\left(\varepsilon^{-1} \tau \pm 1\right)\right| \leqq B_{15}, \quad c_{13} \leqq\left|\left(\varepsilon^{-1} \tau \pm 1\right)^{-\frac{1}{2}}\left(\varepsilon^{-1} x-\frac{1}{2}\right)\right| \leqq C_{13},\end{array}\right.$

\section{a transformation}

$$
\left\{\begin{array}{l}
\vec{y}=\left(\begin{array}{lc}
1 & 0 \\
0 & \tau_{1} \varepsilon^{-\frac{1}{2}} \cdot \varepsilon \tau_{2}^{-\frac{1}{2}}
\end{array}\right) \overrightarrow{\mathfrak{w}}, \\
\varepsilon=\alpha^{2}, \\
\tau_{3}\left(=\varepsilon^{-1} \tau_{2}=\varepsilon^{-1}\left(\varepsilon^{-1} \tau \pm 1\right)\right)=\beta^{2}, \\
x_{3}\left(=\tau_{2}^{-\frac{1}{2}} x_{2}=\tau_{2}^{-\frac{1}{2}}\left(x_{1}-\frac{1}{2}\right)=\left(\varepsilon^{-1} \tau \pm 1\right)^{-\frac{1}{2}}\left(\varepsilon^{-1} x-\frac{1}{2}\right)\right)=\xi
\end{array}\right.
$$


will reduce the system (W)' to a system of Type $\mathrm{F}$.

\section{§ 28. Domain $\left(\mathbf{H}_{14}\right)$.}

We consider again the system (26.2). Noticing that the coordinate of the vertex $Q_{0}$ is $\left(0, \frac{1}{2}\right)$, put

$$
\left\{\begin{array}{l}
\vec{z}=\left(\begin{array}{cc}
1 & 0 \\
0 & \tau_{2}^{\frac{1}{2}}
\end{array}\right) \vec{u}, \\
\varepsilon \tau_{2}^{-1}=\varepsilon_{1} .
\end{array}\right.
$$

Then, the system (26.2) will be changed into a system

$$
\varepsilon_{1} \frac{d \vec{u}}{d x_{3}}=\left(\begin{array}{cc}
0 & 1 \\
\tau_{2}^{-1} M\left(\varepsilon_{1} \tau_{2}, \tau_{2}, x_{3}\right) & 0
\end{array}\right) \vec{u}
$$

and the domain $\left(\mathrm{H}_{14}\right)$ becomes

$$
\left(\widetilde{\mathrm{H}}_{14}\right) \quad\left|\varepsilon_{1}\right| \leqq a_{14}, \quad\left|\tau_{2}\right| \leqq b_{1}, \quad c_{13} \leqq\left|x_{3}\right| \leqq C_{13} .
$$

The expression (26.3) yields :

$$
\tau_{2}^{-1} M=\frac{16 x_{3}^{2}-16\left(\tau_{2} \pm 2\right) \tau_{2}^{\frac{1}{2}} x_{3}-4\left(\tau_{2} \pm 2\right)}{\left(1 \pm \tau_{2}\right)^{2}\left(1+2 \tau_{2}^{\frac{1}{2}} x_{3}\right)^{4}}-\frac{\varepsilon_{1}^{2} \tau_{2}}{\left(1+2 \tau_{2}^{\frac{1}{2}} x_{3}\right)^{2}} .
$$

Therefore, the system (28.2) has no singularity with respect to $x_{3}$. Consequently, we may take $c_{13}=0$ in $\left(\tilde{\mathrm{H}}_{14}\right)$. Clearly, $\left|\tau_{2}^{\frac{1}{2}} x_{3}\right| \leqq b_{1}^{\frac{1}{2}} C_{13}$. Since $b_{1}$ is supposed to be sufficiently small, we may assume the inequality $2 b_{1}^{\frac{1}{2}} C_{13}<1$. Therefore, the function $\tau_{2}^{-1} M$ is a polynomial of $\varepsilon_{1}$ and its coefficients are functions which can be developable in uniformly convergent power series of $\tau_{2}^{\frac{1}{2}}$ whose coefficients are polynomials of $x_{3}$.

On the other hand, we see immediately that the system (28.2) has only two turning points at

$$
x_{3}= \pm \frac{1}{\sqrt{2}}+O\left(\tau_{2}^{\frac{1}{2}}\right) \quad \text { or } \pm \frac{i}{\sqrt{2}}+O\left(\tau_{2}^{\frac{1}{2}}\right)
$$

Indeed, such turning points are two roots of the following algebraic equation:

$$
4 x_{3}^{2}-4\left(\tau_{2}+2\right) \tau_{2} \frac{1}{2} x_{3}-\left(\tau_{2}+2\right)=0
$$

or

$$
4 x_{3}^{2}-4\left(\tau_{2}-2\right) \tau_{2}^{\frac{1}{2}} x_{3}-\left(\tau_{2}-2\right)=0 .
$$

The two roots are given by

$$
x_{3}=g_{ \pm}\left(\tau_{2}\right) \text {, }
$$

where

$$
g_{ \pm}\left(\tau_{2}\right)=\left\{\begin{array}{l}
\frac{1}{2}\left[\left(\tau_{2}+2\right) \tau_{2}^{\frac{1}{2}} \pm \sqrt{\tau_{2}^{3}+4 \tau_{2}^{2}+5 \tau_{2}+2}\right] \\
\frac{1}{2}\left[\left(\tau_{2}-2\right) \tau_{2}^{\frac{1}{2}} \pm \sqrt{\tau_{2}^{3}-4 \tau_{2}^{2}+5 \tau_{2}-2}\right]
\end{array}\right.
$$

Since $b_{1}$ is supposed to be sufficiently small, we can suppose 
for $\left|\tau_{2}\right| \leqq b_{1}$ and the functions $g_{ \pm}\left(\tau_{2}\right)$ can be expanded in a uniformly convergent power series of $\tau_{2}^{\frac{1}{2}}$. Clearly, if $x_{3} \neq g_{ \pm}\left(\tau_{2}\right)$, the coefficient matrix of the system (28.2) has two distinct characteristic values in the domain $\left(\tilde{\mathrm{H}}_{14}\right)$.

We divide the domain $\left(\widetilde{\mathrm{H}}_{14}\right)$ into following two subdomains :

$$
\begin{aligned}
& \left|x_{3}-g_{ \pm}\left(\tau_{2}\right)\right| \geqq c_{3} ; \\
& \left|x_{3}-g_{ \pm}\left(\tau_{2}\right)\right| \leqq c_{3} .
\end{aligned}
$$

Then, it follows immediately that, in the domain $\left(\mathrm{H}_{16}\right)$, the system (28.2) (where we put $\varepsilon_{1}=\alpha, \tau_{2}=\beta^{2}, x_{3}=\xi$ ) is a system of Type E.

Thus we have the following:

XV. In the subdomain

$\left(\mathrm{H}_{16}\right) \quad\left\{\begin{array}{l}\left|\varepsilon^{-1} \tau \pm 1\right| \leqq b_{1}, \quad\left|\varepsilon^{-1} x-\frac{1}{2}\right| \leqq c_{2}, \\ \left|\varepsilon\left(\varepsilon^{-1} \tau \pm 1\right)^{-1}\right| \leqq a_{14}, \quad c_{13} \leqq\left|\left(\varepsilon^{-1} \tau \pm 1\right)^{-\frac{1}{2}}\left(\varepsilon^{-1} x-\frac{1}{2}\right)\right| \leqq C_{13}, \\ \left|\left(\varepsilon^{-1} \tau \pm 1\right)^{-\frac{1}{2}}\left(\varepsilon^{-1} x-\frac{1}{2}\right)-g_{ \pm}\left(\varepsilon^{-1} \tau \pm 1\right)\right| \geqq c_{3},\end{array}\right.$

a transformation

$$
\left\{\begin{array}{l}
\vec{y}=\left(\begin{array}{cc}
1 & 0 \\
0 & \tau_{1} \varepsilon^{-\frac{1}{2}} \cdot \tau_{2} \frac{1}{2}
\end{array}\right) \overrightarrow{\mathfrak{w}}, \\
\varepsilon_{1}\left(=\varepsilon \tau_{2}^{-1}=\varepsilon\left(\varepsilon^{-1} \tau \pm 1\right)^{-1}\right)=\alpha, \\
\tau_{2}\left(=\varepsilon^{-1} \tau \pm 1\right)=\beta^{2}, \\
x_{3}\left(=\tau_{2}^{-\frac{1}{2}} x_{2}=\tau_{2}^{-\frac{1}{2}}\left(\varepsilon^{-1} x-\frac{1}{2}\right)=\left(\varepsilon^{-} \tau \pm 1\right)^{-\frac{1}{2}}\left(\varepsilon^{-1} x-\frac{1}{2}\right)\right)=\xi
\end{array}\right.
$$

will reduce the system (W) to a system of Type $\mathrm{E}$.

§29. Domain $\left(\mathbf{H}_{17}\right)$.

\section{$1^{\circ}$. Characteristic polyhedron.}

In order to study the behavior of solutions of the system (28.2) in the domain $\left(\mathrm{H}_{17}\right)$ :

$$
\left|\varepsilon_{1}\right| \leqq a_{14}, \quad\left|\tau_{2}\right| \leqq b_{1}, \quad\left|x_{3}-g_{ \pm}\left(\tau_{2}\right)\right| \leqq c_{3},
$$

we introduce a new independent variable

$$
x_{4}=x_{3}-g_{ \pm}\left(\tau_{2}\right)
$$

so that the domain $\left(\mathrm{H}_{17}\right)$ becomes

$\left(\widetilde{\mathrm{H}}_{17}\right)$

$$
\left|\varepsilon_{1}\right| \leqq a_{14}, \quad\left|\tau_{2}\right| \leqq b_{1}, \quad\left|x_{4}\right| \leqq c_{3} .
$$

The considered system is reduced to

$$
\varepsilon_{1} \frac{d \vec{u}}{d x_{4}}=\left(\begin{array}{cc}
0 & 1 \\
N\left(\varepsilon_{1}, \tau_{2}, x_{4}\right) & 0
\end{array}\right) \vec{u},
$$


where

$$
N=\frac{16 x_{4}^{2}+16\left[2 g_{ \pm}\left(\tau_{2}\right)-\left(\tau_{2} \pm 2\right) \tau_{2}^{\frac{1}{2}}\right] x_{4}}{\left(1 \pm \tau_{2}\right)^{2}\left(1+2 \tau_{2}^{\frac{1}{2}} g_{ \pm}\left(\tau_{2}\right)+2 \tau_{2}^{\frac{1}{2}} x_{4}\right)^{4}}-\frac{\varepsilon_{1}^{2} \tau_{2}}{\left(1+2 \tau_{2}^{\frac{1}{2}} g_{ \pm}+2 \tau_{2}^{\frac{1}{2}} x_{4}\right)^{2}} .
$$

Hence, the coefficient matrix of (29.2) is developable in a uniformly convergent triple power series of $\varepsilon_{1}, \tau_{2}^{\frac{1}{2}}$ and $x_{4}$. Further, we see from (28.3) that the expression $2 g_{ \pm}\left(\tau_{2}\right)-\left(\tau_{2} \pm 2\right) \tau_{2}^{\frac{1}{2}}$ does not vanish at $\tau_{2}=0$.

Now let us construct a characteristic polyhedron of (29.2). However, since the point $x_{4}=0$ is a regular point of the considered system, it is sufficient to consider a modified characteristic polyhedron. As is shown in figure, the modified characteristic polyhedron has two vertices :

$$
\mathrm{V}_{1}=\left(0,0, \frac{1}{2}\right), \quad \mathrm{V}_{2}=\left(\frac{1}{3}, 0,0\right)
$$

and is regarded as a trigonal cylinder with its base on $(X, Z)$-plane.

$2^{\circ}$. Division of the domain $\left(\widetilde{\mathbf{H}}_{17}\right)$.

It is easily seen that at each vertex

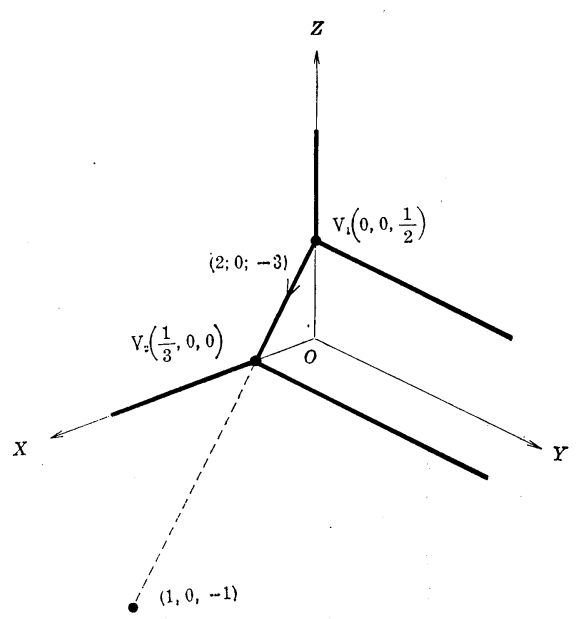

Fig. 12 there exists only one characteristic trigonal pyramid. The principal subdomains are given by

$$
\left|\varepsilon_{1} x_{4}^{-\frac{3}{2}}\right| \leqq a_{18} ; \quad\left|\varepsilon_{1}^{-\frac{2}{3}} x_{4}\right| \leqq c_{19} .
$$

However, we can give $c_{19}$ a value as large as we want. Hence, we put $c_{19}=$ $a_{18}^{-\frac{2}{3}}\left(\equiv C_{19}\right)$.

Clearly, the union of these two subdomains covers the domain $\left(\widetilde{\mathrm{H}}_{17}\right)$ without lacuna.

\section{$\S 30$. Domain $\left(\mathfrak{S}_{18}\right)$.}

Noticing that the coordinate of the vertex $\mathrm{V}_{1}$ is $\left(0,0, \frac{1}{2}\right)$, put

$$
\vec{u}=\left(\begin{array}{cc}
1 & 0 \\
0 & x_{4^{\frac{1}{2}}}
\end{array}\right) \vec{v}
$$

Then the system (29.2) will be transformed into a system

$$
\left(\varepsilon_{1} x_{4}^{-\frac{3}{2}}\right) \cdot x_{4} \frac{d \vec{v}}{d x_{4}}=\left(\begin{array}{cc}
0 & 1 \\
x_{4}^{-1} N & -\frac{1}{2}\left(\varepsilon_{1} x_{4}^{-\frac{3}{2}}\right)
\end{array}\right) \vec{v},
$$

where

$$
x_{4}^{-1} N=\frac{16 x_{4}+16\left(2 g_{ \pm}\left(\tau_{2}\right)-\left(\tau_{2} \pm 2\right) \tau_{2}^{\frac{1}{2}}\right)}{\left(1 \pm \tau_{2}\right)^{2}\left(1+2 \tau_{2}^{\frac{1}{2}} g_{ \pm}\left(\tau_{2}\right)+2 \tau_{2}^{\frac{1}{2}} x_{4}\right)^{4}}-\frac{\left(\varepsilon_{1} x_{4}^{-\frac{3}{2}}\right)^{2} \cdot \tau_{2} \cdot x_{4}^{2}}{\left(1+2 \tau_{2}^{\frac{1}{2}} g_{ \pm}+2 \tau_{2}^{\frac{1}{2}} x_{4}\right)^{2}}
$$


Hence, the coefficient matrix is a polynomial of $\left(\varepsilon_{1} x_{4}^{-\frac{3}{2}}\right)$ whose coefficients are functions admitting uniformly convergent expansions in powers of $\tau_{2}^{\frac{1}{2}}$ in the domain $\left(\mathfrak{S}_{18}\right)$ (whose coefficients are polynomials of $x_{4}$ ). As $\varepsilon_{1} x_{4}^{-\frac{3}{2}}, \tau_{2}$ and $x_{4}$ tend to 0 , the coefficient matrix will be reduced to a matrix

$$
\left(\begin{array}{cc}
0 & 1 \\
32 g_{ \pm}(0) & 0
\end{array}\right)
$$

Since, as we have already seen (cf. (28.3)),

$$
g_{ \pm}(0)= \pm \frac{1}{\sqrt{2}} \text { or } \pm \frac{i}{\sqrt{2}}
$$

this matrix has two distinct characteristic values. Consequently, in the domain $\left(\mathfrak{S}_{18}\right)$ the system (30.2) (where we have to put $\varepsilon_{1}=\alpha, \tau_{2}=\beta^{2}, x_{4}=\xi$ ) is a system of Type C.

Thus we have the following:

XVI. In the subdomain

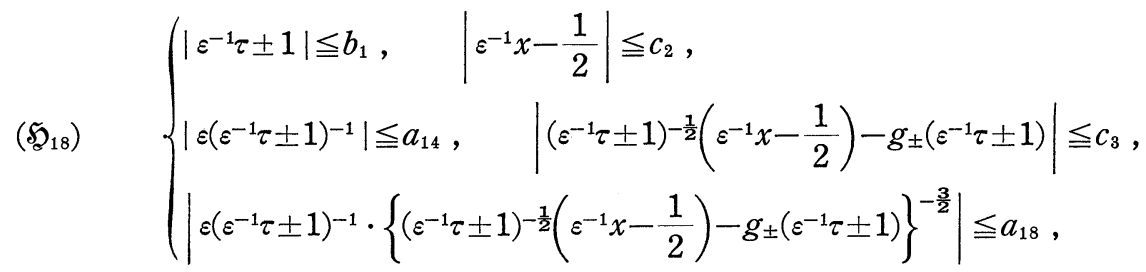

a transformation

$$
\left\{\begin{array}{l}
\vec{y}=\left(\begin{array}{ll}
1 & 0 \\
0 & \tau_{1} \varepsilon^{-\frac{1}{2}} \cdot \tau_{2}^{\frac{1}{2}} \cdot x_{4}^{\frac{1}{2}}
\end{array}\right) \overrightarrow{\mathrm{w}}, \\
\varepsilon_{1}\left(=\varepsilon \tau_{2}^{-1}=\varepsilon\left(\varepsilon^{-1} \tau \pm 1\right)^{-1}\right)=\alpha \\
\tau_{2}\left(=\varepsilon^{-1} \tau \pm 1\right)=\beta^{2}, \\
x_{4}\left(=x_{3}-g_{ \pm}\left(\tau_{2}\right)=\tau_{2}^{-\frac{1}{2}} x_{2}-g_{ \pm}=\left(\varepsilon^{-1} \tau \pm 1\right)^{-\frac{1}{2}}\left(\varepsilon^{-1} x-\frac{1}{2}\right)-g_{ \pm}\right)=\xi
\end{array}\right.
$$

will change the system (W)' into a system of Type $\mathrm{C}$.

\section{§ 31. Domain $\left(\mathfrak{S}_{19}\right)$.}

We consider the system (29.2). Make a change of variables :

$$
\left\{\begin{array}{l}
\vec{u}=\left(\begin{array}{cc}
1 & 0 \\
0 & \varepsilon_{1}^{\frac{1}{3}}
\end{array}\right) \vec{v}, \\
\varepsilon_{1}^{-\frac{2}{3}} x_{4}=x_{5},
\end{array}\right.
$$

noticing that the coordinate of the vertex $\mathrm{V}_{2}$ is $\left(\frac{1}{3}, 0,0\right)$, then the system (29.2) is transformed into a system

$$
\frac{d \vec{v}}{d x_{5}}=\left(\begin{array}{cc}
0 & 1 \\
\varepsilon_{1}^{-\frac{2}{3}} N & 0
\end{array}\right) \vec{v},
$$

where 


$$
\varepsilon_{1}^{-\frac{2}{3}} N=\frac{16 \varepsilon_{1}^{\frac{2}{3}} x_{5}^{2}+16\left[2 g_{ \pm}\left(\tau_{2}\right)-\left(\tau_{2} \pm 2\right) \tau_{2}^{\frac{1}{2}}\right] x_{5}}{\left(1 \pm \tau_{2}\right)^{2}\left(1+2 \tau_{2}^{\frac{1}{2}} g_{ \pm}\left(\tau_{2}\right)+2 \varepsilon_{1}^{\frac{2}{3}} \tau_{2}^{\frac{1}{2}} x_{5}\right)^{4}}-\frac{\varepsilon_{1}^{\frac{4}{3}} \tau_{2}}{\left(1+2 \tau_{2}^{\frac{1}{2}} g_{ \pm}+2 \varepsilon_{1}{ }^{\frac{2}{3}} \tau_{2}^{\frac{1}{2}} x_{5}\right)^{2}}
$$

The domain $\left(\mathfrak{S}_{19}\right)$ is reduced to

$$
\left|\varepsilon_{1}\right| \leqq a_{14}, \quad\left|\tau_{2}\right| \leqq b_{1}, \quad\left|x_{5}\right| \leqq C_{19} .
$$

Hence

$$
\left|\varepsilon_{1}^{\frac{2}{3}} \tau_{2}^{\frac{1}{2}} x_{5}\right| \leqq a_{14^{\frac{2}{3}}} b_{1}^{\frac{1}{2}} C_{19}, \quad\left|\tau_{2}^{\frac{1}{2}} g_{ \pm}\left(\tau_{2}\right)\right| \leqq b_{1}^{\frac{1}{2}} C_{13}
$$

(See (28.4)). Therefore the expression $\varepsilon_{1}^{-\frac{2}{3}} N$ can be expanded in a uniformly convergent double power series of $\varepsilon_{1}^{\frac{1}{3}}$ and $\tau_{2}^{\frac{1}{2}}$ whose coefficients are polynomials of $x_{5}$ provided that, no matter how $C_{13}, C_{19}$ are large, the quantity $b_{1}$ is sufficiently small.

Hence if we substitute $\varepsilon_{1}$ and $\tau_{2}$ by $\alpha^{3}$ and $\beta^{2}$ respectively, the system (31.2) becomes a system of Type F.

Thus we have the following:

XVII. In the subdomain

$$
\left\{\begin{array}{l}
\left|\varepsilon^{-1} \tau \pm 1\right| \leqq b_{1}, \quad\left|\varepsilon^{-1} x-\frac{1}{2}\right| \leqq c_{2}, \\
\left|\varepsilon\left(\varepsilon^{-1} \tau \pm 1\right)^{-1}\right| \leqq a_{14}, \quad\left|\left(\varepsilon^{-1} \tau \pm 1\right)^{-\frac{1}{2}}\left(\varepsilon^{-1} x-\frac{1}{2}\right)-g_{ \pm}\left(\varepsilon^{-1} \tau \pm 1\right)\right| \leqq c_{3}, \\
\left|\left\{\varepsilon\left(\varepsilon^{-1} \tau \pm 1\right)^{-1}\right\}^{-\frac{2}{3}}\left\{\left(\varepsilon^{-1} \tau \pm 1\right)^{-\frac{1}{2}}\left(\varepsilon^{-1} x-\frac{1}{2}\right)-g_{ \pm}\left(\varepsilon^{-1} \tau \pm 1\right)\right\}\right| \leqq C_{19},
\end{array}\right.
$$

the system (W)' will be reduced to a system of Type $\mathrm{F}$ by a transformation

$$
\left\{\begin{array}{l}
\vec{y}=\left(\begin{array}{ll}
1 & 0 \\
0 & \tau_{1} \varepsilon^{-\frac{1}{2}} \cdot \tau_{2}^{\frac{1}{2}} \cdot \varepsilon_{1}^{\frac{1}{3}}
\end{array}\right) \overrightarrow{\mathfrak{w}}, \\
\varepsilon_{1}\left(=\varepsilon \tau_{2}^{-1}=\varepsilon\left(\varepsilon^{-1} \tau \pm 1\right)^{-1}\right)=\alpha^{3}, \\
\tau_{2}\left(=\varepsilon^{-1} \tau \pm 1\right)=\beta^{2}, \\
x_{5}\left(=\varepsilon_{1}^{-\frac{2}{3}} x_{4}=\left\{\varepsilon\left(\varepsilon^{-1} \tau \pm 1\right)^{-1}\right\}^{-\frac{2}{3}}\left\{\left(\varepsilon^{-1} \tau \pm 1\right)^{-\frac{1}{2}}\left(\varepsilon^{-1} x-\frac{1}{2}\right)-g_{ \pm}\right\}\right)=\xi .
\end{array}\right.
$$

\section{§32. Conclusion.}

By the propositions obtained in this chapter, we can easily construct, by the help of fundamental lemmas in Chapter IX, an asymptotic expression of a fundamental set of independent solutions of Whittaker's differential equation (W) as $\varepsilon, \tau$ and $x$ tend to 0 under the restriction such that

$$
b \leqq\left|\varepsilon^{-1} \tau\right| \leqq B .
$$

Summarizing the above discussions, we can state the following:

Theorem 1. In order to investigate the behavior of solutions of the Whittaker's differential equation (W) in the domain

$\left(\mathrm{D}_{7}\right) \quad b \leqq\left|\varepsilon^{-1} \tau\right| \leqq B, \quad 0<|\varepsilon| \leqq a_{0}, \quad 0<|\tau| \leqq b_{0}, \quad 0<|x| \leqq c_{0}$,

it is sufficient to divide the domain $\left(\mathrm{D}_{7}\right)$ into twelve subdomains. Such subdomains 
and the types of normal form in each of subdomains are given by a following table:

\begin{tabular}{l|c|c|c|c|c|c|c|c|c|c|c|c}
\hline Subdomain & $\mathrm{H}_{1}$ & $\mathrm{H}_{2}$ & $\mathrm{H}_{4}$ & $\mathrm{H}_{6}$ & $\mathrm{H}_{8}$ & $\mathfrak{S}_{10}$ & $\mathfrak{S}_{11}$ & $\mathfrak{S}_{12}^{\prime}$ & $\mathrm{H}_{15}$ & $\mathrm{H}_{16}$ & $\mathfrak{F}_{18}$ & $\mathfrak{F}_{19}$ \\
\hline Normal form & $\mathrm{D}$ & $\mathrm{C}$ & $\mathrm{E}$ & $\mathrm{F}$ & $\mathrm{C}$ & $\mathrm{A}$ & $\mathrm{E}$ & $\mathrm{F}$ & $\mathrm{F}$ & $\mathrm{E}$ & $\mathrm{C}$ & $\mathrm{F}$ \\
\hline Proposition & $\mathrm{VI}$ & $\mathrm{VII}$ & $\mathrm{VIII}$ & $\mathrm{IX}$ & $\mathrm{X}$ & $\mathrm{XI}$ & $\mathrm{XII}$ & $\mathrm{XIII}$ & $\mathrm{XIV}$ & $\mathrm{XV}$ & $\mathrm{XVI}$ & $\mathrm{XVII}$ \\
\hline Section & 18 & 19 & 20 & 22 & 23 & 25 & 25 & 25 & 27 & 28 & 30 & 31 \\
\hline
\end{tabular}

These twelve subdomains are related in a following way:

$$
\begin{aligned}
& \mathrm{D}_{7}=\mathrm{H}_{1} \cup \mathrm{H}_{2} \cup \mathrm{H}_{3}, \\
& \mathrm{H}_{3}=\mathrm{H}_{4} \cup \mathrm{H}_{5}, \\
& \mathrm{H}_{5}=\mathrm{H}_{6} \cup \mathrm{H}_{7}, \\
& \mathrm{H}_{7}=\mathrm{H}_{8} \cup \mathrm{H}_{9}, \\
& \mathrm{H}_{9} \subset \mathfrak{S}_{10} \cup \mathfrak{S}_{11} \cup \mathfrak{S}_{12}^{\prime} \cup \mathfrak{S}_{13}, \\
& \mathfrak{S}_{13}=\mathrm{H}_{14} \cup \mathrm{H}_{15}, \\
& \mathrm{H}_{14}=\mathrm{H}_{16} \cup \mathrm{H}_{17}, \\
& \mathrm{H}_{17}=\mathfrak{S}_{18} \cup \mathfrak{S}_{19},
\end{aligned}
$$

\section{Chapter V. Study of the behavior of the solutions in the auxiliary subdomain $\left(D_{8}\right)$.}

In this chapter, we will study the behavior of solutions of the system (WB) in an auxiliary subdomain of the form

$$
\left|\varepsilon \tau^{-1}\right| \leqq a, \quad c \leqq\left|\varepsilon^{-1} x\right| \leqq C .
$$

We shall see here that the reduced forms of the system (WB) are of Type A, $\mathrm{C}, \mathrm{E}, \mathrm{F}$ and of the following new type:

$$
\alpha^{p} \beta^{q} \frac{d \overrightarrow{\mathfrak{w}}}{d \xi}=G(\alpha, \beta, \xi) \overrightarrow{\mathfrak{w}},
$$

where $p$ and $q$ are positive integers and $G(\alpha, \beta, \xi)$ is a matrix function with certain properties.

Therefore, it is sufficient to study the systems of Type A, C, E, F and G.

\section{§33. Domain $\left(\mathbf{D}_{8}\right)$.}

We introduce a new independent variable and a new parameter by

$$
\left\{\begin{array}{l}
x_{1}=\varepsilon^{-1} x \\
\varepsilon_{1}=\varepsilon \tau^{-1}
\end{array}\right.
$$

Then, it is easily seen that the domain $\left(D_{8}\right)$ becomes

$$
\left|\varepsilon_{1}\right| \leqq a, \quad 0<|\tau| \leqq b_{0}, \quad c \leqq\left|x_{1}\right| \leqq C
$$

and the system (WB) in $\S 2$ is reduced to 
$(\mathfrak{B})^{\prime}$

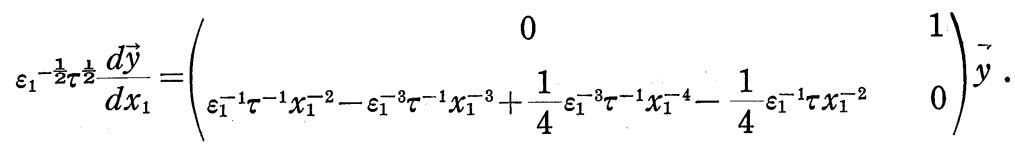

This equation has no singularity with respect to $x_{1}$ in the considered domain. Therefore, we construct a characteristic polygon of $(\mathfrak{W})^{\prime}$ with respect to $\varepsilon_{1}$ and $\tau$. As is shown in figure, the characteristic polygon has only one vertex:

$$
\mathrm{Q}_{0}=\left(-\frac{3}{2},-\frac{1}{2}\right) \text {. }
$$

Therefore, noticing that the coordinate

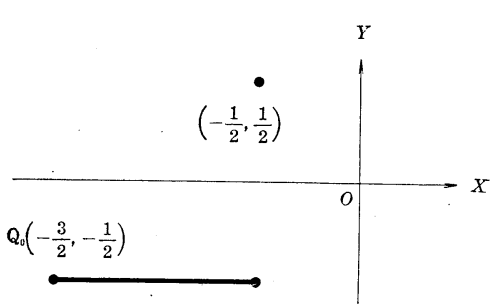

Fig. 13 of the vertex $Q_{0}$ is $\left(-\frac{3}{2},-\frac{1}{2}\right)$, we make a transformation

$$
\vec{y}=\left(\begin{array}{cc}
1 & 0 \\
0 & \varepsilon_{1}^{-\frac{3}{2} \tau} \tau^{-\frac{1}{2}}
\end{array}\right) \vec{z} .
$$

Then, we have the following:

$$
\varepsilon_{1} \tau \frac{d \vec{z}}{d x_{1}}=\left(\begin{array}{cc}
0 & 1 \\
\varepsilon_{1}^{2} x_{1}^{-2}-x_{1}^{-3}+\frac{1}{4} x_{1}^{-4}-\frac{1}{4} \varepsilon_{1}^{2} \tau^{2} x_{1}^{-2} & 0
\end{array}\right) \vec{z} .
$$

As $\varepsilon_{1}$ tends to 0 , the coefficient matrix will be reduced to

$$
\left(\begin{array}{cc}
0 & 1 \\
\frac{1}{4} x_{1}^{-4}\left(1-4 x_{1}\right) & 0
\end{array}\right) \text {. }
$$

Therefore, if $x_{1} \neq 1 / 4$, this matrix has two distinct characteristic values.

Keeping this fact in mind, we divide the domain $\left(\widetilde{\mathbf{D}}_{8}\right)$ into following two subdomains:

$$
\begin{aligned}
& \left|x_{1}-\frac{1}{4}\right| \geqq c_{1} ; \\
& \left|x_{1}-\frac{1}{4}\right| \leqq c_{1} .
\end{aligned}
$$

§ 34. Domain $\left(\mathbf{I}_{1}\right)$.

\section{$1^{\circ}$. Reduced form of Type G.}

We consider the system (33.3) in the domain $\left(I_{1}\right)$. Then, we see immediately that the coefficient matrix of the system (33.3) is a polynomial of $\varepsilon_{1}$ and $\tau$ whose coefficients also are polynomials of $x_{1}^{-1}$ and, moreover, has two distinct characteristic values in the domain $\left(I_{1}\right)$.

Therefore, in order to study the behavior of solutions of the system (33.3) in the domain $\left(I_{1}\right)$, it is sufficient to consider a system of Type $G$ :

$$
\alpha^{p} \beta^{q} \frac{d \overrightarrow{\mathfrak{w}}}{d \xi}=G(\alpha, \beta, \xi) \overrightarrow{\mathfrak{w}},
$$


where we suppose that

1) $p$ and $q$ are positive integers ;

2) $G(\alpha, \beta, \xi)$ is a matrix function which is holomorphic and bounded in $(\alpha, \beta, \xi)$ for

$$
\alpha \in \mathfrak{D}\left(\Theta_{ \pm} ; a\right), \quad \beta \in \mathscr{D}\left(\Theta_{ \pm}^{\prime} ; b\right), \quad \xi \in \mathcal{K}
$$

and, moreover, asymptotically developable in powers of $\alpha$ :

$$
G(\alpha, \beta, \xi) \sim \sum_{h=0}^{\infty} \alpha^{h} G_{h}(\beta, \xi),
$$

uniformly valid for (34.1), as a tends to 0 in (34.1); $(\beta, \xi)$ for

3) $G_{h}(\beta, \xi)$ are matrix functions which are holomorphic and bounded in

$$
\beta \in \mathfrak{D}\left(\Theta_{ \pm}^{\prime} ; b\right), \quad \xi \in \mathcal{K}
$$

and admit uniform asymptotic expansions in powers of $\beta$ :

$$
G_{h}(\beta, \xi) \sim \sum_{k=0}^{\infty} \beta^{k} G_{h l}(\xi)
$$

as $\beta$ tends to 0 in the domain (34.2), where $G_{h k}(\xi)$ are matrix functions holomorphic and bounded in $\xi$ for

$$
\xi \in \mathcal{K}
$$

4) $G_{00}(\xi)$ has two distinct characteristic values in (34.3);

5) $\mathcal{K}$ is a simply connected, bounded and open region.

Remark. In the present case, it is sufficient to take a following domain as the domain $\mathcal{K}$ :

$$
\left\{\xi: c^{\prime}<|\xi|<C^{\prime}, \Theta_{-}^{\prime \prime}<\arg \xi<\Theta_{+}^{\prime \prime}\right\},
$$

where $\left(c^{\prime}=c, C^{\prime}=C\right)$ or $\left(c^{\prime}=c, C^{\prime}=\frac{1}{4}-c_{1}\right.$ or $\left.c^{\prime}=\frac{1}{4}+c_{1}, C^{\prime}=C\right)$ according as we have $\arg \xi=0$ or not (cf. Fig. 8 in $\S 20$ ).

$\mathbf{2}^{\circ}$. Thus we have the following:

XVIII. In the subdomain

$$
\left\{\begin{array}{l}
\left|\varepsilon \tau^{-1}\right| \leqq a, \quad c \leqq\left|\varepsilon^{-1} x\right| \leqq C, \\
\left|\varepsilon^{-1} x-\frac{1}{4}\right| \geqq c_{1},
\end{array}\right.
$$

the system (W)' will be reduced to a system of Type $\mathrm{G}$ by a transformation:

$$
\left\{\begin{array}{l}
\vec{y}=\left(\begin{array}{cc}
1 & 0 \\
0 & \varepsilon_{1}^{-\frac{3}{2}} \tau^{-\frac{1}{2}}
\end{array}\right) \overrightarrow{\mathfrak{w}} \\
\varepsilon_{1}\left(=\varepsilon \tau^{-1}\right)=\xi, \\
\tau=\beta, \\
x_{1}\left(=\varepsilon^{-1} x\right)=\xi .
\end{array}\right.
$$

§35. Domain $\left(\mathbf{I}_{2}\right)$.

We consider again the system (33.3) in the domain $\left(I_{2}\right)$ and introduce a new 
independent variable :

$$
x_{2}=x_{1}-\frac{1}{4}
$$

Then, the considered domain becomes

$\left(\widetilde{\mathrm{I}_{2}}\right)$

$$
\left|\dot{\varepsilon_{1}}\right| \leqq a, \quad 0<|\tau| \leqq b_{0}, \quad\left|x_{2}\right| \leqq c_{1}
$$

and the system (33.3) is written as

$$
\varepsilon_{1} \tau \frac{d \vec{z}}{d x_{2}}=\left(\begin{array}{cc}
0 & 1 \\
\frac{4 \varepsilon_{1}^{2}\left(4-\tau^{2}\right)}{\left(1+4 x_{2}\right)^{2}}-\frac{256 x_{2}}{\left(1+4 x_{2}\right)^{4}} & 0
\end{array}\right) \vec{z} .
$$

\section{$1^{\circ}$. Characteristic polyhedron of (35.2).}

Let us construct a characteristic polyhedron of (35.2). However, since $x_{2}=0$ is a regular point of (35.2), it is sufficient to consider a modified characteristic polyhedron. Then, it is easily seen that the modified characteristic polyhedron has three vertices:

$$
\mathrm{V}_{1}=\left(0,0, \frac{1}{2}\right), \quad \mathrm{V}_{2}=(1,0,0), \quad \mathrm{V}_{\mathrm{3}}=\left(\frac{1}{3}, \frac{1}{3}, 0\right)
$$

as is shown in figure.

As is shown in the figure, at the vertex $V_{1}$ four sides meet. Let $\mathrm{S}_{1}, \mathrm{~S}_{2}, \mathrm{~S}_{3}, \mathrm{~S}_{4}$ be such four sides. Then, the equation of those sides will be written as

$$
\begin{array}{ll}
\mathrm{S}_{1}: \quad Y & =0 ; \\
\mathrm{S}_{2}: X+2 Y+2 Z & =1 ; \\
\mathrm{S}_{3}: 3 X & +2 Z=1 ; \\
\mathrm{S}_{4}: X & =0 .
\end{array}
$$

We consider two characteristic trigonal pyramids - the one formed by three planes $\mathrm{S}_{1}, \mathrm{~S}_{2}, \mathrm{~S}_{4}$ and the other formed by $\mathrm{S}_{2}, \mathrm{~S}_{3}, \mathrm{~S}_{4}$. Then, we see, from a simple calculation, that the directional parameters of the three edges of such two trigonal pyramids are given by

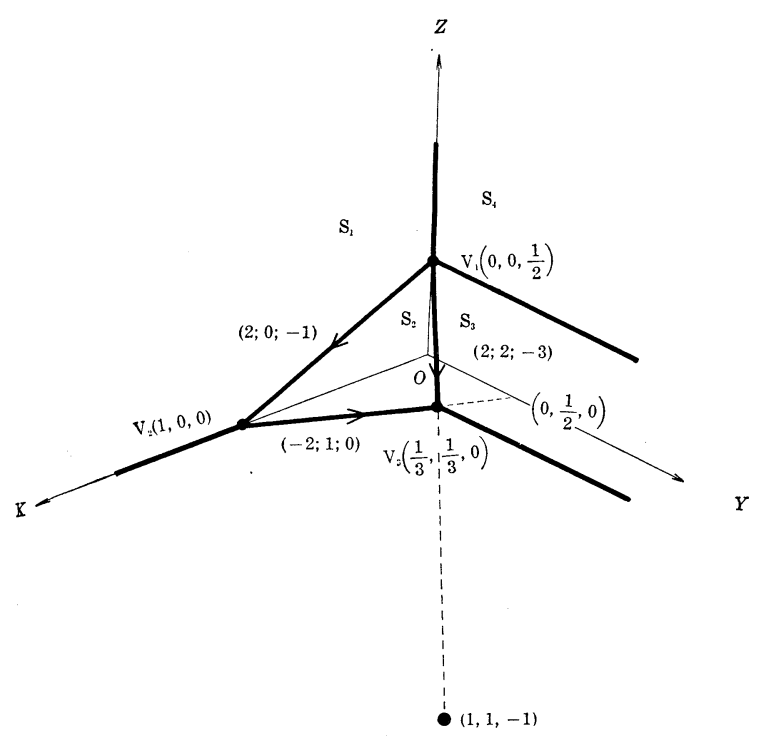

Fig. 14

$$
(2 ; 0 ;-1), \quad(0 ; 1 ;-1), \quad(0 ; 0 ; 1), \quad \mathrm{S}_{1} \cup \mathrm{S}_{2} \cup \mathrm{S}_{4}
$$

and

$$
(2 ; 2 ;-3), \quad(0 ; 1 ; 0), \quad(0 ;-1 ; 1), \quad \mathrm{S}_{2} \cup \mathrm{S}_{3} \cup \mathrm{S}_{4}
$$

respectively. On the other hand, at each of the vertices $V_{2}$ and $V_{3}$, there exists only one characteristic trigonal pyramid. As is easily seen from the 
figure, the directional parameters of the three edges are

$$
(1 ; 0 ; 0), \quad(-2 ; 1 ; 0), \quad(-2 ; 0 ; 1), \quad \mathrm{V}_{2}
$$

and

$$
(2 ;-1 ; 0), \quad(0 ; 1 ; 0), \quad(-2 ;-2 ; 3), \quad \mathrm{V}_{3}
$$

respectively.

$2^{\circ}$. Division of the domain $\left(\tilde{\mathbf{I}}_{2}\right)$.

We obtain following four principal subdomains :

$$
\begin{aligned}
& \left|\varepsilon_{1}^{2} x_{2}^{-1}\right| \leqq a_{3}, \quad\left|\tau x_{2}^{-1}\right| \leqq B_{3} ; \\
& \left|\varepsilon_{1}^{2} \tau^{2} x_{2}^{-3}\right| \leqq a_{4}, \quad\left|\tau^{-1} x_{2}\right| \leqq c_{4} ; \\
& \left|\varepsilon_{1}^{-2} \tau\right| \leqq B_{5} ; \quad\left|\varepsilon_{1}^{-2} x_{2}\right| \leqq c_{5} ;
\end{aligned}
$$

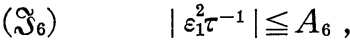

$$
\begin{aligned}
& \left|\varepsilon_{1}^{-2} \tau^{-2} x_{2}^{3}\right| \leqq C_{6},
\end{aligned}
$$

which correspond the above four characteristic trigonal pyramids respectively.

Further, we add a following auxiliary subdomain:

$$
\left|\varepsilon_{1}^{-2} \tau\right| \leqq B_{7}, \quad c_{7} \leqq\left|\varepsilon_{1}^{-2} x_{2}\right| \leqq C_{7} .
$$

$3^{\circ}$. Possibility of covering of the domain $\left(\tilde{\mathbf{I}}_{2}\right)$.

The union of these five subdomains covers the domain $\left(\widetilde{\mathbf{I}}_{2}\right)$ without lacuna.

Indeed, this assertion follows at once from the following:

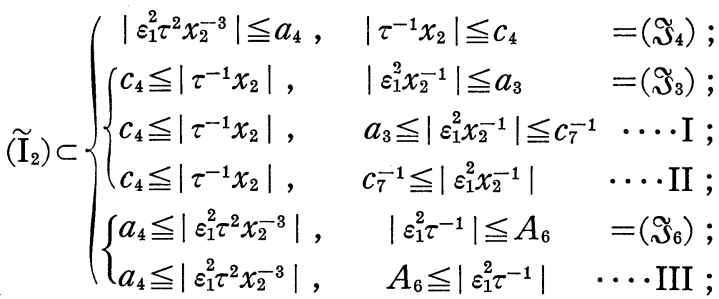

$$
\begin{aligned}
& \mathrm{I} \subset a_{3} c_{4} \leqq\left|\varepsilon_{1}^{2} \tau^{-1}\right|, \quad a_{3} \leqq\left|\varepsilon_{1}^{2} x_{2}^{-1}\right| \leqq c_{7}^{-1}=\left(\Im_{7}\right) \text {; } \\
& \text { II } \subset c_{4} c_{7}^{-1} \leqq\left|\varepsilon_{1}^{2} \tau^{-1}\right|, \quad c_{7}^{-1} \leqq\left|\varepsilon_{1}^{2} x_{2}^{-1}\right| \quad=\left(\Im_{5}\right) \text {; } \\
& \text { III } \subset A_{6} \leqq\left|\varepsilon_{1}^{2} \tau^{-1}\right|, \quad A_{6}^{\frac{2}{3}} a_{4}^{\frac{1}{3}} \leqq\left|\varepsilon_{1}^{2} x_{2}^{-1}\right| \quad=\left(\Im_{5}\right) \text {. }
\end{aligned}
$$

\section{§36. Domain $\left(\mathfrak{\$}_{3}\right)$.}

We consider the system (35.2) in the domain

$$
\left|\varepsilon_{1}^{2} x_{2}^{-1}\right| \leqq a_{3}, \quad\left|\tau x_{2}^{-1}\right| \leqq B_{3} .
$$

Noticing that the coordinate of the vertex $\mathrm{V}_{1}$ is $\left(0,0, \frac{1}{2}\right)$, if we put

$$
\vec{z}=\left(\begin{array}{cc}
1 & 0 \\
0 & x_{2}^{\frac{1}{2}}
\end{array}\right) \vec{u}
$$

it follows from (35.2) that

$$
\left(\varepsilon_{1} \tau x_{2}-\frac{3}{2}\right) \cdot x_{2} \frac{d \vec{u}}{d x_{2}}=\left(\begin{array}{cc}
0 & 1 \\
L\left(\varepsilon_{1}, \tau, x_{2}\right) & -\frac{1}{2}\left(\varepsilon_{1} \tau x_{2}{ }^{-\frac{3}{2}}\right)
\end{array}\right) \vec{u},
$$


where

$$
L=\frac{16 \varepsilon_{1}^{2} x_{2}^{-1}-4 \varepsilon_{1}^{2} \tau^{2} x_{2}^{-1}}{\left(1+4 x_{2}\right)^{2}}-\frac{256}{\left(1+4 x_{2}\right)^{4}} .
$$

Since, by an elementary calculation, we can write

$$
\left\{\begin{array}{l}
\varepsilon_{1} \tau x_{2}^{-\frac{3}{2}}=\left(\varepsilon_{1}^{2} x_{2}^{-1}\right)^{\frac{1}{2}} \cdot\left(\tau x_{2}^{-1}\right), \\
\varepsilon_{1}^{2} \tau_{2} x_{2}^{-1}=\left(\varepsilon_{1}^{2} x_{2}^{-1}\right) \cdot\left(\tau x_{2}^{-1}\right)^{2} \cdot x_{2}^{2},
\end{array}\right.
$$

we see that the coefficient matrix has two distinct characteristic values in the domain $\left(\Im_{3}\right)$ provided that, no matter how $B_{3}$ is large, the quantity $a_{3}$ is sufficiently small. Further, this matrix is a polynomial of $\left(\varepsilon_{1}^{2} x_{2}^{-1}\right)^{\frac{1}{2}}$ and $\left(\tau x_{2}^{-1}\right)$ whose coefficients are functions developable in uniformly convergent power series of $x_{2}$. Hence, the system (36.2) (where we substitute $\varepsilon_{1}, \tau$ and $x_{2}$ by $\alpha^{2}, \beta^{2}$ and $\xi^{2}$ respectively) is a system of Type $A$.

Thus we have the following

XIX. In the subdomain

$$
\left\{\begin{array}{l}
\left|\varepsilon \tau^{-1}\right| \leqq a, \quad\left|\varepsilon^{-1} x-\frac{1}{4}\right| \leqq c_{1}, \\
\left|\left(\varepsilon \tau^{-1}\right)^{2}\left(\varepsilon^{-1} x-\frac{1}{4}\right)^{-1}\right| \leqq a_{3}, \quad\left|\tau\left(\varepsilon^{-1} x-\frac{1}{4}\right)^{-1}\right| \leqq B_{3},
\end{array}\right.
$$

the system (W) will be reduced to a system of Type A by a transformation

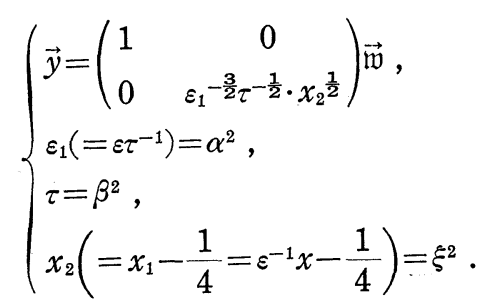

\section{§ 37. Domain $\left(\$_{4}\right)$.}

We consider again the system (36.2) in the domain

$$
\left|\varepsilon_{1}^{2} \tau^{2} x_{2}^{-3}\right| \leqq a_{4}, \quad\left|\tau^{-1} x_{2}\right| \leqq c_{4} .
$$

In this case we notice that we can write

$$
\left\{\begin{aligned}
\varepsilon_{1} \tau x_{2}-\frac{3}{2} & =\left(\varepsilon_{1}^{2} \tau^{2} x_{2}^{-3}\right)^{\frac{1}{2}} \cdot(\tau)^{0} \cdot\left(\tau^{-1} x_{2}\right)^{0}, \\
\varepsilon_{1}^{2} \tau^{2} x_{2}^{-1} & =\left(\varepsilon_{1}^{2} \tau^{2} x_{2}^{-3}\right) \tau^{2}\left(\tau^{-1} x_{2}\right)^{2}, \\
\varepsilon_{1}^{2} x_{2}^{-1} & =\left(\varepsilon_{1}^{2} \tau^{2} x_{2}^{-3}\right)\left(\tau^{-1} x_{2}\right)^{2} \\
x_{2} & =\tau\left(\tau^{-1} x_{2}\right) .
\end{aligned}\right.
$$

Hence, it is easily verified that the coefficient matrix of the system (36.2) has two distinct characteristic values in the domain $\left(\Im_{4}\right)$ and is a polynomial of $\left(\varepsilon_{1}^{2} \tau^{2} x_{2}^{-3}\right)^{\frac{1}{2}}$ whose coefficients are developable in uniformly convergent power series of $\left(\tau^{-1} x_{2}\right)$ (whose coefficients are polynomials of $\tau$ ). This assures us that the system (36.2) is a system of Type A if we replace $\varepsilon_{1}, \tau$ and $x_{2}$ by $\alpha^{2}, \beta^{2}$ and $\xi^{2}$ respectively. 
Thus we can state the following:

XX. In the subdomain

$\left(\Im_{4}\right)$

$$
\left\{\begin{array}{l}
\left|\varepsilon \tau^{-1}\right| \leqq a, \quad\left|\varepsilon^{-1} x-\frac{1}{4}\right| \leqq c_{1}, \\
\left|\varepsilon^{2}\left(\varepsilon^{-1} x-\frac{1}{4}\right)^{-3}\right| \leqq a_{4}, \quad\left|\tau^{-1}\left(\varepsilon^{-1} x-\frac{1}{4}\right)\right| \leqq c_{4},
\end{array}\right.
$$

the system (WW) will be reduced to a system of Type A by a transformation

$$
\left\{\begin{array}{l}
\vec{y}=\left(\begin{array}{cc}
1 & 0 \\
0 & \varepsilon_{1}^{-\frac{3}{2}} \tau^{-\frac{1}{2}} \cdot x_{2}^{\frac{1}{2}}
\end{array}\right) \overrightarrow{\mathfrak{w}}, \\
\varepsilon_{1}\left(=\varepsilon \tau^{-1}\right)=\alpha^{2} \\
\tau=\beta^{2} \\
x_{2}\left(=\varepsilon^{-1} x-\frac{1}{4}\right)=\xi^{2}
\end{array}\right.
$$

§ 38. Domain $\left(\$_{5}\right)$.

We consider again the system (35.2) in the domain

$$
\left|\varepsilon_{1}\right| \leqq a, \quad\left|\varepsilon_{1}^{-2} \tau\right| \leqq B_{5}, \quad\left|\varepsilon_{1}^{-2} x_{2}\right| \leqq c_{5} .
$$

Noticing that the coordinate of the vertex $V_{2}$ is $(1,0,0)$, let us make a transformation

$$
\vec{z}=\left(\begin{array}{ll}
1 & 0 \\
0 & \varepsilon_{1}
\end{array}\right) \vec{u}
$$

Then, the system (35.2) will be transformed into

$$
\tau \frac{d \vec{u}}{d x_{2}}=\left(\begin{array}{cc}
0 & 1 \\
\frac{4\left(4-\tau^{2}\right)}{\left(1+4 x_{2}\right)^{2}}-\frac{256 \varepsilon_{1}^{-2} x_{2}}{\left(1+4 x_{2}\right)^{4}} & 0
\end{array}\right) \vec{u} .
$$

Since we can write

$$
\left\{\begin{array}{c}
\tau=\varepsilon_{1}^{2}\left(\varepsilon_{1}^{-2} \tau\right), \\
x_{2}=\varepsilon_{1}^{2}\left(\varepsilon_{1}^{-2} x_{2}\right),
\end{array}\right.
$$

if we introduce a new parameter $\tau_{1}$ and a new independent variable $x_{3}$ by

$$
\left\{\begin{array}{l}
\tau_{1}=\varepsilon_{1}^{-2} \tau, \\
x_{3}=\varepsilon_{1}^{-2} x_{2}
\end{array}\right.
$$

the system (38.2) can be written as

$$
\tau_{1} \frac{d \vec{u}}{d x_{3}}=\left(\begin{array}{cc}
0 & 1 \\
\frac{16-4 \varepsilon_{1}^{4} \tau_{1}^{2}}{\left(1+4 \varepsilon_{1}^{2} x_{3}\right)^{2}}-\frac{256 x_{3}}{\left(1+4 \varepsilon_{1}^{2} x_{3}\right)^{4}} & 0
\end{array}\right) \vec{u}
$$

and the considered domain becomes

$$
\left|\varepsilon_{1}\right| \leqq a, \quad\left|\tau_{1}\right| \leqq B_{5}, \quad\left|x_{3}\right| \leqq c_{5} .
$$


From this we see immediately that the coefficient matrix of (38.3) is considered as a polynomial of $\tau_{1}$ whose coefficients are developable in uniformly convergent power series of $\varepsilon_{1}$ in the domain $\left(\widetilde{\Im}_{5}\right.$ ) (whose coefficients are polynomials of $x_{3}$ ). Further, this matrix has two distinct characteristic values in the domain $\left(\tilde{\widetilde{\Im}}_{5}\right)$ provided that, no matter how $B_{5}$ is large, $a$ and $c_{5}$ are sufficiently small. Hence, if we put

$$
\tau_{1}=\alpha, \quad \varepsilon_{1}=\beta, \quad x_{3}=\xi,
$$

the system (38.3) is of Type E.

Thus we can state the following:

XXI. In the subdomain

$$
\begin{cases}\left|\varepsilon \tau^{-1}\right| \leqq a, & \left|\varepsilon^{-1} x-\frac{1}{4}\right| \leqq c_{1}, \\ \left|\varepsilon^{-2} \tau^{3}\right| \leqq B_{5}, & \left|\left(\varepsilon \tau^{-1}\right)^{-2}\left(\varepsilon^{-1} x-\frac{1}{4}\right)\right| \leqq c_{5},\end{cases}
$$

the system (WB) will be reduced to a system of Type $\mathrm{E}$ by a change of variables:

$$
\left\{\begin{array}{l}
\vec{y}=\left(\begin{array}{lc}
1 & 0 \\
0 & \varepsilon_{1}-\frac{3}{2} \tau^{-\frac{1}{2}} \cdot \varepsilon_{1}
\end{array}\right) \overrightarrow{\mathfrak{w}}, \\
\varepsilon_{1}\left(=\varepsilon \tau^{-1}\right)=\beta, \\
\tau_{1}\left(=\varepsilon_{1}^{-2} \tau=\varepsilon^{-2} \tau^{3}\right)=\alpha, \\
x_{3}\left(=\varepsilon_{1}^{-2} x_{2}=\varepsilon_{1}^{-2}\left(x_{1}-\frac{1}{4}\right)=\left(\varepsilon \tau^{-1}\right)^{-2}\left(\varepsilon^{-1} x-\frac{1}{4}\right)\right)=\xi .
\end{array}\right.
$$

§ 39. Domain $\left(\Im_{6}\right)$.

We will study the system (35.2) in the domain

$$
\left|\varepsilon_{1}^{2} \tau^{-1}\right| \leqq A_{6}, \quad\left|\varepsilon_{1}^{-2} \tau^{-2} x_{2}^{3}\right| \leqq C_{6} .
$$

Noticing that the coordinate of the vertex $\mathrm{V}_{3}$ is $\left(\frac{1}{3}, \frac{1}{3}, 0\right)$, let us make a change of variables :

$$
\vec{z}=\left(\begin{array}{cc}
1 & 0 \\
0 & \varepsilon_{1}^{\frac{1}{3}} \tau^{\frac{1}{3}}
\end{array}\right) \vec{u} .
$$

Then, the system (35.2) will be transformed into

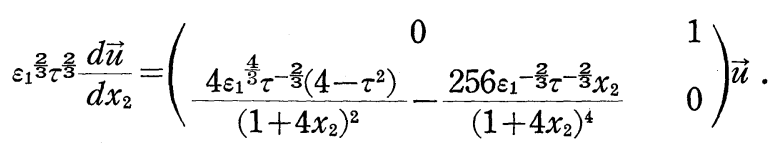

If we introduce a new parameter and a new independent variable by

$$
\left\{\begin{array}{l}
\varepsilon_{2}=\varepsilon_{1}^{2} \tau^{-1}, \\
x_{3}=\varepsilon_{1}^{-\frac{2}{3}} \tau^{-\frac{2}{3}} x_{2}
\end{array}\right.
$$

the domain $\left(\mathfrak{\Im}_{6}\right)$ is written as 


$$
\left|\varepsilon_{2}\right| \leqq A_{6}, \quad 0<|\tau| \leqq b_{0}, \quad\left|x_{3}\right| \leqq C_{6}^{\frac{1}{3}}
$$

and the system (39.2) is reduced to

$$
\frac{d \vec{u}}{d x_{3}}=\left(\begin{array}{cc}
0 & 1 \\
\frac{4 \varepsilon_{2}^{2}\left(4-\tau^{2}\right)}{\left(1+4 \varepsilon_{2}^{\frac{1}{3}} \tau x_{3}\right)^{2}}-\frac{256 x_{3}}{\left(1+4 \varepsilon_{2}^{\frac{3}{3}} \tau x_{3}\right)^{4}} & 0
\end{array}\right) \vec{u} .
$$

Hence, we see immediately that the system (39.4) (where we substitute $\varepsilon_{2}, \tau$ and $x_{3}$ by by $\alpha^{3}, \beta$ and $\xi$ respectively) is of Type $\mathrm{F}$.

Thus the following has been proved:

XXII. In the subdomain

$$
\left\{\begin{array}{l}
\left|\varepsilon \tau^{-1}\right| a, \quad\left|\varepsilon^{-1} x-\frac{1}{4}\right| \leqq c_{1}, \\
\left|\varepsilon^{2} \tau^{-3}\right| \leqq A_{6}, \quad\left|\varepsilon^{-2}\left(\varepsilon^{-1} x-\frac{1}{4}\right)^{3}\right| \leqq C_{6},
\end{array}\right.
$$

the system (WB) is reduced to a system of Type $\mathrm{F}$ if we put

$$
\left\{\begin{array}{l}
\vec{y}=\left(\begin{array}{ll}
1 & 0 \\
0 & \varepsilon_{1}-\frac{3}{2} \tau^{-\frac{1}{2}} \cdot \varepsilon_{1}^{\frac{1}{3}} \tau^{\frac{1}{3}}
\end{array}\right) \overrightarrow{\mathfrak{w}}, \\
\varepsilon_{2}\left(=\varepsilon_{1}^{2} \tau^{-1}=\left(\varepsilon \tau^{-1}\right)^{2} \tau^{-1}=\varepsilon^{2} \tau^{-3}\right)=\alpha^{3}, \\
\tau=\beta, \\
x_{3}\left(=\varepsilon_{1}-\frac{2}{3} \tau^{-\frac{2}{3}} x_{2}=\varepsilon_{1}-\frac{2}{3} \tau^{-\frac{2}{3}}\left(x_{1}-\frac{1}{4}\right)=\varepsilon^{-\frac{2}{3}}\left(\varepsilon^{-1} x-\frac{1}{4}\right)\right)=\xi .
\end{array}\right.
$$

§40. Domain $\left(\Im_{7}\right)$.

We consider the system (35.2) in the auxiliary subdomain $\left(\mathfrak{\Im}_{7}\right)$. This domain is defined by

$$
\left|\varepsilon_{1}^{-2} \tau\right| \leqq B_{7}, \quad c_{7} \leqq\left|\varepsilon_{1}^{-2} x_{2}\right| \leqq C_{7} .
$$

Noticing that the coordinate of the vertex $\mathrm{V}_{2}$ is $(1,0,0)$, we put

$$
\left\{\begin{array}{l}
\vec{z}=\left(\begin{array}{ll}
1 & 0 \\
0 & \varepsilon_{1}
\end{array}\right) \vec{u}, \\
\varepsilon_{1}^{-2} x_{2}=x_{4}, \\
\varepsilon_{1}^{-2} \tau=\tau_{2} .
\end{array}\right.
$$

Then, the transformed system can be written as

$$
\tau_{2} \frac{d \vec{u}}{d x_{4}}=\left(\begin{array}{cc}
0 & 1 \\
\frac{16\left\{\left(1+4 \varepsilon_{1}^{2} x_{4}\right)^{2}-16 x_{4}\right\}}{\left(1+4 \varepsilon_{1}^{2} x_{4}\right)^{4}}-\frac{4 \varepsilon_{1}^{4} \tau_{2}^{2}}{\left(1+4 \varepsilon_{1}^{2} x_{4}\right)^{2}} & 0
\end{array}\right) \vec{u}
$$

and the considered domain is reduced to

$$
\left|\varepsilon_{1}\right| \leqq a, \quad\left|\tau_{2}\right| \leqq B_{7}, \quad c_{7} \leqq\left|x_{4}\right| \leqq C_{7} .
$$

However, since the system (40.2) has no singularity with respect to $x_{4}$, we may take $c_{7}=0$. 
It is easily seen that, in the domain $\left(\widetilde{\widetilde{\Im}}_{7}\right)$, the system (40.2) may have a turning point in the neighborhood of a point $x_{4}=1 / 16$.

Indeed, such a turning point is given by the roots of the algebraic equation :

$$
16 \varepsilon_{1}^{4} x_{4}^{2}-8\left(2-\varepsilon_{1}^{2}\right) x_{4}+1=0 \text {. }
$$

These two roots are given by

$$
x_{4}=f_{ \pm}\left(\varepsilon_{1}\right),
$$

where

$$
f_{ \pm}\left(\varepsilon_{1}\right)=\frac{1}{4 \varepsilon^{4}}\left[2-\varepsilon_{1}^{2} \pm 2 \sqrt{1-\varepsilon_{1}^{2}}\right] .
$$

However, $f_{+}\left(\varepsilon_{1}\right)$ tends to infinity as $\varepsilon_{1}$ tends to 0 and, consequently, the point $x_{4}=f_{+}\left(\varepsilon_{1}\right)$ is not contained in the considered domain, while $f_{-}\left(\varepsilon_{1}\right)$ tends to $\frac{1}{16}$ as $\varepsilon_{1}$ tends to 0 and therefore we can suppose

$$
c_{7}<\left|f_{-}\left(\varepsilon_{1}\right)\right|<C_{7} .
$$

This shows that $x_{4}=f_{-}\left(\varepsilon_{1}\right)$ is only one turning point contained in the considered domain.

Now we divide the domain $\left(\widetilde{\Im}_{7}\right)$ into following two subdomains:

$$
\begin{aligned}
& \left|x_{4}-f_{-}\left(\varepsilon_{1}\right)\right| \geqq c_{2} ; \\
& \left|x_{4}-f_{-}\left(\varepsilon_{1}\right)\right| \leqq c_{2} .
\end{aligned}
$$

In the former domain, the coefficient matrix has two distinct characteristic values. Therefore, the system (40.2) is of Type $\mathrm{E}$ in the domain $\left(\mathrm{I}_{8}\right)$.

Thus we have proved the following:

XXIII. In the subdomain

$\left(\mathrm{I}_{8}\right)$

$$
\left\{\begin{array}{l}
\left|\varepsilon \tau^{-1}\right| \leqq a, \quad\left|\varepsilon^{-1} x-\frac{1}{4}\right| \leqq c_{1}, \\
\left|\varepsilon^{-2} \tau^{3}\right| \leqq B_{7}, \quad c_{7} \leqq\left|\left(\varepsilon \tau^{-1}\right)^{-2}\left(\varepsilon^{-1} x-\frac{1}{4}\right)\right| \leqq C_{7}, \\
\left|\left(\varepsilon \tau^{-1}\right)^{-2}\left(\varepsilon^{-1} x-\frac{1}{4}\right)-f_{-}\left(\varepsilon \tau^{-1}\right)\right| \geqq c_{2},
\end{array}\right.
$$

a transformation

$$
\left\{\begin{array}{l}
\vec{y}=\left(\begin{array}{cc}
1 & 0 \\
0 & \varepsilon_{1}^{-\frac{3}{2} \tau^{-\frac{1}{2}} \cdot \varepsilon_{1}}
\end{array}\right) \overrightarrow{\mathfrak{w}}, \\
\varepsilon_{1}\left(=\varepsilon \tau^{-1}\right)=\beta, \\
\tau_{2}\left(=\varepsilon_{1}^{-2} \tau=\left(\varepsilon \tau^{-1}\right)^{-2} \tau=\varepsilon^{-2} \tau^{3}\right)=\alpha, \\
x_{4}\left(=\varepsilon_{1}^{-2} x_{2}=\left(\varepsilon \tau^{-1}\right)^{-2}\left(\varepsilon^{-1} x-\frac{1}{4}\right)\right)=\xi
\end{array}\right.
$$

reduces the system (W) to a system of Type $\mathrm{E}$.

§41. Domain $\left(\mathbf{I}_{9}\right)$.

We will study the behavior of solutions of the system (40.2) in the domain 
$\left(\mathrm{I}_{9}\right)$

$$
\left|\varepsilon_{1}\right| \leqq a, \quad\left|\tau_{2}\right| \leqq B_{7}, \quad\left|x_{4}-f_{-}\left(\varepsilon_{1}\right)\right| \leqq c_{2} .
$$

To do this, we introduce a new independent variable:

$$
x_{5}=x_{4}-f_{-}\left(\varepsilon_{1}\right) \text {. }
$$

Then the system (40.2) will be reduced to

$$
\tau_{2} \frac{d \vec{u}}{d x_{5}}=\left(\begin{array}{cc}
0 & 1 \\
M\left(\varepsilon_{1}, \tau_{2}, x_{5}\right) & 0
\end{array}\right) \vec{u},
$$

where

$$
M=\frac{128\left[2 \varepsilon_{1}^{4} x_{5}^{2}+\left(4 \varepsilon_{1}^{4} f_{-}\left(\varepsilon_{1}\right)+\varepsilon_{1}^{2}-2\right) x_{5}\right]}{\left(1+4 \varepsilon_{1}^{2} f_{-}\left(\varepsilon_{1}\right)+4 \varepsilon_{1}^{2} x_{5}\right)^{4}}-\frac{4 \varepsilon_{1}^{4} \tau_{2}^{2}}{\left(1+4 \varepsilon_{1}^{2} f_{-}+4 \varepsilon_{1}^{2} x_{5}\right)^{2}}
$$

and the domain $\left(\mathrm{I}_{9}\right)$ becomes

$$
\left|\varepsilon_{1}\right| \leqq a, \quad\left|\tau_{2}\right| \leqq B_{7}, \quad\left|x_{5}\right| \leqq c_{2} .
$$

Let us construct a characteristic polyhedron. Since, in this case, the considered system has no singularity with respect to $x_{5}$, it is sufficient to consider a modified characteristic polyhedron. As is shown in figure, the modified characteristic polyhedron has two vertices :

$$
\mathrm{V}_{1}=\left(0,0, \frac{1}{2}\right), \quad \mathrm{V}_{2}=\left(0, \frac{1}{3}, 0\right)
$$

and is of the form of a trigonal cylinder with its base on $(Y, Z)$-plane.

Now we divide the domain $\left(\widetilde{\mathbf{I}}_{9}\right)$ into following two subdomains :

(ふ) $\left|\tau_{2} x_{5}-\frac{3}{2}\right| \leqq b_{10}$;

(ई11) $\quad\left|\tau_{2}^{-\frac{2}{3}} x_{5}\right| \leqq C_{11}\left(C_{11}=b_{10}^{-\frac{2}{3}}\right)$.

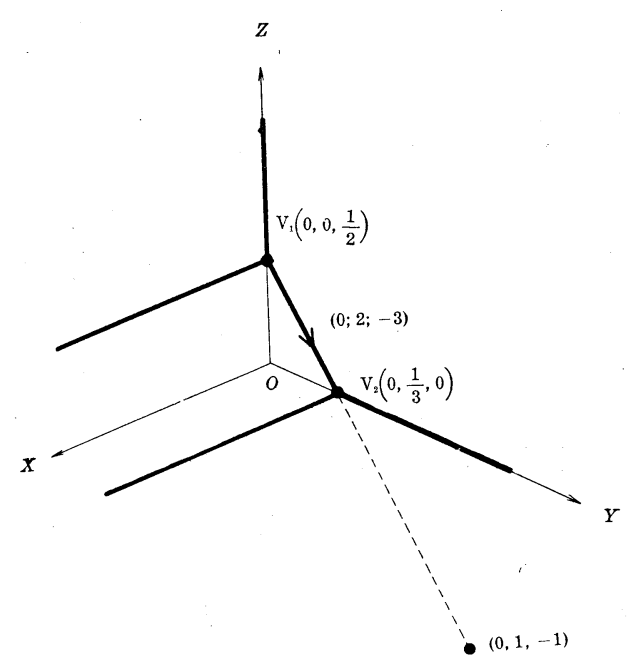

Fig. 15 .

Clearly, the union of these two subdomains covers the domain $\left(\widetilde{\Im}_{9}\right)$ without lacuna.

§ 42. Domain $\left(\Im_{10}\right)$.

Noticing that the coordinate of the vertex $V_{1}$ is $\left(0,0, \frac{1}{2}\right)$, we put

$$
\vec{u}=\left(\begin{array}{cc}
1 & 0 \\
0 & x_{5}^{\frac{1}{2}}
\end{array}\right) \vec{v} \text {. }
$$

Then, it follows from (41.2) that the transformed system will be written as

$$
\left(\tau_{2} x_{5}-\frac{3}{2}\right) \cdot x_{5} \frac{d \vec{v}}{d x_{5}}=\left(\begin{array}{cc}
0 & 1 \\
x_{5}{ }^{-1} M\left(\varepsilon_{1}, \tau_{2}, x_{5}\right) & -\frac{1}{2}\left(\tau_{2} x_{5}{ }^{-\frac{3}{2}}\right)
\end{array}\right) \vec{v}
$$

and 


$$
x_{5}^{-1} M=\frac{128\left(2 \varepsilon_{1}^{4} x_{5}+4 \varepsilon_{1}^{4} f_{-}\left(\varepsilon_{1}\right)+\varepsilon_{1}^{2}-2\right)}{\left(1+4 \varepsilon_{1}^{2} f_{-}\left(\varepsilon_{1}\right)+4 \varepsilon_{1}^{2} x_{5}\right)^{4}}-\frac{4 \varepsilon_{1}^{4} \tau_{2}^{2} x_{5}^{-1}}{\left(1+4 \varepsilon_{1}^{2} f_{-}+4 \varepsilon_{1}^{2} x_{5}\right)^{2}} .
$$

Since we can write

$$
\tau_{2}^{2} x_{5}^{-1}=\left(\tau_{2} x_{5}-\frac{3}{2}\right)^{2} \cdot x_{5}^{2},
$$

the coefficient matrix of (42.2) becomes a polynomial of $\left(\tau_{2} x_{5}{ }^{-\frac{3}{2}}\right)$ whose coefficients are developable in uniformly convergent power series of $x_{5}$ and $\varepsilon_{1}$ in the considered domain

$$
\left|\varepsilon_{1}\right| \leqq a, \quad\left|\tau_{2} x_{5}^{-\frac{3}{2}}\right| \leqq b_{10}, \quad\left|x_{5}\right| \leqq c_{2} .
$$

Further, this matrix has two distinct characteristic values in the domain $\left(\oiint_{10}\right)$. Hence, the system (42.2) is of Type C.

Thus, we have established the following:

XXIV. Consider the subdomain:

$\left(\Im_{10}\right)$

$$
\left\{\begin{array}{l}
\left|\varepsilon \tau^{-1}\right| \leqq a, \quad\left|\varepsilon^{-1} x-\frac{1}{4}\right| \leqq c_{1}, \\
\left|\varepsilon^{-2} \tau^{3}\right| \leqq B_{7}, \quad\left|\left(\varepsilon \tau^{-1}\right)^{-2}\left(\varepsilon^{-1} x-\frac{1}{4}\right)-f_{-}\left(\varepsilon \tau^{-1}\right)\right| \leqq c_{2}, \\
\left|\varepsilon^{-2} \tau^{3}\left\{\left(\varepsilon \tau^{-1}\right)^{-2}\left(\varepsilon^{-1} x-\frac{1}{4}\right)-f_{-}\left(\varepsilon \tau^{-1}\right)\right\}^{-\frac{3}{2}}\right| \leqq b_{10} .
\end{array}\right.
$$

Then, the system (W) is reduced to a system of Type $\mathrm{C}$ if we make a transformation

$$
\left\{\begin{array}{l}
\vec{y}=\left(\begin{array}{lc}
1 & 0 \\
0 & \varepsilon_{1}^{-\frac{3}{2}} \tau^{-\frac{1}{2}} \cdot \varepsilon_{1} \cdot x_{5}^{\frac{1}{2}}
\end{array}\right) \mathfrak{w}, \\
\varepsilon_{1}\left(=\varepsilon \tau^{-1}\right)=\beta, \\
\tau_{2}\left(=\varepsilon_{1}^{-2} \tau=\varepsilon^{-2} \tau^{3}\right)=\alpha, \\
x_{5}\left(=x_{4}-f_{-}\left(\varepsilon_{1}\right)=\varepsilon_{1}^{-2} x_{2}-f_{-}\left(\varepsilon_{1}\right)=\left(\varepsilon \tau^{-1}\right)^{-2}\left(\varepsilon^{-1} x-\frac{1}{4}\right)-f_{-}\left(\varepsilon \tau^{-1}\right)\right)=\xi .
\end{array}\right.
$$

§43. Domain $\left(\Im_{11}\right)$.

We consider again the system (41.2). Noticing that the coordinate of the vertex $\mathrm{V}_{2}$ is $\left(0, \frac{1}{3}, 0\right)$, if we put



the system (41.2) will be changed into

$$
\frac{d \vec{v}}{d x_{6}}=\left(\begin{array}{cc}
0 & 1 \\
\tau_{2}^{-\frac{2}{3}} M\left(\varepsilon_{1}, \tau_{2}, \tau_{2}^{\frac{2}{3}} x_{6}\right) & 0
\end{array}\right) \vec{v},
$$

where

$$
\tau_{2}^{-\frac{2}{3}} M=\frac{128\left\{2 \varepsilon_{1}^{4} \tau_{2}^{\frac{2}{3}} x_{6}^{2}+\left(4 \varepsilon_{1}^{4} f_{-}\left(\varepsilon_{1}\right)+\varepsilon_{1}^{2}-2\right) x_{6}\right\}}{\left(1+4 \varepsilon_{1}^{2} f_{-}\left(\varepsilon_{1}\right)+4 \varepsilon_{1}^{2} \tau_{2}^{\frac{2}{3}} x_{6}\right)^{4}}-\frac{4 \varepsilon_{1}^{4} \tau_{2}^{\frac{4}{3}}}{\left(1+4 \varepsilon_{1}^{2} f_{-}+4 \varepsilon_{1}^{2} \tau_{2}^{\frac{2}{3}} x_{6}\right)^{2}}
$$


and the domain $\left(\Im_{11}\right)$ will be written as

$$
\left|\varepsilon_{1}\right| \leqq a, \quad\left|\tau_{2}\right| \leqq B_{7}, \quad\left|x_{6}\right| \leqq C_{11} .
$$

From this we see immediately that the system (43.2) is a system of Type F.

Thus we have the following:

$\mathbf{X X V}$. In the subdomain

$\left(\Im_{11}\right)$

$$
\left\{\begin{array}{l}
\left|\varepsilon \tau^{-1}\right| \leqq a, \quad\left|\varepsilon^{-1} x-\frac{1}{4}\right| \leqq c_{1}, \\
\left|\varepsilon^{-2} \tau^{3}\right| \leqq B_{7}, \quad\left|\left(\varepsilon \tau^{-1}\right)^{-2}\left(\varepsilon^{-1} x-\frac{1}{4}\right)-f_{-}\left(\varepsilon \tau^{-1}\right)\right| \leqq c_{2}, \\
\left|\left(\varepsilon^{-2} \tau^{3}\right)^{-\frac{2}{3}}\left\{\left(\varepsilon \tau^{-1}\right)^{-2}\left(\varepsilon^{-1} x-\frac{1}{4}\right)-f_{-}\left(\varepsilon \tau^{-1}\right)\right\}\right| \leqq C_{11},
\end{array}\right.
$$

the system (W) is transformed into a system of Type $\mathrm{F}$ if we make a change of variables :

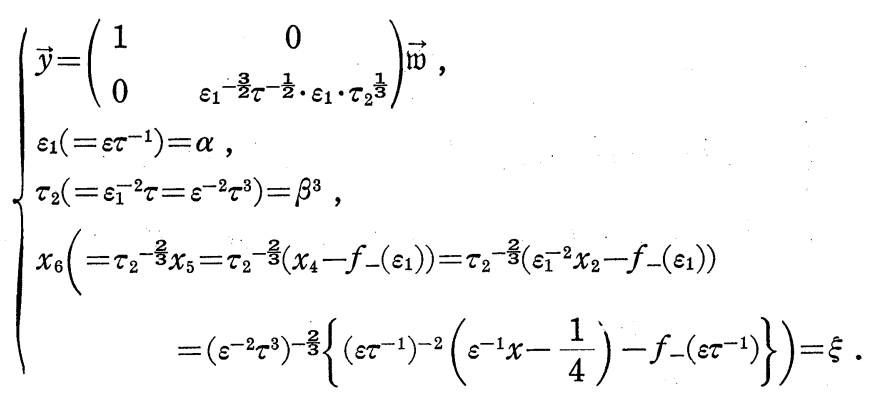

\section{§44. Conclusion.}

Summarizing the discussions done in this chapter, we can state the following

Theorem 2. In order to investigate the behavior of solutions of the Whittaker's differential equation in an auxiliary subdomain of the form

$$
\left|\varepsilon \tau^{-1}\right| \leqq a_{8}, \quad c_{8} \leqq\left|\varepsilon^{-1} x\right| \leqq C_{8},
$$

it is sufficient divide the domain $\left(\mathrm{D}_{8}\right)$ into eight subdomains $\mathrm{I}_{1}, \mathfrak{\Im}_{3}, \mathfrak{\Im}_{4}, \mathfrak{\Im}_{5}, \mathfrak{\Im}_{6}, \mathfrak{\Im}_{8}$, $\Im_{10}$ and $\Im_{11}$, and we have

$$
\begin{aligned}
\mathrm{D}_{8} & =\mathrm{I}_{1} \cup \mathrm{I}_{2}, \\
\mathrm{I}_{2} & \subset \Im_{3} \cup \mathfrak{\Im}_{4} \cup \mathfrak{\Im}_{5} \cup \Im_{6} \cup \Im_{7}, \\
\Im_{7} & =\mathrm{I}_{8} \cup \mathrm{I}_{9}, \\
\mathrm{I}_{9} & =\Im_{1_{0}} \cup \mathfrak{\Im}_{11} .
\end{aligned}
$$

The types of reduced forms in each of those subdomains are given by a following table.

\begin{tabular}{l|c|c|c|c|c|c|c|c}
\hline Subdomain & $\mathrm{I}_{1}$ & $\Im_{3}$ & $\Im_{4}$ & $\Im_{5}$ & $\Im_{6}$ & $\mathrm{I}_{8}$ & $\Im_{10}$ & $\Im_{11}$ \\
\hline Normal form & $\mathrm{G}$ & $\mathrm{A}$ & $\mathrm{A}$ & $\mathrm{E}$ & $\mathrm{F}$ & $\mathrm{E}$ & $\mathrm{C}$ & $\mathrm{F}$ \\
\hline Proposition & $\mathrm{XVIII}$ & $\mathrm{XIX}$ & $\mathrm{XX}$ & $\mathrm{XXI}$ & $\mathrm{XXII}$ & $\mathrm{XXIII}$ & $\mathrm{XXIV}$ & $\mathrm{XXV}$ \\
\hline Section & 34 & 36 & 37 & 38 & 39 & 40 & 42 & 43 \\
\hline
\end{tabular}




\section{Chapter VI. Study of the behavior of the solutions in the auxiliary subdomain $\left(D_{9}\right)$.}

In this chapter, we will show that the reduced froms of the Whittaker's differential equation in the domain $\left(D_{9}\right)$ are of Type $C, F, G$.

\section{§ 45. Domain $\left(\mathbf{D}_{9}\right)$.}

As we have already seen in $\S 10$, the auxiliary subdomain $\left(D_{9}\right)$ is defined by $\left(D_{9}\right)$

$$
c \leqq\left|\varepsilon^{\frac{1}{2}} \tau^{-1} x\right| \leqq C, \quad 0<|\varepsilon| \leqq a_{0}, \quad 0<|\tau| \leqq b_{0}, \quad 0<|x| \leqq c_{0} .
$$

We introduce a new independent variable $x_{1}$ :

$$
x_{1}=\varepsilon^{\frac{1}{2}} \tau^{-1} x \text {. }
$$

Then, the system (WB) in $\S 2$ will be reduced to a system

$$
\varepsilon \frac{d y}{d x_{1}}=\left(\begin{array}{cc}
0 & 1 \\
\varepsilon^{2} \tau^{-2} x_{1}^{-2}-\varepsilon^{\frac{3}{2}} \tau^{-1} x_{1}^{-3}+\frac{1}{4} \varepsilon^{3} \tau^{-2} x_{1}^{-4}-\frac{1}{4} \varepsilon^{2} x_{1}^{-2} & 0
\end{array}\right) \vec{y}
$$

and the domain $\left(\mathrm{D}_{9}\right)$ becomes

$$
0<|\varepsilon| \leqq a_{0}, \quad 0<|\tau| \leqq b_{0}, c \leqq\left|x_{1}\right| \leqq C .
$$

Since the system (WB) $)^{\prime}$ has no singularity with respect to $x_{1}$ in the considered domain, we construct a characteristic polygon with respect to $\varepsilon$ and $\tau$. Then, as is shown in figure, the characteristic polygon has two vertices:

$$
\mathrm{Q}_{0}=\left(\frac{3}{4},-\frac{1}{2}\right), \quad \mathrm{Q}_{1}=(1,-1) .
$$

We divide the domain $\left(\widetilde{\mathrm{D}}_{9}\right)$ into following two subdomains :

$$
\begin{gathered}
\left|\varepsilon^{\frac{1}{2}} \tau^{-1}\right| \leqq A_{1} \\
\left|\varepsilon^{-\frac{1}{2}} \tau\right| \leqq b_{2} .
\end{gathered}
$$

If we take $A_{1}=1 / b_{2}$, then the union of these two subdomains covers the domain $\left(\widetilde{\mathrm{D}}_{9}\right)$ without lacuna.



Fig 16

\section{§46. Domain $\left(\mathbf{J}_{2}\right)$.}

We consider firstly the subdomain $\left(\mathrm{J}_{2}\right)$. Observing that the subdomain $\left(\mathrm{J}_{2}\right)$ corresponds to the vertex $Q_{1}$, we make a transformation

$$
\left\{\begin{array}{c}
\vec{y}=\left(\begin{array}{cc}
1 & 0 \\
0 & \varepsilon \tau^{-1}
\end{array}\right) \vec{z} \\
\varepsilon^{-\frac{1}{2} \tau}=\tau_{1}
\end{array}\right.
$$

Then, we see that the system $(\mathfrak{W})^{\prime}$ is changed into 


$$
\varepsilon^{\frac{1}{2} \tau_{1} \frac{d \vec{z}}{d x_{1}}}=\left(\begin{array}{cc}
0 & 1 \\
x_{1}^{-3}-\tau_{1} x_{1}^{-3}+\frac{1}{4} \varepsilon x_{1}^{-4}-\frac{1}{4} \varepsilon \tau_{1}^{2} x_{1}^{-2} & 0
\end{array}\right) \vec{z}
$$

and the domain $\left(\mathrm{J}_{2}\right)$ is reduced to

$$
0<|\varepsilon| \leqq a_{0}, \quad\left|\tau_{1}\right| \leqq b_{2}, \quad c \leqq\left|x_{1}\right| \leqq C .
$$

Therefore, the coefficient matrix is a polynomial of $\varepsilon$ and $\tau_{1}$ whose coefficients also are polynomials of $x_{1}^{-1}$. Further, since $a_{0}$ and $b_{2}$ are supposed to be sufficiently small, the coefficient matrix has two distinct characteristic values in the domain $\left(\widetilde{J}_{2}\right)$. From this follows that the system (46.2) (where we put $\varepsilon=\alpha^{2}, \tau_{1}=\beta, x=\xi$ ) is a system of Type $\mathrm{G}$ in the considered domain.

Thus we have the following:

XXVI. In the subdomain

$$
\left|\varepsilon^{-\frac{1}{2}} \tau\right| \leqq b_{2}, \quad c \leqq\left|\varepsilon^{\frac{1}{2}} \tau^{-1} x\right| \leqq C,
$$

the system (M)' is reduced to a system of Type $\mathrm{G}$ if we put

$$
\left\{\begin{array}{l}
\vec{y}=\left(\begin{array}{cc}
1 & 0 \\
0 & \varepsilon \tau^{-1}
\end{array}\right) \overrightarrow{\mathfrak{w}}, \\
\varepsilon=\alpha^{2}, \\
\tau_{1}\left(=\varepsilon^{-\frac{1}{2}} \tau\right)=\beta, \\
x_{1}\left(=\varepsilon^{\frac{1}{2}} \tau^{-1} x\right)=\xi .
\end{array}\right.
$$

§ 47. Domain $\left(\mathbf{J}_{1}\right)$.

We consider the domain $\left(\mathrm{J}_{1}\right)$. Noticing that the coordinate of the vertex $\mathrm{Q}_{0}$ is $\left(\frac{3}{4},-\frac{1}{2}\right)$, let us make a transformation

$$
\left\{\begin{array}{l}
\vec{y}=\left(\begin{array}{cc}
1 & 0 \\
0 & \varepsilon^{\frac{3}{4}} \tau^{-\frac{1}{2}}
\end{array}\right) \vec{z} \\
\varepsilon^{\frac{1}{2} \tau^{-1}}=\varepsilon_{1}
\end{array}\right.
$$

Then, the system (WB) ${ }^{\prime}$ will be transformed into a system

$$
\varepsilon_{1}^{\frac{1}{2}} \tau \frac{d \vec{z}}{d x_{1}^{\prime}}=\left(\begin{array}{cc}
0 & 1 \\
\varepsilon_{1} x_{1}^{-2}-x_{1}^{-3}+\frac{1}{4} \varepsilon_{1}^{3} \tau^{2} x_{1}^{-4}-\frac{1}{4} \varepsilon_{1} \tau^{2} x_{1}^{-2} & 0
\end{array}\right) \vec{z}
$$

and the considered domain is reduced to

$$
\left|\varepsilon_{1}\right| \leqq A_{1}, \quad 0<|\tau| \leqq b_{0}, \quad c \leqq\left|x_{1}\right| \leqq C .
$$

As $\tau$ tends to 0 , the coefficient matrix will be reduced to

$$
\left(\begin{array}{cc}
0 & 1 \\
x_{1}^{-3}\left(\varepsilon_{1} x_{1}-1\right) & 0
\end{array}\right)
$$

Therefore, we see immediately that if $\varepsilon_{1} x_{1} \neq 1$, the coefficient matrix has two distinct characteristic values in the considered domain. Hence, in such a domain 
the system (47.2) becomes a system of Type G (if we substitute $\varepsilon_{1}$ by $\alpha^{2}$ ).

Keeping this fact in mind, we divide the domain $\left(\tilde{\mathrm{J}_{1}}\right)$ into following two subdomains :

$$
\begin{aligned}
& \left|\varepsilon_{1} x_{1}-1\right| \geqq c_{1} ; \\
& \left|\varepsilon_{1} x_{1}-1\right| \leqq c_{1} .
\end{aligned}
$$

Then we have immediately the following

XXVII. In the subdomain

$$
\left\{\begin{array}{l}
\left|\varepsilon^{\frac{1}{2}} \tau^{-1}\right| \leqq A_{1}, \quad c \leqq\left|\varepsilon^{\frac{1}{2}} \tau^{-1} x\right| \leqq C, \\
\left|\varepsilon \tau^{-2} x-1\right| \geqq c_{1}
\end{array}\right.
$$

the system (W)' is reduced to a system of Type $\mathrm{G}$ by a transformation

$$
\left\{\begin{array}{l}
\vec{y}=\left(\begin{array}{cc}
1 & 0 \\
0 & \varepsilon^{\frac{3}{4}} \tau^{-\frac{1}{2}}
\end{array}\right) \overrightarrow{\mathfrak{w}}, \\
\varepsilon_{1}\left(=\varepsilon^{\frac{1}{2}} \tau^{-1}\right)=\alpha^{2}, \\
\tau=\beta, \\
x_{1}\left(=\varepsilon^{\frac{1}{2}} \tau^{-1} x\right)=\xi .
\end{array}\right.
$$

\section{§48. Domain $\left(\mathbf{J}_{4}\right)$.}

We study the system (47.2) in the domain

$$
\left\{\begin{array}{l}
\left|\varepsilon_{1}\right| \leqq A_{1}, \quad 0<|\tau| \leqq b_{0}, \quad c \leqq\left|x_{1}\right| \leqq C, \\
\left|\varepsilon_{1} x_{1}-1\right| \leqq c_{1}
\end{array}\right.
$$

To do this, introduce a new independent variable :

$$
x_{2}=\varepsilon_{1} x_{1}-1 \text {. }
$$

Then, the system (47.2) is written as

$$
\varepsilon_{1}^{\frac{3}{2} \tau} \frac{d \vec{z}}{d x_{2}}=\left(\begin{array}{cc}
0 & 1 \\
\frac{\varepsilon_{1}^{7} \tau^{2}}{4\left(1+x_{2}\right)^{4}}+\frac{\varepsilon_{1}^{3} x_{2}}{\left(1+x_{2}\right)^{3}}-\frac{\varepsilon_{1}^{3} \tau^{2}}{4\left(1+x_{2}\right)^{2}} & 0
\end{array}\right) \vec{z}
$$

and the domain $\left(\mathrm{J}_{4}\right)$ is reduced to

$$
\left|\varepsilon_{1}\right| \leqq A_{1}, \quad 0<|\tau| \leqq b_{0}, \quad\left|x_{2}\right| \leqq c_{1} .
$$

Further we put

$$
\vec{z}=\left(\begin{array}{cc}
1 & 0 \\
0 & \varepsilon_{1^{\frac{3}{2}}}^{\frac{3}{2}}
\end{array}\right) \vec{u} .
$$

The system satisfied by $\vec{u}$ is easily found to be

$$
\tau \frac{d \vec{u}}{d x_{2}}=\left(\begin{array}{cc}
0 & 1 \\
\frac{\varepsilon_{1}^{4} \tau^{2}}{4\left(1+x_{2}\right)^{4}}+\frac{x_{2}}{\left(1+x_{2}\right)^{3}}-\frac{\tau^{2}}{4\left(1+x_{2}\right)^{2}} & 0
\end{array}\right) \vec{u}
$$

$1^{\circ}$. Characteristic polyhedron of the system (48.4).

Let us construct a characteristic polyhedron. However, in the present case, 
it is sufficient to construct a modified characteristic polyhedron. As is shown in figure, the modified characteristic polyhedron has two vertices:

$$
\mathrm{V}_{1}=\left(0,0, \frac{1}{2}\right), \quad \mathrm{V}_{2}=\left(0, \frac{1}{3}, 0\right)
$$

and is regarded as a trigonal cylinder with its base on $(Y, Z)$-plane.

$2^{\circ}$. Division of the domain $\left(\widetilde{\mathbf{J}}_{4}\right)$.

As is shown in the figure, at each of the vertices $V_{1}$ and $V_{2}$ there exists only one characteristic trigonal pyramid. The directional parameters of the three edges of such pyramids are given by

$$
\begin{array}{lll}
(1 ; 0 ; 0), & (0 ; 2 ;-3), \quad(0 ; 0 ; 1) \\
(1 ; 0 ; 0), & (0 ; 1 ; 0), \quad(0 ;-2 ; 3) .
\end{array}
$$

Therefore the corresponding principal subdomains are written as

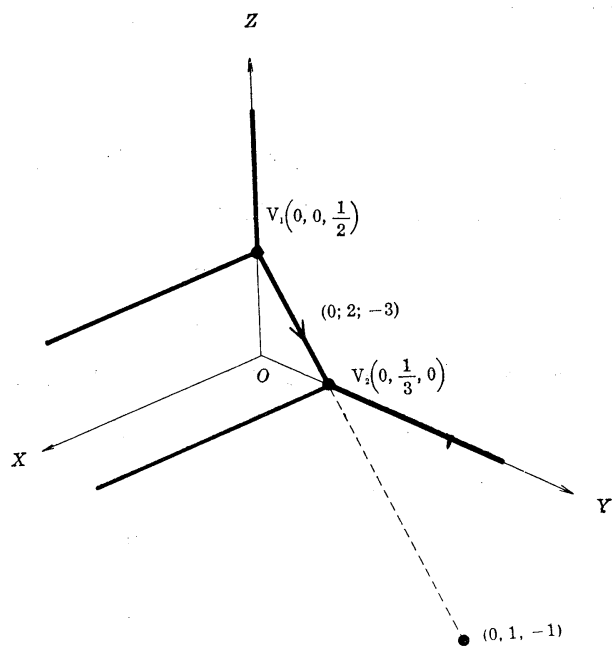

(ふ) $\quad\left|\tau x_{2}^{-\frac{3}{2}}\right| \leqq b_{5}$;

$$
\left|\tau^{-\frac{2}{3}} x_{2}\right| \leqq C_{6} \quad\left(C_{6}=b_{5}^{-\frac{2}{3}}\right) .
$$

Fig. 17

Obviously, the domain $\left(\widetilde{\Im}_{4}\right)$ is covered by these two subdomains without lacuna.

$\S 49$. Domain $\left(\Im_{5}\right)$.

Noticing that the coordinate of the vertex $V_{1}$ is $\left(0,0, \frac{1}{2}\right)$, let us make a transformation

$$
\vec{u}=\left(\begin{array}{ll}
1 & 0 \\
0 & x_{2}^{\frac{1}{2}}
\end{array}\right) \vec{v}
$$

Then, the system (48.4) is reduced to

$$
\left(\tau x_{2}^{-\frac{3}{2}}\right) \cdot x_{2} \frac{d \vec{v}}{d x_{2}}=\left(\begin{array}{cc}
0 & 1 \\
\frac{\varepsilon_{1}^{4} \tau^{2} x_{2}^{-1}}{4\left(1+x_{2}\right)^{4}}+\frac{1}{\left(1+x_{2}\right)^{3}}-\frac{\tau^{2} x_{2}^{-1}}{4\left(1+x_{2}\right)^{2}} & -\frac{1}{2}\left(\tau x_{2}^{-\frac{3}{2}}\right)
\end{array}\right) \vec{v} .
$$

Since we can write

$$
\tau^{2} x_{2}^{-1}=\left(\tau x_{2}^{-\frac{3}{2}}\right)^{2} x_{2}^{2},
$$

we see immediately that the coefficient matrix is a polynomial of $\left(\tau x_{2}^{-\frac{3}{2}}\right)$ and $\varepsilon_{1}$ whose coefficients are developable in a uniformly convergent power series of $x_{2}$. Moreover, as $\left(\tau x_{2}^{-\frac{3}{2}}\right), \varepsilon_{1}$ and $x_{2}$ tend to 0 , the coefficient matrix tends to

$$
\left(\begin{array}{ll}
0 & 1 \\
1 & 0
\end{array}\right)
$$

which has two distinct characteristic values \pm 1 . Therefore the system (49.2) is of Type $\mathrm{C}$ (where we have to put $\nu=0, q=0$ ). 
Thus we can state the following:

XXVIII. In the subdomain

$\left(\Im_{5}\right)$

$$
\left\{\begin{array}{l}
\left|\varepsilon^{\frac{1}{2}} \tau^{-1}\right| \leqq A_{1}, \quad\left|\varepsilon \tau^{-2} x-1\right| \leqq c_{1}, \\
\left|\tau\left(\varepsilon \tau^{-2} x-1\right)^{-\frac{3}{2}}\right| \leqq b_{5}
\end{array}\right.
$$

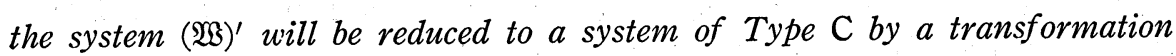

$$
\left\{\begin{array}{l}
\vec{y}=\left(\begin{array}{cc}
1 & 0 \\
0 & \varepsilon^{\frac{3}{4}} \tau^{-\frac{1}{2}} \cdot \varepsilon_{1}^{\frac{3}{2}} \cdot x_{2}
\end{array}\right) \overrightarrow{\mathfrak{w}}, \\
\varepsilon_{1}\left(=\varepsilon^{\frac{1}{2}} \tau^{-1}\right)=\beta, \\
\tau=\alpha, \\
x_{2}\left(=\varepsilon_{1} x_{1}-1=\varepsilon_{1} \cdot \varepsilon^{\frac{1}{2}} \tau^{-1} x-1=\varepsilon \tau^{-2} x-1\right)=\xi .
\end{array}\right.
$$

§50. Domain (\$ั $)_{6}$.

Noticing that the coordinate of the vertex $\mathrm{V}_{2}$ is $\left(0, \frac{1}{3}, 0\right)$, let us put

$$
\vec{u}=\left(\begin{array}{cc}
1 & 0 \\
0 & \tau^{\frac{1}{3}}
\end{array}\right) \vec{v}
$$

Then, the system (48.4) is changed into a system

$$
\tau^{\frac{2}{3}} \frac{d \vec{v}}{d x_{2}}=\left(\begin{array}{cc}
0 & 1 \\
\frac{\varepsilon_{1}^{4} \tau^{\frac{4}{3}}}{4\left(1+x_{2}\right)^{4}}+\frac{\tau^{-\frac{2}{3}} x_{2}}{\left(1+x_{2}\right)^{3}}-\frac{\tau^{\frac{4}{3}}}{4\left(1+x_{2}\right)^{2}} & 0
\end{array}\right) \vec{v} .
$$

If we introduce a new independent variable

$$
x_{3}=\tau^{-\frac{2}{3}} x_{2}
$$

the system (50.2) will be written as

$$
\frac{d v}{d x_{3}}=\left(\begin{array}{cc}
0 & 1 \\
\frac{\varepsilon_{1}^{4} \tau^{\frac{4}{3}}}{4\left(1+\tau^{\frac{2}{3}} x_{3}\right)^{4}}+\frac{x_{3}}{\left(1+\tau^{\frac{2}{3}} x_{3}\right)^{3}}-\frac{\tau^{\frac{4}{3}}}{4\left(1+\tau^{\frac{2}{3}} x_{3}\right)^{2}} & 0
\end{array}\right) \vec{v}
$$

and the considered domain becomes

$$
\left(\widetilde{\Im}_{6}\right) \quad\left|\varepsilon_{1}\right| \leqq A_{1}, \quad|\tau| \leqq b_{0}, \quad\left|x_{3}\right| \leqq C_{6} \text {. }
$$

Therefore, this is of Type $\mathrm{F}$.

Thus we have the following

XXIX. In the subdomain

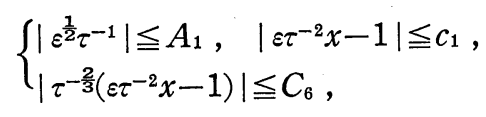

a transformation

$$
\left\{\begin{array}{l}
\vec{y}=\left(\begin{array}{lc}
1 & 0 \\
0 & \varepsilon^{\frac{3}{4}} \tau^{-\frac{1}{2}} \cdot \varepsilon_{1}^{\frac{3}{2}} \cdot \tau^{\frac{1}{3}}
\end{array}\right) \overrightarrow{\mathfrak{w}}, \\
\varepsilon_{1}\left(=\varepsilon^{\frac{1}{2} \tau^{-1}}\right)=\alpha, \\
\tau=\beta^{3}, \\
x_{3}\left(=\tau^{-\frac{2}{3}} x_{2}=\tau^{-\frac{2}{3}}\left(\varepsilon_{1} x_{1}-1\right)=\tau^{-\frac{2}{3}}\left(\varepsilon \tau^{-2} x-1\right)\right)=\xi
\end{array}\right.
$$


changes the system (W)' into a system of Type $\mathrm{F}$.

\section{$\S 51$. Conclusion.}

Summarizing the results obtained in this chapter, we can state the following :

Theorem 3. In order to investigate the behavior of solutions of the Whittaker's differential equation (W) in the auxiliary subdomain

$$
c_{9} \leqq\left|\varepsilon \tau^{-2} x^{2}\right| \leqq C_{9}, \quad 0<|\varepsilon| \leqq a_{0}, \quad 0<|\tau| \leqq b_{0}, \quad 0<|x| \leqq c_{0},
$$

we divide the domain $\left(\mathrm{D}_{9}\right)$ into four subdomains. Such subdomains and the types of the reduced form in each of these subdomains are given by a following table:

\begin{tabular}{l|c|c|c|c}
\hline Subdomain & $\mathrm{J}_{2}$ & $\mathrm{~J}_{3}$ & $\Im_{5}$ & $\Im_{6}$ \\
\hline Normal form & $\mathrm{G}$ & $\mathrm{G}$ & $\mathrm{C}$ & $\mathrm{F}$ \\
\hline Proposition & $\mathrm{XXVI}$ & $\mathrm{XXVII}$ & XXVIII & XXIX \\
\hline Section & 46 & 47 & 49 & 50 \\
\hline
\end{tabular}

Further, these subdomains are related in a following way:

$$
\begin{aligned}
\mathrm{D}_{9} & =\mathrm{J}_{1} \cup \mathrm{J}_{2}, \\
\mathrm{~J}_{1} & =\mathrm{J}_{3} \cup \mathrm{J}_{4}, \\
\mathrm{~J}_{4} & =\Im_{5} \cup \Im_{6} .
\end{aligned}
$$

\section{Chapter VII. Study of the behavior of the solutions} in the auxiliary subdomain $\left(D_{10}\right)$.

In this chapter we will show that, in order to construct an asymptotic expression of a fundamental set of independent solutions in $\left(D_{10}\right)$, it is sufficient to consider the same problem for the systems of Type A, C, E, F and G.

§52. Domain $\left(\mathbf{D}_{10}\right)$.

The auxiliary subdomain $\left(D_{10}\right)$ can be written as

$$
c \leqq\left|\tau^{-1} x\right| \leqq C, \quad 0<|\varepsilon| \leqq a_{0}, \quad 0<|\tau| \leqq b_{0}, \quad 0<|x| \leqq c_{0} .
$$

If we introduce a new independent variable:

$$
x_{1}=\tau^{-1} x,
$$

the domain $\left(\mathrm{D}_{10}\right)$ is reduced to

$$
0<|\varepsilon| \leqq a_{0}, \quad 0<|\tau| \leqq b_{0}, \quad c \leqq\left|x_{1}\right| \leqq C
$$

and the system (W) becomes

$$
\varepsilon^{\frac{1}{2}} \frac{d \vec{y}}{d x_{1}}=\left(\begin{array}{c:c}
0 & 1 \\
\varepsilon \tau^{-2} x_{1}^{-2}-\tau^{-1} x_{1}^{-3}+\frac{1}{4} \varepsilon \tau^{-2} x_{1}^{-4}-\frac{1}{4} \varepsilon x_{1}^{-2} & 0
\end{array}\right) \vec{y} .
$$

Since the system $(\mathfrak{W})^{\prime}$ has no singularity with respect to $x_{1}$, let us construct a characteristic polygon with respect to $\varepsilon$ and $\tau$. Then, the characteristic 
polygon has two vertices:

$$
\mathrm{Q}_{0}=\left(0,-\frac{1}{2}\right) \cdot \mathrm{Q}_{1}=\left(\frac{1}{2},-1\right)
$$

as is shown in figure.

We divide the domain $\left(\tilde{\mathrm{D}}_{10}\right)$ into following three subdomains :

$$
\begin{array}{rr}
\left(\mathrm{K}_{1}\right) & \left|\varepsilon \tau^{-1}\right| \leqq a_{1} ; \\
\left(\mathrm{K}_{2}\right) & a_{2} \leqq \\
\varepsilon \tau^{-1} \mid \leqq A_{2} ;
\end{array}
$$$$
\left(\mathrm{K}_{3}\right) \quad\left|\varepsilon^{-1} \tau\right| \leqq b_{3} .
$$

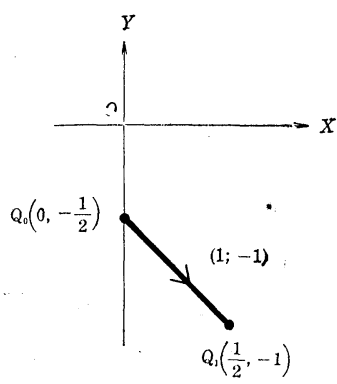

Fig. 18

If we take $b_{3}=1 / A_{2}$ and $a_{2}=a_{1}$, the union of these three subdomains covers the domain $\left(\widetilde{\mathrm{D}}_{10}\right)$ without lacuna.

\section{§53. Domain $\left(\mathbf{K}_{1}\right)$.}

In order to investigate the behavior of solutions of the system (WB)' in the subdomain $\left(\mathrm{K}_{1}\right)$, we shall make a transformation

$$
\vec{y}=\left(\begin{array}{cc}
1 & 0 \\
0 & \tau^{-\frac{1}{2}}
\end{array}\right) \vec{z}
$$

noticing that the coordinate of the vertex $Q_{0}$ is $\left(0,-\frac{1}{2}\right)$. Then, the system $(\mathfrak{B})^{\prime}$ will be changed into

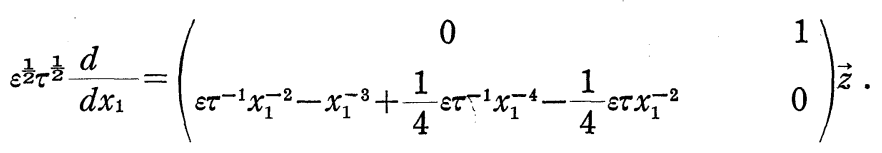

If we introduce a new parameter

$$
\varepsilon_{1}=\varepsilon \tau^{-1},
$$

the system (53.2) is written as

$$
\varepsilon_{1}^{\frac{1}{2} \tau} \frac{d \vec{z}}{d x_{1}}=\left(\begin{array}{cc}
0 & 1 \\
\varepsilon_{1} x_{1}^{-2}-x_{1}^{-3}+\frac{1}{4} \varepsilon_{1} x_{1}^{-4}-\frac{1}{4} \varepsilon_{1} \tau^{2} x_{1}^{-2} & 0
\end{array}\right) \vec{z}
$$

and the considered domain is reduced to

$$
\left|\varepsilon_{1}\right| \leqq a_{1}, \quad 0<|\tau| \leqq b_{0}, \quad c \leqq\left|x_{1}\right| \leqq C .
$$

Therefore, we see easily that the coefficient matrix of (53.4) has two distinct characteristic values in the domain $\left(\widetilde{\mathrm{K}}_{1}\right)$, since the quantity $a_{1}$ is supposed to be sufficiently small. This shows that the system (53.4) is a system of Type G if we put $\varepsilon_{1}=\alpha^{2}, \tau=\beta$ and $x_{1}=\xi$.

Thus we have the following:

XXX. In the subdomain

$$
\left|\varepsilon \tau^{-1}\right| \leqq a_{1}, \quad c \leqq\left|\tau^{-1} x\right| \leqq C,
$$

the system (W)' will be reduced to a system of Type G by a transformation 


$$
\left\{\begin{array}{l}
\vec{y}=\left(\begin{array}{cc}
1 & 0 \\
0 & \tau^{-\frac{1}{2}}
\end{array}\right) \overrightarrow{\mathfrak{w}}, \\
\varepsilon_{1}\left(=\varepsilon \tau^{-1}\right)=\alpha^{2}, \\
\tau=\beta, \\
x_{1}\left(=\tau^{-1} x\right)=\xi .
\end{array}\right.
$$

\section{§54. Domain $\left(\mathbf{K}_{2}\right)$.}

We will study the system (WB) ${ }^{\prime}$ in the domain $\left(\mathrm{K}_{2}\right)$. As is easily seen, the domain $\left(\mathrm{K}_{2}\right)$ is contained in a domain

$$
b \leqq\left|\varepsilon^{-1} \tau\right| \leqq B, \quad 0<|\varepsilon| \leqq a_{0}, \quad 0<|\tau| \leqq b_{0}, \quad 0<|x| \leqq c_{0} .
$$

This domain is equivalent to the auxiliary subdomain $\left(D_{7}\right)$, in which the behavior of the solutions has been already investigated in Chapter IV.

Thus we get the following:

XXXI. By repeating the reduction as in Chapter IV for the domain

$$
a_{2} \leqq\left|\varepsilon \tau^{-1}\right| \leqq A_{2}, \quad c \leqq\left|\tau^{-1} x\right| \leqq C,
$$

we can study the behavior of solutions of the Whittaker's differential equation in the domain $\left(\mathrm{K}_{2}\right)$. The reduced forms in this domain are of Type $\mathrm{A}, \mathrm{C}, \mathrm{E}$ and $\mathrm{F}$.

§55. Domain $\left(\mathbf{K}_{3}\right)$.

Noticing that the coordinate of the vertex $Q_{1}$ is $\left(\frac{1}{2},-1\right)$, if we make a change of variables:

$$
\left\{\begin{array}{l}
\vec{y}=\left(\begin{array}{cc}
1 & 0 \\
0 & \varepsilon^{\frac{1}{2}} \tau^{-1}
\end{array}\right) \vec{z} \\
\varepsilon^{-1} \tau=\tau_{1}
\end{array}\right.
$$

the system (W)' is transformed into

$$
\varepsilon \tau_{1} \frac{d \vec{z}}{d x_{1}}=\left(\begin{array}{cc}
0 & 1 \\
\frac{4 x_{1}^{2}-4 \tau_{1} x_{1}+1}{4 x_{1}^{4}}-\frac{1}{4} \varepsilon^{2} \tau_{1}^{2} x_{1}^{-2} & 0
\end{array}\right) \vec{z}
$$

and the domain $\left(\mathrm{K}_{3}\right)$ is reduced to

$$
0<|\varepsilon| \leqq a_{0}, \quad\left|\tau_{1}\right| \leqq b_{3}, \quad c \leqq\left|x_{1}\right| \leqq C .
$$

We see immediately that the system (55.2) has two turning points in the considered domain and such turning points are given by the two roots $f_{ \pm}\left(\tau_{1}\right)$ of an algebraic equation:

$$
4 x_{1}^{2}-4 \tau_{1} x_{1}+1=0
$$

Hence,

$$
f_{ \pm}\left(\tau_{1}\right)=\frac{1}{2}\left(\tau_{1} \pm \sqrt{\tau_{1}^{2}-1}\right) .
$$

It is easily verified that these functions do not vanish for any value of $\tau_{1}$ and 
are bounded for $\left|\tau_{1}\right| \leqq b_{3}$. Therefore, we can suppose the following inequality to be satisfied:

$$
c<\left|f_{ \pm}\left(\tau_{1}\right)\right|<C
$$

for $\left|\tau_{1}\right| \leqq b_{3}$.

Now we divide the domain $\left(\widetilde{\mathrm{K}}_{3}\right)$ into following two subdomains :

$$
\begin{aligned}
& \left|x_{1}-f_{ \pm}\left(\tau_{1}\right)\right| \geqq c_{1} ; \\
& \left|x_{1}-f_{ \pm}\left(\tau_{1}\right)\right| \leqq c_{1} .
\end{aligned}
$$

Clearly, in the subdomain $\left(\mathrm{K}_{4}\right)$ the coefficient matrix of (55.2) has two distinct characteristic values. It follows that the system (55.2) is of Type G.

Thus we have the following:

XXXII. In the subdomain

$$
\left\{\begin{array}{l}
\left|\varepsilon^{-1} \tau\right| \leqq b_{3}, \quad c \leqq\left|\tau^{-1} x\right| \leqq C, \\
\left|\tau^{-1} x-f_{ \pm}\left(\varepsilon^{-1} \tau\right)\right| \geqq c_{1}
\end{array}\right.
$$

a transformation

$$
\left\{\begin{array}{l}
\vec{y}=\left(\begin{array}{cc}
1 & 0 \\
0 & \varepsilon^{\frac{1}{2}} \tau^{-1}
\end{array}\right) \overrightarrow{\mathfrak{w}}, \\
\varepsilon=\alpha, \\
\tau_{1}\left(=\varepsilon^{-1} \tau\right)=\beta, \\
x_{1}\left(=\tau^{-1} x\right)=\xi
\end{array}\right.
$$

will reduce the system $(\mathfrak{B})^{\prime}$ to a system of Type $\mathrm{G}$.

\section{§56. Domain $\left(\mathbf{K}_{5}\right)$.}

We consider the system (55.2) in the domain $\left(\mathrm{K}_{5}\right)$. If we introduce a new independent variable

$$
x_{2}=x_{1}-f_{ \pm}\left(\tau_{1}\right)\left(=\tau^{-1} x-f_{ \pm}\left(\varepsilon^{-1} \tau\right)\right),
$$

the system (55.2) is written as

$$
\varepsilon \tau_{1} \frac{d \vec{z}}{d x_{2}}=\left(\begin{array}{cc}
0 & 1 \\
\frac{\left(2 f_{ \pm}\left(\tau_{1}\right)-\tau_{1}\right) x_{2}+x_{2}^{2}}{\left(f_{ \pm}\left(\tau_{1}\right)+x_{2}\right)^{4}}-\frac{\varepsilon^{2} \tau_{1}^{2}}{4\left(f_{ \pm}\left(\tau_{1}\right)+x_{2}\right)^{2}} & 0
\end{array}\right) \vec{z}
$$

and the considered domain $\left(\mathrm{K}_{5}\right)$ is reduced to

$$
0<|\varepsilon| \leqq a_{0}, \quad\left|\tau_{1}\right| \leqq b_{3}, \quad\left|x_{2}\right| \leqq c_{1} .
$$

By definition of $f_{ \pm}\left(\tau_{1}\right)$, we have

$$
2 f_{ \pm}\left(\tau_{1}\right)-\tau_{1}= \pm \sqrt{\tau_{1}^{2}-1}, 2 f_{ \pm}(0)= \pm i
$$

Hence, $x_{2}=0$ may possibly be a turning point. Since the coefficient matrix is regarded as a matrix function developable in a uniformly convergent triple power series of $\varepsilon, \tau_{1}$ and $x_{2}$ in the domain $\left(\widetilde{K}_{5}\right)$, we can construct a characteristic polyhedron. In this case, it is sufficient to consider a modified characteristic polyhedron since $x_{2}=0$ is a regular point. As is shown in figure, the modified characteristic polyhedron has two vertices: 


$$
\mathrm{V}_{1}=\left(0,0, \frac{1}{2}\right), \quad \mathrm{V}_{2}=\left(\frac{1}{3}, \frac{1}{3}, 0\right) .
$$

At the vertex $V_{1}$ four sides meet. Therefore, there exist four characteristic trigonal pyramids. An elementary calculation shows that the directional parameters of the three edges of such four trigonal pyramids are given by

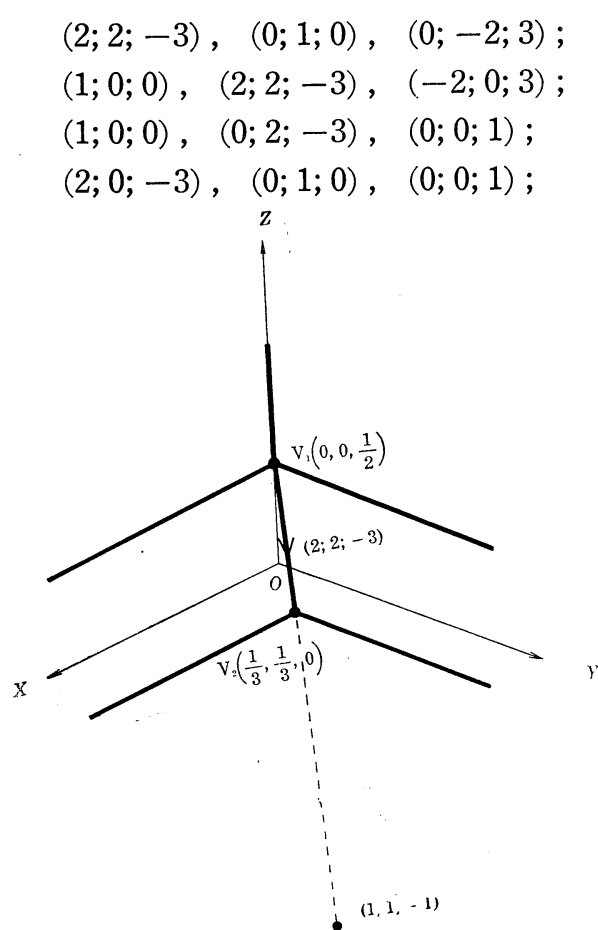

Fig. 19

$$
\left(\widetilde{\mathrm{K}}_{5}\right) \subset\left\{\begin{array}{l}
\left|\varepsilon^{-2} \tau_{1}^{-2} x_{2}^{3}\right| \leqq C_{8} \\
\left\{\begin{array}{l}
C_{8} \leqq\left|\varepsilon^{-2} \tau_{1}^{-2} x_{2}^{3}\right|, \\
C_{8} \leqq\left|\varepsilon^{-2} \tau_{1}^{-2} x_{2}^{3}\right|,
\end{array},\right.
\end{array}\right.
$$

$\mathrm{S}_{2} \cup \mathrm{S}_{3} \cup \mathrm{S}_{4}, \quad \mathrm{~S}_{1}: \quad Y=0 ;$

$\mathrm{S}_{1} \cup \mathrm{S}_{2} \cup \mathrm{S}_{3}, \quad \mathrm{~S}_{2}: \quad 3 Y+2 Z=1 ;$

$\mathrm{S}_{1} \cup \mathrm{S}_{2} \cup \mathrm{S}_{4}, \quad \mathrm{~S}_{3}: 3 X \quad+2 Z=1$;

$\mathrm{S}_{1} \cup \mathrm{S}_{3} \cup \mathrm{S}_{4}$,

$\mathrm{S}_{4}: \quad X$

$=0$.

At the vertex $V_{2}$ there exists only one characteristic trigonal pyramid whose directional parameters are

$$
(1 ; 0 ; 0),(0 ; 1 ; 0), \quad(-2 ;-2 ; 3) \text {. }
$$

Thus we have obtained five systems of the directional parameters and, consequently, we find five principal subdomains. However, we consider especially following three principal subdomains :

$\begin{array}{ccc}\left(\Omega_{6}\right) & \left|\varepsilon^{2} \tau_{1}^{2} x_{2}^{-3}\right| \leqq a_{6}, \quad\left|\tau_{1}^{-2} x_{2}^{3}\right| \leqq c_{6} ; \\ \left(\Omega_{7}\right) & \left|\tau_{1}^{2} x_{2}^{-3}\right| \leqq B_{7} ; \\ \left(\Omega_{8}\right) & \left|\varepsilon^{-2} \tau_{1}^{-2} x_{2}^{3}\right| \leqq C_{8} .\end{array}$

Then we assert that if $C_{8}=a_{6}^{-1}$

$$
\widetilde{\mathrm{K}}_{5} \subset \Re_{6} \cup \Re_{7} \cup \Re_{8} \text {. }
$$

Indeed,

$$
\begin{array}{cc}
=\left(\Re_{8}\right) ; & \\
\left|\tau_{1}^{-2} x_{2}^{3}\right| \leqq c_{6} & =\left(\Re_{6}\right) ; \\
c_{6} \leqq\left|\tau_{1}^{-2} x_{2}^{3}\right| &
\end{array}
$$

§57. Domains $\left(\Re_{6}\right),\left(\Re_{7}\right)$ and $\left(\Re_{8}\right)$.

\section{$1^{\circ}$. Domains $\left(\Omega_{6}\right)$ and $\left(\Omega_{7}\right)$.}

We shall first consider the system (56.2) in the domains $\left(\Omega_{6}\right)$ and $\left(\Omega_{7}\right)$. We should notice that these two domains correspond to the vertex $V_{1}=\left(0,0, \frac{1}{2}\right)$. Let us make a transformation

$$
\vec{z}=\left(\begin{array}{cc}
1 & 0 \\
0 & x_{2}^{\frac{1}{2}}
\end{array}\right) \vec{u}
$$

Then, the system (56.2) is changed into

$$
\left(\varepsilon \tau_{1} x_{2}^{-\frac{3}{2}}\right) \cdot x_{2} \frac{d \vec{u}}{d x_{2}}=\left(\begin{array}{cc}
0 & 1 \\
L\left(\varepsilon, \tau_{1}, x_{2}\right) & -\frac{1}{2}\left(\varepsilon \tau_{1} x_{2}^{-\frac{3}{2}}\right)
\end{array}\right) \vec{u},
$$


where

$$
L=\frac{\left(2 f_{ \pm}\left(\tau_{1}\right)-\tau_{1}\right)+x_{2}}{\left(f_{ \pm}\left(\tau_{1}\right)+x_{2}\right)^{4}}-\frac{\varepsilon^{2} \tau_{1}^{2} x_{2}^{-1}}{4\left(f_{ \pm}\left(\tau_{1}\right)+x_{2}\right)^{2}} .
$$

However, since we can write

$$
\left\{\begin{aligned}
\varepsilon \tau_{1} x_{2}^{-\frac{3}{2}} & =\left\{\begin{array}{l}
\left(\varepsilon^{2} \tau_{1}^{2} x_{2}^{-3}\right)^{\frac{1}{2}} \cdot \tau_{1}^{0} \cdot\left(\tau_{1}^{-2} x_{2}^{3}\right)^{0}, \\
\varepsilon^{1} \cdot\left(\tau_{1}^{2} x_{2}^{-3}\right)^{\frac{1}{2}} \cdot x_{2}^{0},
\end{array}\right. \\
x_{2} & =\left\{\begin{array}{l}
\tau_{1}^{\frac{2}{3}} \cdot\left(\tau_{1}^{-2} x_{2}^{3}\right)^{\frac{1}{3}} \\
x_{2},
\end{array}\right. \\
\varepsilon^{2} \tau_{1}^{2} x_{2}^{-1} & =\left\{\begin{array}{l}
\left(\varepsilon^{2} \tau_{1}^{2} x_{2}^{-3}\right) \cdot \tau_{1}^{\frac{4}{3}} \cdot\left(\tau_{1}^{-2} x_{2}^{3}\right)^{\frac{2}{3}}, \\
\varepsilon^{2} \cdot\left(\tau_{1}^{2} x_{2}^{-3}\right) \cdot x_{2}^{2},
\end{array}\right. \\
\tau_{1} & =\left\{\begin{array}{l}
\tau_{1}, \\
\left(\tau_{1}^{2} x_{2}^{-3}\right)^{\frac{1}{2}} \cdot x_{2}{ }^{\frac{3}{2}}
\end{array}\right.
\end{aligned}\right.
$$

it is easily seen that the coefficient matrix of (57.2) has two distinct characteristic values in both of the domains $\left(\Omega_{6}\right)$ and $\left(\Re_{7}\right)$, whence follows immediately that the system (57.2) is a system of Type A if we put $\varepsilon=\alpha^{6}, \tau_{1}=\beta^{6}, x_{2}=\xi^{6}$.

Thus we have established the following:

XXXIII. In the subdomain

$$
\left\{\begin{array}{l}
\left|\varepsilon^{-1} \tau\right| \leqq b_{3}, \quad\left|\tau^{-1} x-f_{ \pm}\left(\varepsilon^{-1} \tau\right)\right| \leqq c_{1} \\
\left|\tau^{2}\left\{\tau^{-1} x-f_{ \pm}\left(\varepsilon^{-1} \tau\right)\right\}^{-3}\right| \leqq a_{6}, \quad\left|\left(\varepsilon^{-1} \tau\right)^{-2}\left(\tau^{-1} x-f_{ \pm}\left(\varepsilon^{-1} \tau\right)\right)^{3}\right| \leqq c_{6}
\end{array}\right.
$$

or

$$
\left\{\begin{array}{l}
\left|\varepsilon^{-1} \tau\right| \leqq b_{3}, \quad\left|\tau^{-1} x-f_{ \pm}\left(\varepsilon^{-1} \tau\right)\right| \leqq c_{1} \\
\left|\left(\varepsilon^{-1} \tau\right)^{2}\left(\tau^{-1} x-f_{ \pm}\left(\varepsilon^{-1} \tau\right)\right)^{-3}\right| \leqq B_{7}
\end{array}\right.
$$

the system (W)' will be reduced to a system of Type A by a transformation

$$
\left\{\begin{array}{l}
\vec{y}=\left(\begin{array}{cc}
1 & 0 \\
0 & \varepsilon^{\frac{1}{2}} \tau^{-1} \cdot x_{2}^{\frac{1}{2}}
\end{array}\right) \overrightarrow{\mathfrak{w}} \\
\varepsilon=\alpha^{6} \\
\tau_{1}\left(=\varepsilon^{-1} \tau\right)=\beta^{6} \\
x_{2}\left(=x_{1}-f_{ \pm}\left(\tau_{1}\right)=\tau^{-1} x-f_{ \pm}\left(\varepsilon^{-1} \tau\right)\right)=\xi^{6}
\end{array}\right.
$$

$\mathbf{2}^{\circ}$. Domain $\left(\AA_{8}\right)$.

We consider the system $(56.2)$ in the domain $\left(\Omega_{8}\right)$. Noticing that the coordinate of the vertex $\mathrm{V}_{2}$ is $\left(\frac{1}{3}, \frac{1}{3}, 0\right)$, let us make a transformation

$$
\vec{z}=\left(\begin{array}{cc}
1 & 0 \\
0 & \varepsilon^{\frac{1}{3}} \tau_{1}^{\frac{1}{3}}
\end{array}\right) \vec{u}
$$

Then, the system satisfied by $\vec{u}$ is easily found to be

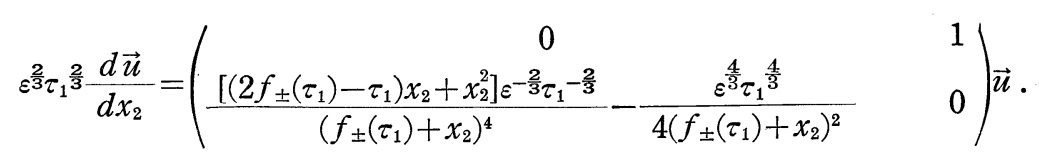


If we introduce a new independent variable

$$
x_{3}=\varepsilon^{-\frac{2}{3}} \tau_{1}-\frac{2}{3} x_{2},
$$

the considered domain becomes

$$
0<|\varepsilon| \leqq a_{0}, \quad\left|\tau_{1}\right| \leqq b_{3}, \quad\left|x_{3}\right| \leqq C_{8^{\frac{1}{3}}}
$$

and the system (57.4) is reduced to

$$
\frac{d \vec{u}}{d x_{3}}=\left(\begin{array}{cc}
0 & 1 \\
\frac{\left(2 f_{ \pm}\left(\tau_{1}\right)-\tau_{1}\right) x_{3}+\varepsilon^{\frac{2}{3}} \tau_{1}^{\frac{2}{3}} x_{3}^{2}}{\left(f_{ \pm}\left(\tau_{1}\right)+\varepsilon^{\frac{2}{3}} \tau_{1}^{\frac{2}{3}} x_{3}\right)^{4}}-\frac{\varepsilon^{\frac{4}{3}} \tau_{1}^{\frac{4}{3}}}{4\left(f_{ \pm}\left(\tau_{1}\right)+\varepsilon^{\frac{2}{3}} \tau_{1}^{\frac{2}{3}} x_{3}\right)^{2}} & 0
\end{array}\right) \vec{u} .
$$

From this we can conclude that the system (57.6) (where we change $\varepsilon, \tau_{1}, x_{3}$ into $\left.\alpha^{3}, \beta^{3}, \xi\right)$ is a system of Type F.

Hence, we have the following:

XXXIV. In the subdomain

$$
\left\{\begin{array}{l}
\left|\varepsilon^{-1} \tau\right| \leqq b_{3}, \quad\left|\tau^{-1} x-f_{ \pm}\left(\varepsilon^{-1} \tau\right)\right| \leqq c_{1} \\
\left|\tau^{-2}\left(\tau^{-1} x-f_{ \pm}\left(\varepsilon^{-1} \tau\right)\right)^{3}\right| \leqq C_{8}
\end{array}\right.
$$

a transformation

$$
\left\{\begin{aligned}
& \vec{y}=\left(\begin{array}{cc}
1 & 0 \\
0 & \varepsilon^{\frac{1}{2}} \tau^{-1} \cdot \varepsilon^{\frac{1}{3}} \tau_{1}^{\frac{1}{3}}
\end{array}\right) \overrightarrow{\mathfrak{w}} \\
& \varepsilon=\alpha^{3} \\
& \tau_{1}(\left.=\varepsilon^{-1} \tau\right)=\beta^{3} \\
& x_{3}\left(=\varepsilon^{-\frac{2}{3}} \tau_{1}^{-\frac{2}{3}} x_{2}=\tau^{-\frac{2}{3}}\left(x_{1}-f_{ \pm}\left(\tau_{1}\right)\right)=\tau^{-\frac{2}{3}}\left(\tau^{-1} x-f_{ \pm}\left(\varepsilon^{-1} \tau\right)\right)=\xi\right.
\end{aligned}\right.
$$

will reduce the system (W)' to a system of Type $\mathrm{F}$.

\section{$\S 58$. Conclusion.}

Summarizing the discussions done in this chapter, we get the following:

Theorem 4. If we divide the auxiliary subdomain

$$
c_{10} \leqq\left|\tau^{-1} x\right| \leqq C_{10}, \quad 0<|\varepsilon| \leqq a_{0}, \quad 0<|\tau| \leqq b_{0}, \quad 0<|x| \leqq c_{0}
$$

into six subdomains $\mathrm{K}_{1}, \mathrm{~K}_{2}, \mathrm{~K}_{4}, \Re_{6}, \Re_{7}$ and $\Re_{8}$ in a way:

$$
\begin{aligned}
\mathrm{D}_{10} & =\mathrm{K}_{1} \cup \mathrm{K}_{2} \cup \mathrm{K}_{3}, \\
\mathrm{~K}_{3} & =\mathrm{K}_{4} \cup \mathrm{K}_{5} \\
\mathrm{~K}_{5} & \subset \Re_{6} \cup \Re_{7} \cup \Re_{8},
\end{aligned}
$$

we can obtain the reduced form in each of these subdomains. The types of such reduced forms are given by a following table:

\begin{tabular}{l|c|c|c|c|c|c}
\hline Subdomain & $\mathrm{K}_{1}$ & $\mathrm{~K}_{2}$ & $\mathrm{~K}_{4}$ & $\Re_{6}$ & $\Re_{7}$ & $\Re_{8}$ \\
\cline { 2 - 7 } Normal form & $\mathrm{G}$ & $\mathrm{A}, \mathrm{C}, \mathrm{E}, \mathrm{F}$ & $\mathrm{G}$ & $\mathrm{A}$ & $\mathrm{A}$ & $\mathrm{F}$ \\
\hline Proposition & $\mathrm{XXX}$ & $\mathrm{XXXI}$ & $\mathrm{XXXII}$ & $\mathrm{XXXIII}$ & $\mathrm{XXXIII}$ & $\mathrm{XXXIV}$ \\
\hline Section & 53 & 54 & 55 & 57 & 57 & 57 \\
\hline
\end{tabular}




\section{Chapter VIII. Study of the behavior of the solutions in the auxiliary subdomain $\left(D_{11}\right)$.}

We will show that we can divide the subdomain $\left(D_{11}\right)$ into a finite number of subdomains so that the reduced form in each of those subdomains takes one of Types A, C, E, F and G.

\section{§59. Auxiliary subdomain $\left(\mathbf{D}_{11}\right)$.}

This domain is written as $\left(D_{11}\right)$

$$
c \leqq\left|\varepsilon \tau^{-2} x\right| \leqq C, \quad 0<|\varepsilon| \leqq a_{0}, \quad 0<|\tau| \leqq b_{0}, \quad 0<|x| \leqq c_{0} .
$$

If we introduce a new independent variable

$$
x_{1}=\varepsilon \tau^{-2} x,
$$

the considered system (WB) will be changed into

$$
\varepsilon^{\frac{3}{2} \tau^{-1}} \frac{d \vec{y}}{d x_{1}}=\left(\begin{array}{cc}
0 & 1 \\
\varepsilon^{3} \tau^{-4} x_{1}^{-2}-\varepsilon^{3} \tau^{-4} x_{1}^{-3}+\frac{1}{4} \varepsilon^{5} \tau^{-6} x_{1}^{-4}-\frac{1}{4} \varepsilon^{3} \tau^{-2} x_{1}^{-2} & 0
\end{array}\right) \vec{y}
$$

and the domain $\left(D_{11}\right)$ is reduced to $\left(\widetilde{\mathrm{D}}_{11}\right) \quad 0<|\varepsilon| \leqq a_{0}, \quad 0<|\tau| \leqq b_{0}, \quad c \leqq\left|x_{1}\right| \leqq C$.

Since the system $(\mathfrak{W})^{\prime}$ has no singularity with respect to $x_{1}$, we construct a characteristic polygon with respect to $\varepsilon$ and $\tau$. Then, as is shown in figure, the characteristic polygon has two vertices:

$$
\mathrm{Q}_{0}=\left(\frac{3}{2},-2\right), \quad \mathrm{Q}_{1}=\left(\frac{5}{2},-3\right) \text {. }
$$

We divide the domain $\left(\widetilde{\mathrm{D}}_{11}\right)$ into following three subdomains :

$$
\begin{array}{rlr}
\left|\varepsilon \tau^{-1}\right| \leqq a_{1} ; & \\
a_{2} \leqq\left|\varepsilon \tau^{-1}\right| \leqq A_{2} & & \left(a_{2}=a_{1}\right) ; \\
\left|\varepsilon^{-1} \tau\right| \leqq b_{3} & & \left(b_{3}=1 / A_{2}\right) .
\end{array}
$$

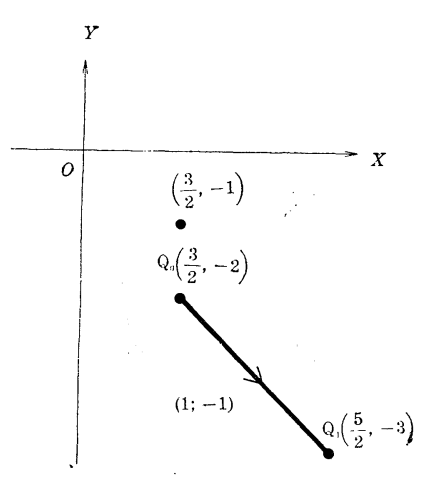

Fig. 20

$\S 60$. Domain $\left(\mathbf{L}_{2}\right)$.

The domain $\left(\mathrm{L}_{2}\right)$ is contained in the auxiliary subdomain $\left(\mathrm{D}_{7}\right)$ and the behavior of the solutions in such a domain has been already studied in Chapter IV.

Hence we have immediately the following:

XXXV. If we divide further the domain

$$
a_{2} \leqq\left|\varepsilon \tau^{-1}\right| \leqq A_{2}, \quad c \leqq\left|\varepsilon \tau^{-2} x\right| \leqq C
$$

into a finite number of subdomains in a suitable way, then we shall arrive at one of the reduced forms of Type A, C, E and F in each of such subdomains. 


\section{§ 61. Domain $\left(\mathbf{L}_{3}\right)$.}

In order to study the behavior of solutions of the system (WB) ${ }^{\prime}$ in the domain $\left(\mathrm{L}_{3}\right)$, we make a transformation

$$
\left\{\begin{aligned}
\vec{y} & =\left(\begin{array}{cc}
1 & 0 \\
0 & \varepsilon^{\frac{5}{2}} \tau^{-3}
\end{array}\right) \vec{z}, \\
\varepsilon^{-1} \tau & =\tau_{1}
\end{aligned}\right.
$$

noticing that the coordinate of the vertex $Q_{1}$ is $\left(\frac{5}{2},-3\right)$. Then, the system satisfied by $\vec{z}$ is easily found to be

$$
\varepsilon \tau_{1}^{2} \frac{d \vec{z}}{d x_{1}}=\left(\begin{array}{cc}
0 & 1 \\
\tau_{1}^{2}\left(x_{1}^{-2}-x_{1}^{-3}\right)+\frac{1}{4} x_{1}^{-4}-\frac{1}{4} \varepsilon^{2} \tau_{1}^{4} x_{1}^{-2} & 0
\end{array}\right) \vec{z}
$$

and the considered domain is reduced to

$$
0<|\varepsilon| \leqq a_{0}, \quad\left|\tau_{1}\right| \leqq b_{3}, \quad c \leqq\left|x_{1}\right| \leqq C .
$$

Since $b_{3}$ is supposed to be sufficiently small, the coefficient matrix of (61.2) has two distinct characteristic values in the domain $\left(\tilde{\mathrm{L}}_{3}\right)$ and they tend to $\pm 1 / 2 x_{1}^{2}$ as $\varepsilon$ and $\tau_{1}$ tend to 0 . Therefore, it is easily seen that the system (61.2) is a system of Type G if we put $\varepsilon=\alpha, \tau_{1}=\beta$ and $x_{1}=\xi$.

Thus we have proved the following:

XXXVI. In the subdomain

$$
\left|\varepsilon^{-1} \tau\right| \leqq b_{3}, \quad c \leqq\left|\varepsilon \tau^{-2} x\right| \leqq C,
$$

the system (W)' is reduced to a system of Type $\mathrm{G}$ by a transformation

$$
\left\{\begin{array}{l}
\vec{y}=\left(\begin{array}{cc}
1 & 0 \\
0 & \varepsilon^{\frac{5}{2}} \tau^{-3}
\end{array}\right) \overrightarrow{\mathfrak{w}}, \\
\varepsilon=\alpha, \\
\tau_{1}\left(=\varepsilon^{-1} \tau\right)=\beta, \\
x_{1}\left(=\varepsilon \tau^{-2} x\right)=\xi
\end{array}\right.
$$

§62. Domain $\left(\mathbf{L}_{1}\right)$.

Noticing that the coordinate of the vertex $Q_{0}$ is $\left(\frac{3}{2},-2\right)$, let us make a transformation

$$
\left\{\begin{aligned}
y & =\left(\begin{array}{cc}
1 & 0 \\
0 & \varepsilon^{\frac{3}{2} \tau^{-2}}
\end{array}\right) \vec{z} \\
\varepsilon \tau^{-1} & =\varepsilon_{1}
\end{aligned}\right.
$$

Then, we can derive from (WB)' the following:

$$
\tau \frac{d \vec{z}}{d x_{1}}=\left(\begin{array}{cc}
0 & 1 \\
x_{1}^{-2}-x_{1}^{-3}+\frac{1}{4} \varepsilon_{1}^{2} x_{1}^{-4}-\frac{1}{4} \tau^{2} x_{1}^{-2} & 0
\end{array}\right) \vec{z}
$$

and the considered domain is reduced to 


$$
\left|\varepsilon_{1}\right| \leqq a_{1}, \quad 0<|\tau| \leqq b_{0}, \quad c \leqq\left|x_{1}\right| \leqq C .
$$

It is easily seen that, if $x_{1} \neq 1$, the coefficient matrix of (62.2) has two distinct characteristic values in the domain $\left(\tilde{\mathrm{L}}_{1}\right)$, whence follows that the system (62.2) is of Type E.

Keeping this fact in mind, we divide the domain $\left(\tilde{\mathrm{L}}_{1}\right)$ into following two subdomains :

$$
\begin{aligned}
& \left|x_{1}-1\right| \geqq c_{1} ; \\
& \left|x_{1}-1\right| \leqq c_{1} .
\end{aligned}
$$

Then we have immediately the following:

XXXVII. In the subdomain

$$
\left\{\begin{array}{l}
\left|\varepsilon \tau^{-1}\right| \leqq a_{1}, \quad c \leqq\left|\varepsilon \tau^{-2} x\right| \leqq C, \\
\left|\varepsilon \tau^{-2} x-1\right| \geqq c_{1}
\end{array}\right.
$$

\section{a transformation}

$$
\left\{\begin{array}{l}
\vec{y}=\left(\begin{array}{cc}
1 & 0 \\
0 & \varepsilon^{\frac{3}{2}} \tau^{-2}
\end{array}\right) \overrightarrow{\mathfrak{w}}, \\
\varepsilon_{1}\left(=\varepsilon \tau^{-1}\right)=\beta, \\
\tau=\alpha, \\
x_{1}\left(=\varepsilon \tau^{-2} x\right)=\xi
\end{array}\right.
$$

changes the system (W)' into a system of Type $\mathrm{E}$.

§63. Domain $\left(\mathbf{L}_{5}\right)$.

In order to study the system (62.2) in the domain $\left(\mathrm{L}_{5}\right)$, if we introduce a new independent variable

$$
x_{2}=x_{1}-1
$$

the considered domain is reduced to

$$
\left|\varepsilon_{1}\right| \leqq a_{1}, \quad 0<|\tau| \leqq b_{0}, \quad\left|x_{2}\right| \leqq c_{1}
$$

and the systen (62.2) is written as

$$
\tau \frac{d \vec{z}}{d x_{2}}=\left(\begin{array}{cc}
0 & 1 \\
\frac{\varepsilon_{1}^{2}}{4\left(1+x_{2}\right)^{4}}+\frac{x_{2}}{\left(1+x_{2}\right)^{3}}-\frac{\tau^{2}}{4\left(1+x_{2}\right)^{2}} & 0
\end{array}\right) \vec{z} .
$$

Since $x_{2}=0$ may possibly be a turning point and the system (63.2) has no singularity with respect to $x_{2}$, let us construct a modified characteristic polyhedron. As is shown in figure, the modified characteristic polyhedron has three vertices:

$$
\mathrm{V}_{1}=\left(0,0, \frac{1}{2}\right), \quad \mathrm{V}_{2}=(1,0,0), \quad \mathrm{V}_{3}=\left(0, \frac{1}{3}, 0\right)
$$

As is shown in the figure, at each of the vertices $V_{1}, V_{2}, V_{3}$, there exists only one characteristic trigonal pyramid. The directional parameters of the three edges of such three trigonal pyramids are easily found to be 


$$
\begin{array}{lll}
(2 ; 0 ;-1), & (0 ; 2 ;-3), & (0 ; 0 ; 1) ; \\
(1 ; 0 ; 0), & (-3 ; 1 ; 0), & (-2 ; 0 ; 1) ; \\
(3 ;-1 ; 0), & (0 ; 1 ; 0), & (0 ;-2 ; 3) ;
\end{array}
$$

which correspond to $V_{1}, V_{2}, V_{3}$ respectively.

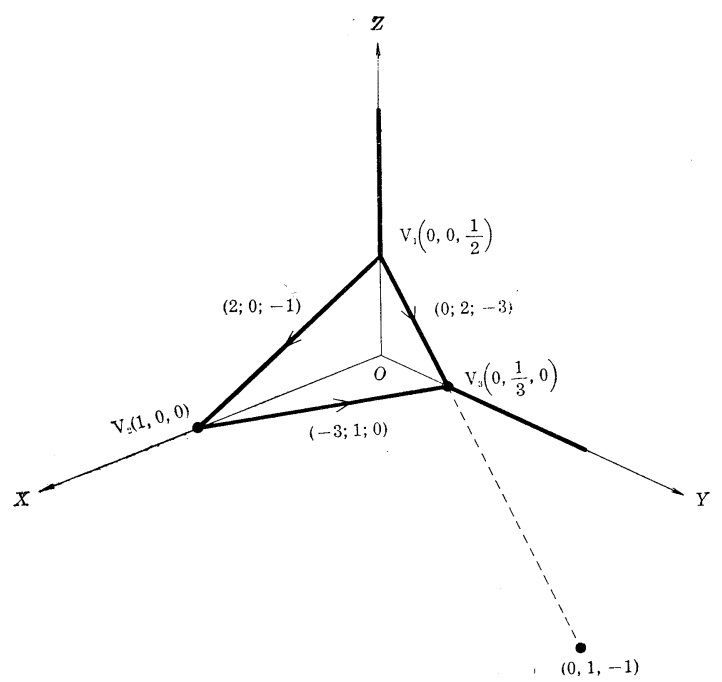

Fig. 21

Thus there exist three principal subdomains in all:

$$
\begin{array}{lll}
\left|\varepsilon_{1}^{2} x_{2}^{-1}\right| \leqq a_{6}, & \left|\tau^{2} x_{2}^{-3}\right| \leqq b_{6} ; & \\
& \left|\varepsilon_{1}^{-3} \tau\right| \leqq B_{7}, & \left|\varepsilon_{1}^{-2} x_{2}\right| \leqq c_{7} ; \\
\left|\varepsilon_{1}^{3} \tau^{-1}\right| \leqq A_{8}, & & \left|\tau^{-2} x_{2}^{3}\right| \leqq C_{8} .
\end{array}
$$

Moreover, we add a following auxiliary subdomain:

$$
c_{9} \leqq\left|\varepsilon_{1}^{-2} x_{2}\right| \leqq C_{9} \text {. }
$$

Then, the union of these four subdomains covers the domain $\left(\tilde{\mathrm{L}}_{5}\right)$ without lacuna in a following way:

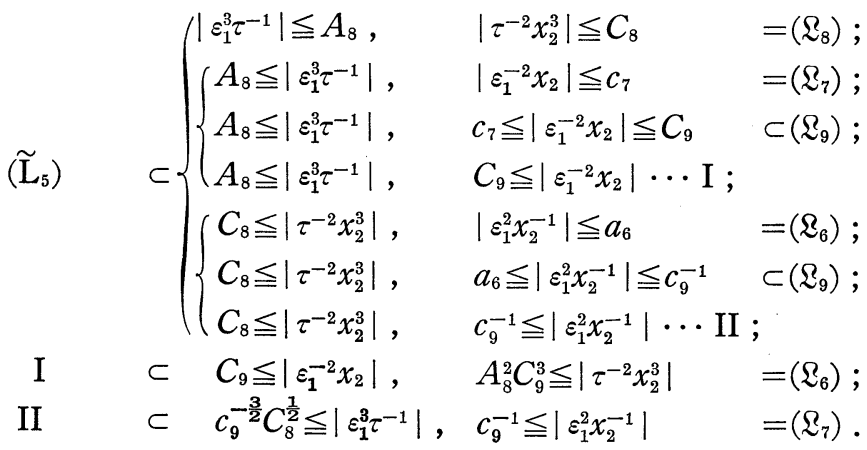

§64. Principal subdomains $\left(\mathfrak{\Omega}_{6}\right),\left(\mathfrak{l}_{7}\right),\left(\mathfrak{\Omega}_{8}\right)$.

In order to obtain the reduced form in each of these three principal sub- 
domains, we must make a further reduction of the system (63.2). The process of such a reduction is quite similar to that given in Chapter III. Hence we shall state here only the results.

XXXVIII. In the subdomain

$$
\left\{\begin{array}{l}
\left|\varepsilon \tau^{-1}\right| \leqq a_{1}, \quad\left|\varepsilon \tau^{-2} x-1\right| \leqq c_{1} \\
\left|\left(\varepsilon \tau^{-1}\right)^{2}\left(\varepsilon \tau^{-2} x-1\right)^{-1}\right| \leqq a_{6}, \quad\left|\tau^{2}\left(\varepsilon \tau^{-2} x-1\right)^{-3}\right| \leqq b_{6}
\end{array}\right.
$$

a transformation

$$
\left\{\begin{array}{l}
\vec{y}=\left(\begin{array}{cc}
1 & 0 \\
0 & \varepsilon^{\frac{3}{2}} \tau^{-2} \cdot x_{2}^{\frac{1}{2}}
\end{array}\right) \overrightarrow{\mathfrak{w}} \\
\varepsilon_{1}\left(=\varepsilon \tau^{-1}\right)=\alpha^{2} \\
\tau=\beta^{2} \\
x_{2}\left(=x_{1}-1=\varepsilon \tau^{-2} x-1\right)=\xi^{2}
\end{array}\right.
$$

reduces the system (W)' to a system of Type A.4)

XXXIX. In the subdomain

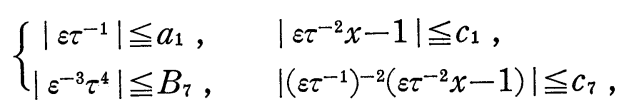

the system (W)' will be transformed into a system of Type $\mathrm{E}$ by a change of variables:

$$
\left\{\begin{array}{l}
\vec{y}=\left(\begin{array}{cc}
1 & 0 \\
0 & \varepsilon^{\frac{3}{2}} \tau^{-2} \cdot \varepsilon_{1}
\end{array}\right) \overrightarrow{\mathfrak{w}}, \\
\varepsilon_{1}\left(=\varepsilon \tau^{-1}\right)=\beta, \\
\tau_{1}\left(=\varepsilon_{1}^{-3} \tau=\varepsilon^{-3} \tau^{4}\right)=\alpha, \\
x_{3}\left(=\varepsilon_{1}^{-2} x_{2}=\varepsilon_{1}^{-2}\left(x_{1}-1\right)=\left(\varepsilon \tau^{-1}\right)^{-2}\left(\varepsilon \tau^{-2} x-1\right)\right)=\xi .
\end{array}\right.
$$

XL. In the subdomain

$$
\begin{cases}\left|\varepsilon \tau^{-1}\right| \leqq a_{1}, & \left|\varepsilon \tau^{-2} x-1\right| \leqq c_{1}, \\ \left|\varepsilon^{3} \tau^{-4}\right| \leqq A_{8}, & \left|\tau^{-2}\left(\varepsilon \tau^{-2} x-1\right)^{3}\right| \leqq C_{8},\end{cases}
$$

the system of Type $\mathrm{F}$ will be obtained if we put

$$
\left\{\begin{array}{l}
\vec{y}=\left(\begin{array}{cc}
1 & 0 \\
0 & \varepsilon^{\frac{3}{2}} \tau^{-2} \cdot \tau^{\frac{1}{3}}
\end{array}\right) \overrightarrow{\mathfrak{w}}, \\
\varepsilon_{2}\left(=\varepsilon_{1}^{3} \tau^{-1}=\varepsilon^{3} \tau^{-4}\right)=\alpha^{3}, \\
\tau=\beta^{3}, \\
x_{3}\left(=\tau^{-\frac{2}{3}} x_{2}=\tau^{-\frac{2}{3}}\left(\varepsilon \tau^{-2} x-1\right)\right)=\xi .
\end{array}\right.
$$

$\S 65$. Domain $\left(\boldsymbol{\Omega}_{9}\right)$.

$1^{\circ}$. Domain $\left(\Omega_{9}\right)$.

In order to study the system (63.2) in the auxiliary subdomain $\left(\mathbb{R}_{9}\right)$, we introduce a new independent variable:

4) In this case, in order to obtain a system of Type A, we must exchange the rôle of $\left(\varepsilon_{1}^{2} x_{2}^{-1}\right)$ and $\left(\tau^{2} x_{2}^{-3}\right)$. 
(65.1)

$$
x_{3}=\varepsilon_{1}^{-2} x_{2} .
$$

Then, the domain $\left(\Omega_{9}\right)$ will be reduced to

$$
\left|\varepsilon_{1}\right| \leqq a_{1}, \quad 0<|\tau| \leqq b_{0},
$$$$
c_{9} \leqq\left|x_{3}\right| \leqq C_{9}
$$

and the system (63.2) is transformed into

$$
\varepsilon_{1}^{-2} \tau \frac{d \vec{z}}{d x_{3}}=\left(\begin{array}{cc}
0 & 1 \\
\frac{\varepsilon_{1}^{2}}{4\left(1+\varepsilon_{1}^{2} x_{3}\right)^{4}}+\frac{\varepsilon_{1}^{2} x_{3}}{\left(1+\varepsilon_{1}^{2} x_{3}\right)^{3}}-\frac{\tau^{2}}{4\left(1+\varepsilon_{1}^{2} x_{3}\right)^{2}} & 0
\end{array}\right) \vec{z}
$$

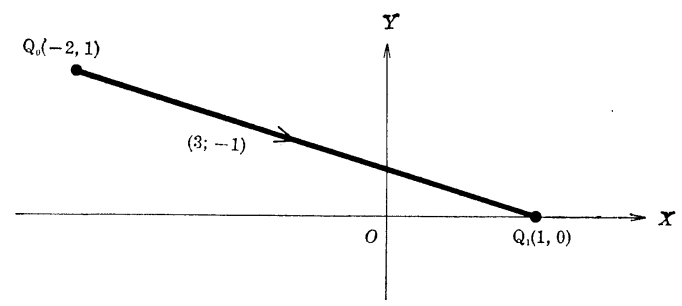

Fig. 22

It is easily seen that a characteristic polygon with respect to $\varepsilon_{1}$ and $\tau$ has two vertices:

$$
\mathrm{Q}_{0}=(-2,1), \quad \mathrm{Q}_{1}=(1,0)
$$

as is shown in figure.

We divide the domain $\left(\widetilde{\mathfrak{L}}_{9}\right)$ into following two subdomains:

$\left(\mathrm{L}_{10}\right)$

$\left(\mathrm{L}_{11}\right)$

$$
\begin{aligned}
& \left|\varepsilon_{1}^{3} \tau^{-1}\right| \leqq A_{10} ; \\
& \left|\varepsilon_{1}^{-3} \tau\right| \leqq b_{11} \quad\left(b_{11}=1 / A_{10}\right) .
\end{aligned}
$$

Then we can easily verify the following:

XLI. In the subdomain

$\left(\mathrm{L}_{10}\right)$

$$
\left\{\begin{array}{l}
\left|\varepsilon \tau^{-1}\right| \leqq a_{1}, \quad\left|\varepsilon \tau^{-2} x-1\right| \leqq c_{1} \\
c_{9} \leqq\left|\left(\varepsilon \tau^{-1}\right)^{-2}\left(\varepsilon \tau^{-2} x-1\right)\right| \leqq C_{9} \\
\left|\varepsilon^{3} \tau^{-4}\right| \leqq A_{10}
\end{array}\right.
$$

the system (W)' is reduced to a system of Type $\mathrm{F}$ if we apply following two transformations successively:

$$
\left\{\begin{array}{l}
\vec{y}=\left(\begin{array}{cc}
1 & 0 \\
0 & \varepsilon^{\frac{3}{2}} \tau^{-2}
\end{array}\right) \vec{z}, \quad \vec{z}=\left(\begin{array}{cc}
1 & 0 \\
0 & \varepsilon_{1}^{-2} \tau
\end{array}\right) \overrightarrow{\mathfrak{w}}, \\
\varepsilon_{2}\left(=\varepsilon_{1}^{3} \tau^{-1}=\varepsilon^{3} \tau^{-4}\right)=\alpha^{3}, \\
\tau=\beta^{3}, \\
x_{3}\left(=\varepsilon_{1}^{-2} x_{2}=\left(\varepsilon \tau^{-1}\right)^{-2}\left(\varepsilon \tau^{-2} x-1\right)\right)=\xi .
\end{array}\right.
$$

$2^{\circ}$. Domain $\left(\mathbf{L}_{11}\right)$.

In order to study the system $(65.2)$ in the domain $\left(L_{11}\right)$, let us make a transformation

$$
\left\{\begin{array}{l}
\vec{z}=\left(\begin{array}{ll}
1 & 0 \\
0 & \varepsilon_{1}
\end{array}\right) \vec{u} \\
\varepsilon_{1}^{-3} \tau=\tau_{1}
\end{array}\right.
$$

noticing that the coordinate of the vertex $Q_{1}$ is $(1,0)$. Then the system (65.2) will be reduced to

$$
\tau_{1} \frac{d \vec{u}}{d x_{3}}=\left(\begin{array}{cc}
0 & 1 \\
\frac{4 \varepsilon_{1}^{2} x_{3}^{2}+4 x_{3}+1}{4\left(1+\varepsilon_{1}^{2} x_{3}\right)^{4}}-\frac{\varepsilon_{1}^{4} \tau_{1}^{2}}{4\left(1+\varepsilon_{1}^{2} x_{3}\right)^{2}} & 0
\end{array}\right) \vec{\imath}
$$


and the domain $\left(\mathrm{L}_{11}\right)$ is written as

$$
\left|\varepsilon_{1}\right| \leqq a_{1}, \quad\left|\tau_{1}\right| \leqq b_{11}
$$$$
c_{9} \leqq\left|x_{3}\right| \leqq C_{9} .
$$

Let $f_{ \pm}\left(\varepsilon_{1}\right)$ be two roots of an algebraic equation

i.e.

$$
4 \varepsilon_{1}^{2} x_{3}^{2}+4 x_{3}+1=0 \text {, }
$$

$$
f_{ \pm}\left(\varepsilon_{1}\right)=\frac{-1 \pm \sqrt{1-\varepsilon_{1}^{2}}}{2 \varepsilon_{1}^{2}}
$$

Then, we see immediately that the points $x_{3}=f_{ \pm}\left(\varepsilon_{1}\right)$ may possibly be turning points. However, as $\varepsilon_{1}$ tends to 0 , we have

$$
f_{+}\left(\varepsilon_{1}\right) \rightarrow-\frac{1}{4}, \quad f_{-}\left(\varepsilon_{1}\right) \rightarrow-\frac{1}{\varepsilon_{1}^{2}} \rightarrow \infty .
$$

Hence the turning point contained in the domain $\left(\widetilde{\mathrm{L}}_{11}\right)$ is given by $x_{3}=f_{+}\left(\varepsilon_{1}\right)$.

Keeping this fact in mind, we divide the domain $\left(\widetilde{\mathrm{L}}_{11}\right)$ into following two subdomains
$\left(\mathrm{L}_{12}\right)$

$$
\left|x_{3}-f_{+}\left(\varepsilon_{1}\right)\right| \geqq c_{2} ;
$$$$
\left|x_{3}-f_{+}\left(\varepsilon_{1}\right)\right| \leqq c_{2} \text {. }
$$

Then we have at once the following:

XLII. In the subdomain

$\left(\mathrm{L}_{12}\right)$

$$
\left\{\begin{array}{l}
\left|\varepsilon \tau^{-1}\right| \leqq a_{1}, \quad\left|\varepsilon \tau^{-2} x-1\right| \leqq c_{1}, \\
c_{9} \leqq\left|\left(\varepsilon \tau^{-1}\right)^{-2}\left(\varepsilon \tau^{-2} x-1\right)\right| \leqq C_{9}, \\
\left|\varepsilon^{-3} \tau^{4}\right| \leqq b_{11}, \\
\left|\left(\varepsilon \tau^{-1}\right)^{-2}\left(\varepsilon \tau^{-2} x-1\right)-f_{+}\left(\varepsilon \tau^{-1}\right)\right| \leqq c_{2},
\end{array}\right.
$$

a substitution

$$
\left\{\begin{array}{l}
\vec{y}=\left(\begin{array}{cc}
1 & 0 \\
0 & \varepsilon^{\frac{3}{2} \tau^{-2} \cdot \varepsilon_{1}}
\end{array}\right) \overrightarrow{\mathfrak{w}}, \\
\varepsilon_{1}\left(=\varepsilon \tau^{-1}\right)=\beta, \\
\tau_{1}\left(=\varepsilon_{1}^{-3} \tau=\varepsilon^{-3} \tau^{4}\right)=\alpha, \\
x_{3}\left(=\varepsilon_{1}^{-2} x_{2}=\left(\varepsilon \tau^{-1}\right)^{-2}\left(\varepsilon \tau^{-2} x-1\right)\right)=\xi
\end{array}\right.
$$

will yield a system of Type $\mathrm{E}$.

$3^{\circ}$. Domain $\left(\mathbf{L}_{13}\right)$.

In order to study the system $(65.4)$ in the domain $\left(\mathrm{L}_{18}\right)$, we introduce a new independent variable:

$$
x_{4}=x_{3}-f_{+}\left(\varepsilon_{1}\right) \text {. }
$$

Then the domain $\left(\mathrm{L}_{18}\right)$ is reduced to

$$
\left|\varepsilon_{1}\right| \leqq a_{1}, \quad\left|\tau_{1}\right| \leqq b_{11}, \quad\left|x_{4}\right| \leqq c_{2}
$$

and the system (65.4) will be changed into

$$
\tau_{1} \frac{d \vec{u}}{d x_{4}}=\left(\frac{\varepsilon_{1}^{2} x_{4}^{2}+\left(1+2 \varepsilon_{1}^{2} f_{+}\left(\varepsilon_{1}\right)\right) x_{4}}{\left(1+\varepsilon_{1}^{2} f_{+}\left(\varepsilon_{1}\right)+\varepsilon_{1}^{2} x_{4}\right)^{4}}-\frac{\varepsilon_{1}^{4} \tau_{1}^{2}}{4\left(1+\varepsilon_{1}^{2} f_{+}\left(\varepsilon_{1}\right)+\varepsilon_{1}^{2} x_{4}\right)^{2}} \quad 10\right) \vec{u}
$$




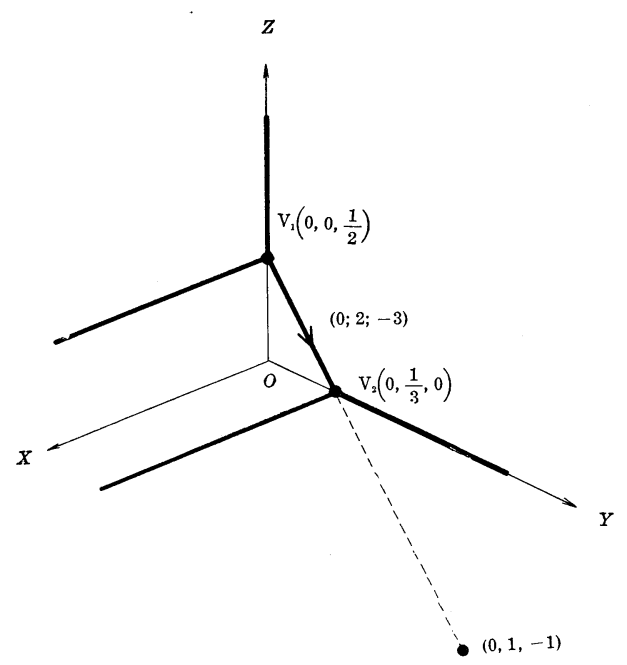

Fig. 23

Let us construct a modified characteristic polyhedron. As is shown in figure, the modified characteristic polyhedron has two vertices:

$$
\mathrm{V}_{1}=\left(0,0, \frac{1}{2}\right), \quad \mathrm{V}_{2}=\left(0, \frac{1}{3}, 0\right)
$$

and has the form of a trigonal cylinder with its base on $(Y, Z)$-plane.

We divide the domain $\left(\tilde{\mathrm{L}}_{13}\right)$ into following two subdomains :

$\left(\Omega_{14}\right) \quad\left|\tau_{1} x_{4}^{-\frac{3}{2}}\right| \leqq b_{14} ;$

$$
\left|\tau_{1}^{-\frac{2}{3}} x_{4}\right| \leqq C_{15}\left(C_{15}=b_{14}^{-\frac{2}{3}}\right) \text {. }
$$

Then, by the same reasonings as in $\S \S 42,43$, we can easily prove the followings :

XLIII. In the subdomain

$\left(\Omega_{14}\right)$

$$
\left\{\begin{array}{l}
\left|\varepsilon \tau^{-1}\right| \leqq a_{1}, \quad\left|\varepsilon \tau^{-2} x-1\right| \leqq c_{1}, \\
\left|\varepsilon^{-3} \tau^{4}\right| \leqq b_{11}, \\
\left|\left(\varepsilon \tau^{-1}\right)^{-2}\left(\varepsilon \tau^{-2} x-1\right)-f_{+}\left(\varepsilon \tau^{-1}\right)\right| \leqq c_{2}, \\
\left|\varepsilon^{-3} \tau^{4}\left\{\left(\varepsilon \tau^{-1}\right)^{-2}\left(\varepsilon \tau^{-2} x-1\right)-f_{+}\left(\varepsilon \tau^{-1}\right)\right\}^{-\frac{3}{2}}\right| \leqq b_{14},
\end{array}\right.
$$

the system (W)' is transformed into a system of Type $\mathrm{C}$ if we apply a following transformation:

$$
\left\{\begin{array}{l}
\vec{y}=\left(\begin{array}{lc}
1 & 0 \\
0 & \varepsilon^{\frac{3}{2}} \tau^{-2} \cdot \varepsilon_{1} \cdot x_{4}^{\frac{1}{2}}
\end{array}\right) \overrightarrow{\mathfrak{w}}, \\
\varepsilon_{1}\left(=\varepsilon \tau^{-1}\right)=\beta, \\
\tau_{1}\left(\varepsilon_{1}^{-3} \tau=\varepsilon^{-3} \tau^{4}\right)=\alpha, \\
x_{4}\left(=x_{3}-f_{+}\left(\varepsilon_{1}\right)=\left(\varepsilon \tau^{-1}\right)^{-2}\left(\varepsilon \tau^{-2} x-1\right)-f_{+}\left(\varepsilon \tau^{-1}\right)\right)=\xi .
\end{array}\right.
$$

XLIV. In the subdomain

$$
\left\{\begin{array}{l}
\left|\varepsilon \tau^{-1}\right| \leqq a_{1}, \quad\left|\varepsilon \tau^{-2} x-1\right| \leqq c_{1}, \\
\left|\varepsilon^{-3} \tau^{4}\right| \leqq b_{11}, \\
\left|\left(\varepsilon \tau^{-1}\right)^{-2}\left(\varepsilon \tau^{-2} x-1\right)-f_{+}\left(\varepsilon \tau^{-1}\right)\right| \leqq c_{2}, \\
\left.\mid\left(\varepsilon^{-3} \tau^{4}\right)^{-\frac{2}{3}}\left\{\left(\varepsilon \tau^{-1}\right)^{-2}\left(\varepsilon \tau^{-2} x-1\right)-f_{+}\left(\varepsilon \tau^{-1}\right)\right\}\right) \leqq C_{15},
\end{array}\right.
$$

\section{a transformation}

$$
\left\{\begin{array}{l}
\vec{y}=\left(\begin{array}{cc}
1 & 0 \\
0 & \varepsilon^{\frac{3}{2}} \tau^{-2} \cdot \varepsilon_{1} \cdot \tau_{1}^{\frac{1}{3}}
\end{array}\right) \overrightarrow{\mathfrak{w}}, \\
\varepsilon_{1}\left(=\varepsilon \tau^{-1}\right)=\alpha, \\
\tau_{1}\left(=\varepsilon_{1}^{-3} \tau=\varepsilon^{-3} \tau^{4}\right)=\beta^{3}, \\
x_{5}\left(=\tau_{1}^{-\frac{2}{3}} x_{4}=\left(\varepsilon^{-3} \tau^{4}\right)^{-\frac{2}{3}}\left\{\left(\varepsilon \tau^{-1}\right)^{-2}\left(\varepsilon \tau^{-2} x-1\right)-f_{+}\left(\varepsilon \tau^{-1}\right)\right\}\right)=\xi,
\end{array}\right.
$$

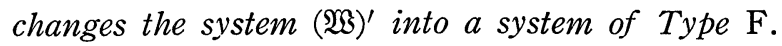


$\S \S 66$. Conclusion.

Summarizing the discussions done in this chapter, we can state the following :

Theorem 5. In order to construct an asymptotic expression of a fundamental set of independent solutions of the Whittaker's differential equation in the auxiliary subdomain

$$
c_{11} \leqq\left|\varepsilon \tau^{-2} x\right| \leqq C_{11}, \quad 0<|\varepsilon| \leqq a_{0}, \quad 0<|\tau| \leqq b_{0}, \quad 0<\mid x \leqq c_{0},
$$

it is sufficient to divide the domain $\left(\mathrm{D}_{11}\right)$ into following ten subdomains. The types of the reduced forms in each of such subdomains are given by a following table.

\begin{tabular}{l|c|c|c|c|c|c|c|c|c|c}
\hline Subdomain & $\mathrm{L}_{2}$ & $\mathrm{~L}_{3}$ & $\mathrm{~L}_{4}$ & $\mathfrak{\Omega}_{6}$ & $\mathfrak{\Omega}_{7}$ & $\mathfrak{\Omega}_{8}$ & $\mathrm{~L}_{10}$ & $\mathrm{~L}_{12}$ & $\mathfrak{\Omega}_{14}$ & $\mathfrak{\Omega}_{15}$ \\
\hline Normal form & $\mathrm{A}, \mathrm{C}, \mathrm{E}, \mathrm{F}$ & $\mathrm{G}$ & $\mathrm{E}$ & $\mathrm{A}$ & $\mathrm{E}$ & $\mathrm{F}$ & $\mathrm{F}$ & $\mathrm{E}$ & $\mathrm{C}$ & $\mathrm{F}$ \\
\hline Proposition & $\mathrm{XXXV}$ & $\mathrm{XXXVI}$ & $\mathrm{XXXVII}$ & $\mathrm{XXXVIII}$ & $\mathrm{XXXIX}$ & $\mathrm{XL}$ & $\mathrm{XLI}$ & $\mathrm{XLII}$ & $\mathrm{XLIII}$ & $\mathrm{XLIV}$ \\
\hline Section & 60 & 61 & 62 & 64 & 64 & 64 & 65 & 65 & 65 & 65 \\
\hline & $\mathrm{D}_{11}=\mathrm{L}_{1} \cup \mathrm{L}_{2} \cup \mathrm{L}_{3}$, \\
& $\mathrm{L}_{1}=\mathrm{L}_{4} \cup \mathrm{L}_{5}$, \\
& $\mathrm{L}_{5} \subset \mathfrak{\Omega}_{6} \cup \mathfrak{\Omega}_{7} \cup \mathfrak{\Omega}_{8} \cup \mathfrak{\Omega}_{9}$, \\
& $\mathfrak{L}_{9}=\mathrm{L}_{10} \cup \mathrm{L}_{11}$, \\
& $\mathrm{L}_{11}=\mathrm{L}_{12} \cup \mathrm{L}_{13}$, \\
& $\mathrm{L}_{13}=\mathfrak{\Omega}_{14} \cup \mathfrak{\Omega}_{15}$.
\end{tabular}

\section{Chapter IX. Asymptotic expression of fundamental solutions of the reduced forms of Types $A, B, C, D, E, F, G$.}

In the preceding chapters III VIII, we have discussed about a division of the domain

$$
(\varepsilon, \tau, x) \in\left\{\varepsilon:|\varepsilon| \leqq a_{0}\right\} \times\left\{\tau:|\tau| \leqq b_{0}\right\} \times\left\{x:|x| \leqq c_{0}\right\}
$$

into a finite number of subdomains in each of which the solution behaves quite differently. And, we have proved that in each of such subdomains the Whittaker's differential equation, after a suitable change of variables and parameters, is reduced to one of seven Types $\mathrm{A}(\S 12), \mathrm{B}(\S 15), \mathrm{C}(\S 19), \mathrm{D}(\S 18), \mathrm{E}(\S 20), \mathrm{F}(\S 22)$ and $\mathrm{G}(\S 34)$. Such types are called normal forms and have the following forms $\mathrm{s}^{5}$ :

$$
\begin{gathered}
a(\alpha, \beta, \xi) \cdot \xi \frac{d \overrightarrow{\mathfrak{w}}}{d \xi}=A(\alpha, \beta, \xi) \overrightarrow{\mathfrak{w}} \\
\left(\begin{array}{l}
\mathfrak{a}(\alpha, \beta, \xi) \equiv\left(\alpha^{l} \beta^{m} \xi^{n}\right)^{p}\left(\alpha^{l^{\prime}} \beta^{m \prime} \xi^{n^{\prime}}\right)^{p^{\prime}}\left(\alpha^{l^{\prime \prime}} \beta^{m \prime \prime} \xi^{n^{\prime \prime}}\right)^{p^{\prime \prime}} \\
p>0, \mathfrak{n} \mathfrak{p} \equiv n p+n^{\prime} p^{\prime}+n^{\prime \prime} p^{\prime \prime} \neq 0
\end{array}\right) ;
\end{gathered}
$$

5) The size of the matrices appearing in this chapter is 2 by 2 . 
(B)

$$
\begin{aligned}
& \mathfrak{a}(\alpha, \beta, \xi) \cdot \xi \frac{d \overrightarrow{\mathfrak{w}}}{d \xi}=A(\alpha, \beta, \xi) \overrightarrow{\mathfrak{w}} \\
& (p>0, \mathfrak{m p}=0) ; \\
& \left(\alpha \xi^{-\mu}\right)^{p}\left(\alpha^{-\nu} \xi\right)^{q} \cdot \xi \frac{d \overrightarrow{\mathfrak{w}}}{d \xi}=C(\alpha, \beta, \xi) \overrightarrow{\mathfrak{w}} \\
& (p>0,-\mu p+q \neq 0) ; \\
& \left(\alpha \xi^{-\mu}\right)^{p}\left(\alpha^{-\nu} \xi\right)^{q} \cdot \xi \frac{d \overrightarrow{\mathfrak{w}}}{d \xi}=C(\alpha, \beta, \xi) \overrightarrow{\mathfrak{w}} \\
& (p>0,-\mu p+q=0) ; \\
& \alpha^{p} \frac{d \overrightarrow{\mathfrak{w}}}{d \xi}=E(a, \beta, \xi) \overrightarrow{\mathfrak{w}} \quad(p>0) ; \\
& \frac{d \overrightarrow{\mathfrak{w}}}{d \xi}=F(\alpha, \beta, \xi) \overrightarrow{\mathfrak{w}} \\
& \alpha^{p} \beta^{q} \frac{d \overrightarrow{\mathfrak{w}}}{d \xi}=G(\alpha, \beta, \xi) \overrightarrow{\mathfrak{w}} \quad(p>0, q>0) .
\end{aligned}
$$

The purpose of the present chapter is to show how to construct an asymptotic expression of fundamental solutions.

The outline of our method is as follows:

We shall first remark that the coefficient matrix of the normal form, except for Type $F$, is supposed to have two distinct characteristic values in the considered domain. Next, using this property, we find a formal linear transformation which reduces formally the given system to two independent equations (which can be solved explicitly). Finally, we give an analytical meaning to such a formal transformation. Thus, we can obtain an asymptotic expression of fundamental solutions.

\section{§67. Type F.}

A system of Type $\mathrm{F}$ is written as

$$
\frac{d \overrightarrow{\mathfrak{w}}}{d \xi}=F(\alpha, \beta, \xi) \overrightarrow{\mathfrak{w}}
$$

The coefficient $F$ is supposed to be a matrix function holomorphic and bounded in $(\alpha, \beta, \xi)$ and admitting a uniform asymptotic expansion in powers of $\alpha$ :

$$
F(\alpha, \beta, \xi) \sim \sum_{h=0}^{\infty} \alpha^{h} F_{h}(\beta, \xi)
$$

as $\alpha$ tends to 0 in a domain

$$
\alpha \in \mathfrak{D}\left(\Theta_{ \pm} ; a\right), \quad \beta \in \mathcal{K}^{\prime}, \quad \xi \in \mathcal{K}^{\prime \prime},
$$

where $F_{h}(\beta, \xi)$ are matrix functions holomorphic and bounded in $(\beta, \xi) \in \mathcal{K}^{\prime} \times \mathcal{K}^{\prime \prime}$, $\mathcal{K}^{\prime}$ and $\mathcal{K}^{\prime \prime}$ being simply connected, open and bounded regions.

Such a system was studied by M. Hukuhara [2] in case when the given system ( $n$ equations) does not depend on the parameter $\beta$. By the same reasoning as in [2], we can easily prove the following: 
Lemma 1. The equation (F) admits a fundamental set of matrix solutions $W(\alpha, \beta, \xi)$, where $W(\alpha, \beta, \xi)$ is a non-singular matrix function with the same analytical significance as the matrix $F(\alpha, \beta, \xi)$.

\section{§68. Type E.}

A system of Type $\mathrm{E}$ is of the form

$$
\alpha^{p} \frac{d \overrightarrow{\mathfrak{w}}}{d \xi}=E(\alpha, \beta, \xi) \overrightarrow{\mathfrak{w}},
$$

where $E(\alpha, \beta, \xi)$ is supposed to be a matrix function holomorphic and bounded in $(\alpha, \beta, \xi)$ and uniformly asymptotically developable in powers of $\alpha$ :

$$
E(\alpha, \beta, \xi) \sim \sum_{h=0}^{\infty} \alpha^{h} E_{h}(\beta, \xi)
$$

as $\alpha$ tends to 0 in a domain

$$
\alpha \in \mathfrak{D}\left(\Theta_{ \pm} ; a\right), \quad \beta \in \mathcal{K}^{\prime}, \quad \xi \in \mathcal{K}^{\prime \prime},
$$

$\mathcal{K}^{\prime}$ and $\mathcal{K}^{\prime \prime}$ being simply connected, open and bounded regions, where $E_{h}(\beta, \xi)$ are matrix functions holomorphic and bounded in $(\beta, \xi) \in \mathcal{K}^{\prime} \times \mathcal{K}^{\prime \prime}$ and, especially, $E_{0}(\beta, \xi)$ has two distinct characteristic values there.

M. Hukuhara [2] showed a general method to construct an asymptotic expression of a system of fundamental solutions in case when the given system ( $n$ equations) does not contain the parameter $\beta$.

Without any essential modification of the method in [2], we can get an analogous result.

Let $\Theta_{0}, \Theta_{0}^{\prime}, \Theta_{0}^{\prime \prime}$ be arbitrarily given angles such that

$$
\left\{\begin{array}{l}
\Theta_{-}<\Theta_{0}<\Theta_{+}, \\
\mathcal{K}^{\prime} \cap\left\{\beta: \arg \beta=\Theta_{0}^{\prime}\right\} \neq \phi, \\
\mathcal{K}^{\prime \prime} \cap\left\{\xi: \arg \xi=\Theta_{0}^{\prime \prime}\right\} \neq \phi .
\end{array}\right.
$$

Put

$$
\left\{\begin{array}{l}
\widetilde{\Theta}_{ \pm}=\Theta_{0} \pm \gamma, \\
\widetilde{\mathcal{K}}^{\prime}=\mathcal{K}^{\prime} \cap \mathfrak{D}\left(\Theta_{0}^{\prime} \pm \gamma^{\prime} ; \infty\right), \\
\widetilde{\mathcal{K}}^{\prime \prime}=\mathcal{K}^{\prime \prime} \cap \mathfrak{D}\left(\Theta_{0}^{\prime \prime} \pm \gamma^{\prime \prime} ; \infty\right),
\end{array}\right.
$$

where $\gamma, \gamma^{\prime}, \gamma^{\prime \prime}$ are positive constants.

Let $P(\alpha, \beta, \xi)$ be a matrix which is nonsingular, holomorphic and bounded in $(\alpha, \beta, \xi)$ and admits a uniform asymptotic expansion:

$$
P(\alpha, \beta, \xi) \sim \sum_{h=0}^{\infty} \alpha^{h} P_{h}(\beta, \xi)
$$

as $\alpha$ tends to 0 in a domain

$$
\alpha \in \mathfrak{D}\left(\widetilde{\Theta}_{ \pm} ; a_{1}\right), \quad \beta \in \widetilde{\mathcal{K}}^{\prime}, \quad \xi \in \widetilde{\mathcal{K}}^{\prime \prime},
$$

where $P_{h}(\beta, \xi)$ are matrix functions holomorphic and bounded in $(\beta, \xi) \in \mathcal{K}^{\prime} \times \mathcal{K}^{\prime \prime}$.

Lemma 2. If we choose the positive constants $\gamma, \gamma^{\prime}, \gamma^{\prime \prime}$ and $a_{1}$ in a suitable way, there exists a transformation 


$$
\overrightarrow{\mathfrak{w}}=P(\alpha, \beta, \xi) \overrightarrow{\mathfrak{v}},
$$

where $P(\alpha, \beta, \xi)$ is a matrix satisfying the conditions given above, such that the system (E) is transformed into

$$
\alpha^{p} \frac{d \overrightarrow{\mathfrak{b}}}{d \xi}=\Lambda(\alpha, \beta, \xi) \overrightarrow{\mathfrak{v}},
$$

where $\Lambda(\alpha, \beta, \xi)$ is a diagonal matrix whose elements $\lambda_{i}(\alpha, \beta, \xi)(i=1,2)$ are polynomials of degree $p$ with respect to $\alpha$ and $\lambda_{i}(0, \beta, \xi)$ are the characteristic values of the matrix $E_{0}(\beta, \xi)$.

Remark. In case when $\mathcal{K}^{\prime}\left(\mathcal{K}^{\prime \prime}\right)$ contains the origin as an inner point, we can take $\tilde{\mathcal{K}}^{\prime}=\mathcal{K}^{\prime}\left(\tilde{\mathcal{K}}^{\prime \prime}=\mathcal{K}^{\prime \prime}\right)$.

§69. Type G.

A system of Type $\mathrm{G}$ will be written as

$$
\alpha^{p} \beta^{q} \frac{d \overrightarrow{\mathfrak{w}}}{d \xi}=G(\alpha, \beta, \xi) \overrightarrow{\mathfrak{w}} \quad(p>0, q>0) .
$$

The coefficient $G(\alpha, \beta, \xi)$ is supposed to be a matrix holomorphic and bounded in $(\alpha, \beta, \xi)$ and admitting a uniform asymptotic expansion in powers of $\alpha$ :

$$
G(\alpha, \beta, \xi) \sim \sum_{h=0}^{\infty} \alpha^{h} G_{h}(\beta, \xi)
$$

as $\alpha$ tends to 0 in a domain

$$
\alpha \in \mathfrak{D}\left(\Theta_{ \pm} ; a\right), \quad \beta \in \mathfrak{D}\left(\Theta_{ \pm}^{\prime} ; b\right), \quad \xi \in \mathcal{K}^{\prime \prime},
$$

$\mathcal{K}^{\prime \prime}$ being a simply connected, open and bounded region, where the coefficients $G_{h}(\beta, \xi)$ are matrix functions holomorphic and bounded in $(\beta, \xi)$ and asymptotically developable in powers of $\beta$ :

$$
G_{h}(\beta, \xi) \sim \sum_{k=0}^{\infty} \beta^{k} G_{h k}(\xi),
$$

uniformly valid for $\xi \in \mathcal{K}^{\prime \prime}$, as $\beta$ tends to 0 in the domain

$$
\beta \in \mathfrak{D}\left(\Theta_{ \pm}^{\prime} ; b\right), \quad \xi \in \mathcal{K}^{\prime \prime} .
$$

The coefficients $G_{h k}(\xi)$ are matrix functions holomorphic and bounded for $\xi \in \mathcal{K}^{\prime \prime}$ and, in particular, $G_{00}(\xi)$ has two distinct characteristic values there. We denote them by $\lambda_{i}(\xi)(i=1,2)$.

Such a system has been already studied by M. Iwano [3] (Theorem 10.1). His result can be stated as follows:

Let $\Theta_{0}, \Theta_{0}^{\prime}, \Theta_{0}^{\prime \prime}$ be arbitrarily given angles such that

$$
\left\{\begin{array}{l}
\Theta_{-}<\Theta_{0}<\Theta_{+}, \\
\Theta_{-}^{\prime}<\Theta_{0}^{\prime}<\Theta_{+}^{\prime}, \\
\mathcal{K}^{\prime \prime} \cap\left\{\xi: \arg \xi=\Theta_{0}^{\prime \prime}\right\} \neq \phi .
\end{array}\right.
$$

Put

$$
\left\{\begin{array}{l}
\widetilde{\Theta}_{ \pm}=\Theta_{0} \pm \gamma, \\
\widetilde{\Theta}_{ \pm}^{\prime}=\Theta_{0}^{\prime} \pm \gamma^{\prime}, \\
\widetilde{\mathcal{K}}^{\prime \prime}=\mathcal{K}^{\prime \prime} \cap \mathfrak{D}\left(\Theta_{0}^{\prime \prime} \pm \gamma^{\prime \prime} ; \infty\right),
\end{array}\right.
$$


where $\gamma, \gamma^{\prime}$ and $\gamma^{\prime \prime}$ are positive constants.

Let $P(\alpha, \beta, \xi)$ be a matrix function which is holomorphic, bounded in $(\alpha, \beta, \xi)$ and uniformly asymptotically developable in powers of $\alpha$ :

$$
P(\alpha, \beta, \xi) \sim \sum_{h=0}^{\infty} \alpha^{h} P_{h}(\beta, \xi)
$$

as $\alpha$ tends to 0 in a domain

$$
(\alpha, \beta, \xi) \in \mathfrak{D}\left(\widetilde{\Theta}_{ \pm} ; a_{1}\right) \times \mathfrak{D}\left(\widetilde{\Theta}_{ \pm}^{\prime} ; b_{1}\right) \times \widetilde{\mathcal{K}}^{\prime \prime}
$$

and, moreover, nonsingular there, where $P_{h}(\beta, \xi)$ are matrix functions with the same asymptotic properties as the matrix functions $G_{h}(\beta, \xi)$.

Lemma 3. For the suitably chosen positive constants $\gamma, \gamma^{\prime}, \gamma^{\prime \prime}, a_{1}, b_{1}$, there exists a nonsingular transformation

$$
\overrightarrow{\mathfrak{w}}=P(\alpha, \beta, \xi) \overrightarrow{\mathfrak{v}},
$$

where $P$ is a matrix satisfying the conditions given above, by which the system (G) is changed into a system

$$
\alpha^{p} \beta^{q} \frac{d \overrightarrow{\mathfrak{v}}}{d \xi}=\Lambda(\alpha, \beta, \xi) \overrightarrow{\mathfrak{b}} ;
$$

$\Lambda(\alpha, \beta, \xi)$ is a diagonal matrix whose elements $\lambda_{i}(\alpha, \beta, \xi)(i=1,2)$ are functions satisfying the same conditions as the matrix function $P(\alpha, \beta, \xi)$ and tend to $\lambda_{i}(\xi)$ as $\alpha, \beta$ tend to 0 in the domain (69.5).

Remark. From this lemma we see that the expressions $\lambda_{i}(\alpha, \beta, \xi)$ are power series of $\alpha$. However, if we restrict to a subdomain in $(\alpha, \beta)$-space such that $\left|\alpha \beta^{-\mu}\right| \leqq A$, where $\mu$ is a positive rational number, we can reduce $\lambda_{i}(\alpha, \beta, \xi)$ to a polynomial of degree $p_{0}$ with respect to $\alpha$ by applying a further transformation, $p_{0}$ being a positive integer satisfying the inequality $p_{0} \geqq p+q / \mu$. Concerning such a reduction, see also M. Iwano [3] (Theorem 11.2 in $\S 11$ ).

\section{$\S 70$. Type $C$ and Type $D$.}

We consider a system of the following form:

$$
\begin{gathered}
\left(\alpha \xi^{-\mu}\right)^{p}\left(\alpha^{-\nu} \xi\right)^{q} \cdot \xi \frac{d \overrightarrow{\mathfrak{w}}}{d \xi}=C(\alpha, \beta, \xi) \overrightarrow{\mathfrak{w}} \\
(\mu \geqq 0, \nu \geqq 0,0 \leqq \mu \nu<1, p>0, q \geqq 0,-\mu p+q \neq 0) .
\end{gathered}
$$

Here $C(\alpha, \beta, \xi)$ is supposed to be a matrix function holomorphic and bounded in $(\alpha, \beta, \xi)$ and uniformly asymptotically developable in powers of $\left(\alpha \xi^{-\mu}\right)$ :

$$
C(\alpha, \beta, \xi) \sim \sum_{h=0}^{\infty}\left(\alpha \xi^{-\mu}\right)^{h} C_{h}\left(\beta, \alpha^{-\nu \xi)}\right.
$$

as $\alpha$ tends to 0 in a domain

$$
\left\{\begin{array}{c}
\left|\alpha \xi^{-\mu}\right|<a^{\prime}, \quad\left|\alpha^{-\nu} \xi\right|<c^{\prime}, \\
\alpha \in \mathfrak{D}^{\prime}\left(\Theta_{ \pm} ; a\right), \quad \beta \in \mathcal{K}^{\prime}, \quad \xi \in \mathfrak{D}\left(\Theta_{ \pm}^{\prime \prime} ; c\right),
\end{array}\right.
$$

where $\mathcal{K}^{\prime}$ is a simply connected, open and bounded region. $C_{h}(\beta, \zeta)$ are matrix functions holomorphic and bounded in $(\beta, \zeta)$ and admitting uniform asymptotic expansions in powers of $\zeta$ : 


$$
C_{h}(\beta, \zeta) \sim \sum_{i=0}^{\infty} \zeta^{k} C_{h k}(\beta)
$$

as $\zeta$ tends to 0 in a domain

$$
\beta \in \mathcal{K}^{\prime}, \quad \zeta \in \mathfrak{D}\left(\Delta_{ \pm} ; d\right),
$$

where $C_{h k}(\beta)$ are matrix functions holomorphic and bounded for $\beta \in \mathcal{K}^{\prime}$ and, especially, $C_{00}(\beta)$ has two distinct characteristic values there.

In the case when the parameter $\beta$ is not contained, such a system has already appeared in the study of a system of linear ordinary differential equations containing a parameter as one of its reduced forms. M. Iwano [5] established a theorem concerning a so-called block-diagonalization, by which, in case the matrix $C_{00}(\beta)$ (independent of $\beta$ ) has all distinct characteristic values, we can construct an asymptotic expression of fundamental solutions.

However, in the present case, without any essential modification of the reasoning used in [5], we can obtain an analogous result.

We denote by $\lambda_{i}(\beta)(i=1,2)$ the characteristic values of the mataix $C_{00}(\beta)$. Let $\Theta_{0}, \Theta_{0}^{\prime}, \Theta_{0}^{\prime \prime}$ be arbitrarily given angles such that

$$
\left\{\begin{array}{l}
\Theta_{-}<\Theta_{0}<\Theta_{+}, \\
\mathcal{K}^{\prime} \cap\left\{\beta: \arg \beta=\Theta_{0}^{\prime}\right\} \neq \phi, \\
\Theta_{-}^{\prime \prime}<\Theta_{0}^{\prime \prime}<\Theta_{+}^{\prime \prime} .
\end{array}\right.
$$

An angular region

$$
\alpha \in \mathfrak{D}\left(\Theta_{0} \pm \gamma ; \infty\right), \quad \beta \in \mathfrak{D}\left(\Theta_{0}^{\prime} \pm \gamma^{\prime} ; \infty\right) \cap \mathcal{K}^{\prime}, \quad \xi \in \mathfrak{D}\left(\Theta_{0}^{\prime \prime} \pm \gamma^{\prime \prime} ; \infty\right)
$$

is said to be proper with respect to functions:

$$
\pm \int \frac{\lambda_{1}(\beta)-\lambda_{2}(\beta)}{\left(\alpha \xi^{-\mu}\right)^{p}\left(\alpha^{-\nu} \xi\right)^{q} \xi} d \xi \equiv \pm \frac{\lambda_{1}(\beta)-\lambda_{2}(\beta)}{(\mu p-q)\left(\alpha \xi^{-\mu}\right)^{p}\left(\alpha^{-\nu \xi}\right)^{q}}
$$

if the following inequality holds:

$$
\left|\arg \left\{ \pm \frac{\lambda_{1}(\beta)-\lambda_{2}(\beta)}{(\mu p-q)\left(\alpha \xi^{-\mu}\right)\left(\alpha^{-\nu} \xi\right)^{q}}\right\}\right| \leqq \frac{3}{2} \pi-\delta
$$

for (70.5), $\delta$ being a sufficiently small positive constant.

Since $\lambda_{1}(\beta)-\lambda_{2}(\beta)$ is supposed to be non-vanishing, it is easily proved that there exists such a proper region. For example, if $\gamma, \gamma^{\prime}, \gamma^{\prime \prime}$ are sufficiently small, the region (70.5) is certainly the one.

Now we suppose that the domain (70.5) is proper with respect to the functions (70.6).

Let $P(\alpha, \beta, \xi)$ be a matrix function which is nonsingular, holomorphic and bounded in $(\alpha, \beta, \xi)$ for

$$
\left\{\begin{array}{c}
\left|\alpha \xi^{-\mu}\right|<a_{1}^{\prime}, \quad \mid \alpha^{-\nu \xi \mid<c_{1}^{\prime},} \\
\alpha \in \mathfrak{D}\left(\widetilde{\Theta}_{ \pm} ; a_{1}\right), \quad \beta \in \widetilde{\mathcal{K}}^{\prime}, \quad \xi \in \mathfrak{D}\left(\widetilde{\Theta}_{ \pm}^{\prime \prime} ; c_{1}\right),
\end{array}\right.
$$

where

$$
\left\{\begin{array}{l}
\widetilde{\Theta}_{-}=\max \left(\Theta_{-}, \Theta_{0}-\gamma\right), \quad \widetilde{\Theta}_{+}=\min \left(\Theta_{+}, \Theta_{0}+\gamma\right) ; \\
\widetilde{\mathcal{K}}^{\prime}=\mathcal{K}^{\prime} \cap \mathscr{D}\left(\Theta_{0}^{\prime} \pm \gamma^{\prime} ; \infty\right) ; \\
\widetilde{\Theta}_{-}^{\prime \prime}=\max \left(\Theta_{-}^{\prime \prime}, \Theta_{0}^{\prime \prime}-\gamma^{\prime \prime}\right), \quad \widetilde{\Theta}_{+}^{\prime \prime}=\min \left(\Theta_{+}^{\prime \prime}, \Theta_{0}^{\prime \prime}+\gamma^{\prime \prime}\right) .
\end{array}\right.
$$


Moreover $P(\alpha, \beta, \xi)$ admits a uniform asymptotic expansion

$$
P(\alpha, \beta, \xi) \sim \sum_{h=0}^{\infty}\left(\alpha \xi^{-\mu}\right)^{h} P_{h}\left(\beta, \alpha^{-\nu} \xi\right)
$$

as $\alpha$ tends to 0 in the domain (70.8), where $P_{h}(\beta, \zeta)$ are matrix functions with the same analytical meaning as the matrices $C_{h}(\beta, \zeta)$.

Lemma 4. If we choose the positive constants $\gamma, \gamma^{\prime}, \gamma^{\prime \prime}, a_{1}, c_{1}, a_{1}^{\prime}, c_{1}^{\prime}$ in a suitable way, there exists a nonsingular transformation

$$
\overrightarrow{\mathfrak{w}}=P(\alpha, \beta, \xi) \overrightarrow{\mathfrak{p}},
$$

where $P$ is a matrix function satisfying the conditions given above, which transforms the system (C) into

$$
\left(\alpha \xi^{-\mu}\right)^{p}\left(\alpha^{-\nu \xi}\right)^{q} \cdot \xi \frac{\overrightarrow{d \mathfrak{b}}}{d \xi}=\Lambda(\alpha, \beta, \xi) \overrightarrow{\mathfrak{b}},
$$

where $\Lambda(\alpha, \beta, \xi)$ is a diagonal matrix whose elements $\lambda_{i}(\alpha, \beta, \xi)(i=1,2)$ are functions with the same properties as the matrix $P(\alpha, \beta, \xi)$ and tend to $\lambda_{i}(\beta)$ as $\left(\alpha \xi^{-\mu}\right)$ and $\left(\alpha^{-\nu} \xi\right)$ tend to 0 .

Next we will study a system of Type D:

$$
\left(\alpha \xi^{-\mu}\right)^{p}\left(\alpha^{-\nu \xi}\right)^{q} \cdot \xi \frac{d \overrightarrow{\mathfrak{w}}}{d \xi}=C(\alpha, \beta, \xi) \overrightarrow{\mathfrak{w}} .
$$

This equation satisfies the same conditions as those imposed upon Type $\mathrm{C}$ except for the condition $-\mu p+q=0$. Hence, in this case, the expression $\left(\alpha \xi^{-\mu}\right)^{p}\left(\alpha^{-\nu \xi}\right)^{q}$ does not depend on the independent variable $\xi$. Therefore, the functions (70.6) will be reduced to

$$
\pm \int \frac{\lambda_{1}(\beta)-\lambda_{2}(\beta)}{\left(\alpha \xi^{-\mu}\right)^{p}\left(\alpha^{-\nu} \xi\right)^{q} \xi} d \xi \equiv \pm \frac{\lambda_{1}(\beta)-\lambda_{2}(\beta)}{\left(\alpha \xi^{-\mu}\right)^{p}\left(\alpha^{-\nu \xi}\right)^{q}} \log \xi
$$

A system $\left(\theta^{*}, \theta^{*}\right)$ of angles $\theta^{*}$ and $\theta^{\prime *}$ such that

$$
\left|\arg \left\{ \pm \frac{\lambda_{1}(\beta)-\lambda_{2}(\beta)}{\left(\alpha \xi^{-\mu}\right)^{p}\left(\alpha^{-\nu} \xi\right)^{q}}\right\}\right|=\frac{\pi}{2}
$$

for $\arg \alpha=\theta^{*}$ and $\arg \beta=\theta^{\prime *}$, is called singular with respect to the functions $(70.6)^{\prime}$.

Let $\Theta_{0}, \Theta_{0}^{\prime}, \gamma, \gamma^{\prime}$ be the same as before. Then, a domain

$$
\alpha \in \mathfrak{D}\left(\Theta_{0} \pm \gamma ; \infty\right), \quad \beta \in \mathfrak{D}\left(\Theta_{0}^{\prime} \pm \gamma^{\prime} ; \infty\right) \cap \mathcal{K}^{\prime}
$$

is said to be proper with respect to the functions $(70.6)^{\prime}$ when any system $\left(\theta, \theta^{\prime}\right)$ of angles $\theta$ and $\theta^{\prime}$ such that

$$
\Theta_{0}-r \leqq \theta \leqq \Theta_{0}+r, \quad \Theta_{0}^{\prime}-\gamma^{\prime} \leqq \theta^{\prime} \leqq \Theta_{0}^{\prime}+\gamma^{\prime}
$$

is not singular.

Suppose that the domain (70.12) is proper with respect to the functions $(70.6)^{\prime}$. Put

$$
\left\{\begin{array}{l}
\widetilde{\Theta}_{-}=\max \left(\Theta_{-}, \Theta_{0}-\gamma\right), \quad \widetilde{\Theta}_{+}=\min \left(\Theta_{+}, \Theta_{0}+\gamma\right) ; \\
\widetilde{\mathcal{K}}^{\prime}=\mathcal{K}^{\prime} \cap \mathfrak{D}\left(\Theta_{0}^{\prime} \pm \gamma^{\prime} ; \infty\right) .
\end{array}\right.
$$

Let $P(\alpha, \beta, \xi)$ be a nonsingular matrix function which is holomorphic and bounded in $(\alpha, \beta, \xi)$ for 


$$
\left\{\begin{array}{c}
\left|\alpha \xi^{-\mu}\right|<a_{1}^{\prime}, \quad \mid \alpha^{-\nu \xi \mid<c_{1}^{\prime},} \\
\alpha \in \mathscr{D}\left(\widetilde{\Theta}_{ \pm} ; a_{1}\right), \quad \beta \in \widetilde{\mathcal{K}}^{\prime}, \quad \xi \in \mathfrak{D}\left(\Theta_{ \pm}^{\prime \prime} ; c_{1}\right)
\end{array}\right.
$$

and, moreover, possesses the asymptotic behavior similar to the matrix $P$ appeared in the discussion of Type $\mathrm{C}$, where $\Theta_{ \pm}^{\prime \prime}$ coincide with those appearing in (70.2).

Lemma 5. If we choose the positive quantities $a_{1}, c_{1}, \gamma, \gamma^{\prime}, a_{1}^{\prime}, c_{1}^{\prime}$ in a suitable way, there exists a transformation of the form (70.10) by which the transformed system has the same form as (70.11).

Remarks. 1. If we restrict to a subdomain of (70.8) such that $\left|\alpha \xi^{-\mu^{\prime}}\right|<A$, where $\mu^{\prime}$ is an arbitrarily given rational number satisfying $\mu<\mu^{\prime}<1 / \nu$, we can reduce the expressions $\lambda_{i}(\alpha, \beta, \xi)$ to polynomials of $\left(\alpha \xi^{-\mu^{\prime}}\right)$ by applying a further transformation.

2. In Type $\mathrm{C}$ we have supposed $p>0$. In case when $p=0$ and $q>0$, let (70.5) be an arbitrary proper region with respect to the functions (70.6) (where we have to put $p=0$ ). Then, the lemma 4 still holds if we replace the domain (70.4), where the matrix functions $P_{h}(\beta, \zeta)$ appearing in the asymptotic series (70.9) are defined, by a following domain

where

$$
\beta \in \widetilde{\mathcal{K}}^{\prime}, \quad \zeta \in \mathbb{D}\left(\widetilde{\Delta}_{ \pm} ; d\right),
$$

$$
\left\{\begin{array}{l}
\widetilde{\mathcal{K}}^{\prime}=\mathcal{K}^{\prime} \cap \mathfrak{D}\left(\Theta_{0}^{\prime} \pm \gamma^{\prime} ; \infty\right) ; \\
\widetilde{\Delta}_{-}=\max \left(\Delta_{-}, \Theta_{0}^{\prime \prime}-\gamma^{\prime \prime}-\nu\left(\Theta_{0}+\gamma\right)\right), \\
\widetilde{\Delta}_{+}=\min \left(\Delta_{+}, \Theta_{0}^{\prime \prime}+\gamma^{\prime \prime}-\nu\left(\Theta_{0}-\gamma\right)\right) .
\end{array}\right.
$$

\section{§71. Type A and Type B.}

A system of Type A will be given in a form:

$$
\alpha(\alpha, \beta, \xi) \cdot \xi \frac{d \overrightarrow{\mathfrak{w}}}{d \xi}=A(\alpha, \beta, \xi) \overrightarrow{\mathfrak{w}}
$$

Here $A(\alpha, \beta, \xi)$ is supposed to be a matrix function holomorphic and bounded in $(\alpha, \beta, \xi)$ and, moreover, uniformly asymptotically developable in a form

$$
A(\alpha, \beta, \xi) \sim \sum_{h=0}^{\infty}\left(\alpha^{l} \beta^{m} \xi^{n}\right)^{h} A_{h}\left(\alpha^{l^{\prime}} \beta^{m^{\prime}} \xi^{n^{\prime}}, \alpha^{l^{\prime \prime}} \beta^{m^{\prime \prime}} \xi^{n^{\prime \prime}}\right)
$$

as $\alpha$ and $\beta$ tend to 0 in a domain

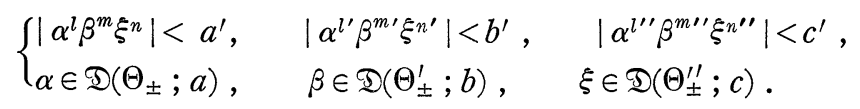

$A_{h}(\eta, \zeta)$ are matrix functions holomorphic and bounded in $(\eta, \zeta)$ and admitting uniform asymptotic expansions:

$$
A_{h}(\eta, \zeta) \sim \sum_{k=0}^{\infty} \eta^{k} A_{h k}(\zeta)
$$

as $\eta$ tends to 0 in a domain

$$
\eta \in \mathfrak{D}\left(\Delta_{ \pm}^{\prime} ; d^{\prime}\right), \quad \zeta \in \mathfrak{D}\left(\Delta_{ \pm}^{\prime \prime} ; d^{\prime \prime}\right),
$$

where $A_{h k}(\zeta)$ are matrix functions holomorphic, bounded and asymptotically 
developable in powers of $\zeta$ :

$$
A_{h k}(\zeta) \sim \sum_{j=0}^{\infty} \zeta^{j} A_{h k j}
$$

as $\zeta$ tends to 0 in the domain

$$
\zeta \in \mathfrak{D}\left(\Delta_{ \pm}^{\prime \prime} ; d^{\prime \prime}\right)
$$

$A_{h k j}$ being constant matrices. Further, the matrix $A_{000}$ has two distinct characteristic values.

We denote by $\lambda_{i}(j=1,2)$ the characteristic values of the matrix $A_{000}$. Let

$$
\arg \alpha=\Theta_{0}, \quad \arg \beta=\Theta_{0}^{\prime}, \quad \arg \xi=\Theta_{0}^{\prime \prime}
$$

be arbitrarily given directions contained in (71.2). A domain

$$
\alpha \in \mathfrak{D}\left(\Theta_{0} \pm \gamma ; \infty\right), \quad \beta \in \mathfrak{D}\left(\Theta_{0}^{\prime} \pm \gamma^{\prime} ; \infty\right), \quad \xi \in \mathfrak{D}\left(\Theta_{0}^{\prime \prime} \pm \gamma^{\prime \prime} ; \infty\right)
$$

is said to be proper with respect to functions

$$
\pm \int \frac{\lambda_{1}-\lambda_{2}}{\mathfrak{a}(\alpha, \beta, \xi) \xi} d \xi=\mp \frac{\lambda_{1}-\lambda_{2}}{\mathfrak{m p} \cdot \mathfrak{a}(\alpha, \beta, \xi)}
$$

if we have the inequality

$$
\left|\arg \left\{ \pm \frac{\lambda_{1}-\lambda_{2}}{n \mathfrak{p} \cdot \mathfrak{a}(\alpha, \beta, \xi)}\right\}\right| \leqq \frac{3 \pi}{2}-\delta
$$

for (71.7), where $\delta$ is a sufficiently small positive quantity.

Since $\lambda_{1}-\lambda_{2}$ is supposed to be different from zero, we can easily prove the existence of such a proper region. For example, if $\gamma, \gamma^{\prime}$ and $\gamma^{\prime \prime}$ are sufficiently small, the region (71.7) is certainly the one.

Now we suppose that the region (71.7) is proper. Let $P(\alpha, \beta, \xi)$ be a matrix function which is nonsingular, holomorphic and bounded in $(\alpha, \beta, \xi)$ for



where

$$
\begin{cases}\tilde{\Theta}_{+}=\min \left(\Theta_{+}, \Theta_{0}+\gamma\right), & \tilde{\Theta}_{-}=\max \left(\Theta_{-}, \Theta_{0}-\gamma\right) ; \\ \tilde{\Theta}_{+}^{\prime}=\min \left(\Theta_{+}^{\prime}, \Theta_{0}^{\prime}+\gamma^{\prime}\right), & \tilde{\Theta}_{-}^{\prime}=\max \left(\Theta_{-}^{\prime}, \Theta_{0}^{\prime}-\gamma^{\prime}\right) ; \\ \tilde{\Theta}_{+}^{\prime \prime}=\min \left(\Theta_{+}^{\prime \prime}, \Theta_{0}^{\prime \prime}+\gamma^{\prime \prime}\right), & \tilde{\Theta}_{-}^{\prime \prime}=\max \left(\Theta_{-}^{\prime \prime}, \Theta_{0}^{\prime \prime}-\gamma^{\prime \prime}\right) .\end{cases}
$$

Moreover, $P(\alpha, \beta, \xi)$ admits a uniform asymptotic expansion:

$$
P(\alpha, \beta, \xi) \sim \sum_{h=0}^{\infty}\left(\alpha^{l} \beta^{m} \xi^{n}\right)^{h} P_{h}\left(\alpha^{l^{\prime}} \beta^{m^{\prime}} \xi^{n^{\prime}}, \alpha^{l^{\prime}} \beta^{m^{\prime \prime} \xi^{n^{\prime \prime}}}\right)
$$

as $\alpha$ and $\beta$ tend to 0 in the domain (71.10), where the coefficients $P_{h}(\eta, \zeta)$ are matrix functions satisfying the same conditions as the matrices $A_{h}(h, \zeta)$ appearing in (71.1).

Lemma 6. For the suitably chosen positive constants $\gamma, \gamma^{\prime}, \gamma^{\prime}, a_{1}, b_{1}, c_{1}, a_{1}^{\prime}, b_{1}^{\prime}, c_{1}^{\prime}$, there exists a transformation

$$
\overrightarrow{\mathfrak{w}}=P(\alpha, \beta, \xi) \overrightarrow{\mathfrak{v}}
$$


where $P(\alpha, \beta, \xi)$ is a matrix function satisfying the conditions given above, such that the system (A) is transformed into a system

$$
\mathfrak{a}(\alpha, \beta, \xi) \cdot \xi \frac{d \overrightarrow{\mathfrak{b}}}{d \xi}=\Lambda(\alpha, \beta, \xi) \overrightarrow{\mathfrak{b}},
$$

where $\Lambda(\alpha, \beta, \xi)$ is a diagonal matrix whose elements $\lambda_{i}(\alpha, \beta, \xi)(i=1,2)$ are functions with the same properties as the matrix $P(\alpha, \beta, \xi)$ and tend to $\lambda_{i}$ as $\left|\alpha^{l} \beta^{m} \xi^{n}\right|$, $\left|\alpha^{l^{\prime}} \beta^{m^{\prime}} \xi^{n^{\prime}}\right|,\left|\alpha^{l^{\prime \prime}} \beta^{m^{\prime \prime}} \xi^{n^{\prime \prime}}\right|$ tend to 0 .

Next, we consider a system of Type B which has a form similar to Type A:

$$
a(\alpha, \beta, \xi) \cdot \xi \frac{d \overrightarrow{\mathfrak{w}}}{d \xi}=A(\alpha, \beta, \xi) \overrightarrow{\mathfrak{w}},
$$

where $A(\alpha, \beta, \xi)$ is a matrix function satisfying the same conditions as before. The only distinction between Type $A$ and Type B consists in that $n \mathfrak{p}=0$ and, consequently, the function $\mathfrak{a}(\alpha, \beta, \xi)$ does not depend on the independent variable $\xi$. Therefore, in this case, the functions (71.8) will be reduced to

$$
\pm \int \frac{\lambda_{1}-\lambda_{2}}{\mathfrak{a}(\alpha, \beta, \xi) \cdot \xi} d \xi= \pm \frac{\lambda_{1}-\lambda_{2}}{\mathfrak{a}(\alpha, \beta, \xi)} \log \xi \text {. }
$$

A system $\left(\theta^{*}, \theta^{\prime *}\right)$ of angles $\theta^{*}$ and $\theta^{*}$ such that

$$
\left|\arg \left\{ \pm \frac{\lambda_{1}-\lambda_{2}}{\mathfrak{a}(\alpha, \beta, \xi)}\right\}\right|=\frac{\pi}{2}
$$

for $\arg \alpha=\theta^{*}$ and $\arg \beta=\theta^{\prime *}$, is called singular with respect to the functions $(71.8)^{\prime}$.

Let $\Theta_{0}, \Theta_{0}^{\prime}, \gamma, \gamma^{\prime}$ be the same as before. A domain

$$
\alpha \in \mathfrak{D}\left(\Theta_{0} \pm \gamma ; \infty\right), \quad \beta \in \mathfrak{D}\left(\Theta_{0}^{\prime} \pm \gamma^{\prime} ; \infty\right)
$$

is said to be proper with respect to the functions $(71.8)^{\prime}$ when any system $\left(\theta, \theta^{\prime}\right)$ of angles $\theta$ and $\theta^{\prime}$, such that

is not singular.

$$
\Theta_{0}-\gamma \leqq \theta \leqq \Theta_{0}+\gamma, \quad \Theta_{0}^{\prime}-\gamma^{\prime} \leqq \theta^{\prime} \leqq \Theta_{0}^{\prime}+\gamma^{\prime}
$$

Assume the domain (71.14) is proper with respect to the function $(71.8)^{\prime}$.

Let $P(\alpha, \beta, \xi)$ be a nonsingular matrix function which is holomorphic and bounded in $(\alpha, \beta, \xi)$ for

$$
\left\{\begin{array}{lll}
\left|\alpha^{l} \beta^{m} \xi^{n}\right|<a_{1}^{\prime}, & \left|\alpha^{l^{\prime}} \beta^{m^{\prime}} \xi^{n^{\prime}}\right|<b_{1}^{\prime}, & \left|\alpha^{l^{\prime \prime}} \beta^{m^{\prime \prime}} \xi^{n^{\prime \prime}}\right|<c_{1}^{\prime}, \\
\alpha \in \mathfrak{D}\left(\widetilde{\Theta}_{ \pm} ; a_{1}\right), \quad \beta \in \mathfrak{D}\left(\widetilde{\Theta}_{ \pm}^{\prime} ; b_{1}\right), & \xi \in \mathscr{D}\left(\Theta_{ \pm}^{\prime \prime} ; c_{1}\right),
\end{array}\right.
$$

where

$$
\begin{cases}\widetilde{\Theta}_{+}=\min \left(\Theta_{+}, \Theta_{0}+\gamma\right), & \widetilde{\Theta}_{-}=\max \left(\Theta_{-}, \Theta_{0}-\gamma\right) ; \\ \widetilde{\Theta}_{+}^{\prime}=\min \left(\Theta_{+}^{\prime}, \Theta_{0}^{\prime}+\gamma^{\prime}\right), & \widetilde{\Theta}_{-}^{\prime}=\max \left(\Theta_{-}^{\prime}, \Theta_{0}^{\prime}-\gamma^{\prime}\right) .\end{cases}
$$

Further, $P$ admits the asymptotic behavior similar to the matrix $P(\alpha, \beta, \xi)$ appeared in the discussion of Type A.

Lemma 7. If we choose the positive quantities $a_{1}, b_{1}, c_{1}, \gamma, \gamma^{\prime}, a_{1}^{\prime}, b_{1}^{\prime}, c_{1}^{\prime}$ in a suitable way, there exists a transformation of the form (71.12) by which the transformed system has the same form as (71.13).

Remark. In a certain case (for example, see the system (15.2) in Chapter 
III), $a(\alpha, \beta, \xi)$ may depend on only one parameter, say $\alpha$. In this case, for a particular angle $\theta^{*}$ and for an arbitrary angle $\theta^{\prime *}$, a system $\left(\theta^{*}, \theta^{*}\right)$ may be singular. The angles $\Theta_{ \pm}^{\prime \prime}$ appearing in (71.15) coincide with those appearing in (71.2).

\section{$\S 72$. Outline of the proof of Lemmas 6 and 7 .}

We consider a system of Type A or Type B :

$$
\mathfrak{a}(\alpha, \beta, \xi) \cdot \xi \frac{d \overrightarrow{\mathfrak{w}}}{d \xi}=A(\alpha, \beta, \xi) \overrightarrow{\mathfrak{w}},
$$

where

$$
\begin{array}{r}
\mathfrak{a}(\alpha, \beta, \xi) \equiv\left(\alpha^{l} \beta^{m} \xi^{n}\right)^{p}\left(\alpha^{l^{\prime}} \beta^{m^{\prime}} \xi^{n^{\prime}}\right)^{p^{\prime}}\left(\alpha^{l^{\prime \prime}} \beta^{m^{\prime \prime}} \xi^{n^{\prime \prime}}\right)^{p^{\prime \prime}}, \\
p>0, \quad \mathfrak{n} \mathfrak{p} \equiv n p+n^{\prime} p^{\prime}+n^{\prime \prime} p^{\prime \prime} \begin{cases}\neq 0 & (\text { Type A) }, \\
=0 & \text { (Type B) } .\end{cases}
\end{array}
$$

Here $A(\alpha, \beta, \xi)$ admits a uniform asymptotic expansion

$$
A(\alpha, \beta, \xi) \sim \sum_{h=0}^{\infty}\left(\alpha^{l} \beta^{m} \xi^{n}\right)^{h} A_{h}\left(\alpha^{l^{\prime}} \beta^{m^{\prime}} \xi^{n^{\prime}}, \alpha^{l^{\prime \prime}} \beta^{m^{\prime \prime}} \xi^{n^{\prime \prime}}\right)
$$

as $\alpha$ and $\beta$ tend to 0 in a domain

$$
\left\{\begin{array}{lll}
\left|\alpha^{l} \beta^{m} \xi^{n}\right|<a^{\prime}, & \left|\alpha^{l^{\prime}} \beta^{m^{\prime}} \xi^{n^{\prime}}\right|<b^{\prime}, & \left|\alpha^{l^{\prime \prime}} \beta^{m^{\prime \prime}} \xi^{n^{\prime \prime}}\right|<c^{\prime}, \\
\alpha \in \mathscr{D}\left(\Theta_{ \pm} ; a\right), & \beta \in \mathscr{D}\left(\Theta_{ \pm}^{\prime} ; b\right), & \xi \in \mathscr{D}\left(\Theta_{ \pm}^{\prime \prime} ; c\right),
\end{array}\right.
$$

where the coefficients $A_{h}(\eta, \zeta)$ admit uniform asymptotic expansions

$$
A_{h}(\eta, \zeta) \sim \sum_{k=0}^{\infty} \eta^{k} A_{h k}(\zeta)
$$

as $\eta$ tends to 0 in a domain

$$
\eta \in \mathscr{D}\left(\Delta_{ \pm}^{\prime} ; d^{\prime}\right), \quad \zeta \in \mathfrak{D}\left(\Delta_{ \pm}^{\prime \prime} ; d^{\prime \prime}\right) .
$$

Further, $A_{k k}(\zeta)$ are asymptotically developable in powers of $\zeta$ for the domain

$$
\zeta \in \mathfrak{D}\left(\Delta_{ \pm}^{\prime \prime} ; d^{\prime \prime}\right)
$$

\section{$1^{\circ}$. Preliminary reduction.}

We will first show that:

We can suppose without loss of generality that the matrix $A_{0}(\eta, \zeta)$ is a diagonal matrix whose elements are $\lambda_{i}(\eta, \zeta)(i=1,2)$.

Indeed, since the matrix $A_{000}$ defined by $\lim _{\zeta \rightarrow 0} A_{00}(\zeta)$ is supposed to have two distinct characteristic values, say $\lambda_{i}(i=1,2)$, we can suppose that the matrix $A_{0}(\eta, \zeta)$ has two distinct characteristic values $\lambda_{i}(\eta, \zeta)$ in the domain (72.6) such that $\lim _{\eta \rightarrow 0} \lambda_{i}(\eta, \zeta)=\lambda_{i} \quad(i=1,2)$. Then, it is well known that there exists a nonsingular matrix function $P_{0}(\eta, \zeta)$ with the same asymptotic property as that of the matrix $A_{0}(\eta, \zeta)$ such that $P_{0}(\eta, \zeta)^{-1} A_{0}(\eta, \zeta) P_{0}(\eta, \zeta)$ is reduced to a diagonal matrix with diagonal elements $\lambda_{i}(\eta, \zeta)(i=1,2)$ in the domain (72.6).

Now let us make a transformation

$$
\overrightarrow{\mathfrak{w}}=P_{0}\left(\alpha^{l^{\prime}} \beta^{m^{\prime}} \xi^{n^{\prime}}, \alpha^{l^{\prime \prime}} \beta^{m^{\prime \prime}} \xi^{n^{\prime \prime}}\right) \overrightarrow{\mathfrak{w}}_{1}
$$

Then the transformed system is easily found to be 


$$
\mathfrak{a}(\alpha, \beta, \xi) \cdot \xi \frac{d \overrightarrow{\mathfrak{w}}_{1}}{d \xi}=B(\alpha, \beta, \xi) \overrightarrow{\mathfrak{w}}_{1},
$$

where $B(\alpha, \beta, \xi)$ admits the uniform asymptotic expansion

$$
B(\alpha, \beta, \xi) \sim \sum_{h=0}^{\infty}\left(\alpha^{l} \beta^{m} \xi^{n}\right)^{h} B_{h}\left(\alpha^{l^{\prime}} \beta^{m^{\prime}} \xi^{n^{\prime}}, \alpha^{l^{\prime \prime}} \beta^{m^{\prime \prime}} \xi^{n^{\prime \prime}}\right)
$$

in the domain (72.4). Clearly,

$$
B_{h}(\eta, \zeta)=\left\{\begin{array}{l}
P_{0}(\eta, \zeta)^{-1} A_{h}(\eta, \zeta) P_{0}(\eta, \zeta) \quad(h \neq p), \\
P^{-1} A_{h} P_{0}-\eta^{p^{\prime}} \zeta^{p^{\prime \prime}} P^{-1}\left\{n^{\prime} \eta \frac{\partial P_{0}}{\partial \eta}+n^{\prime \prime} \zeta \frac{\partial P_{0}}{\partial \zeta}\right\} \quad(h=p) .
\end{array}\right.
$$

Consequently, since $p>0, B_{0}(\eta, \zeta)$ becomes a diagonal matrix.

Therefore, we suppose at the outset that the matrix $A_{0}(\eta, \zeta)$ has a diagonal form.

\section{$2^{\circ}$. Diagonalizing transformation.}

Let us consider a formal transformation

$$
\overrightarrow{\mathfrak{w}}=Q(\alpha, \beta, \xi) \overrightarrow{\mathfrak{v}},
$$

where $Q(\alpha, \beta, \xi)$ is a formal matrix function which is formally developable in powers of $\left(\alpha^{l} \beta^{m} \xi^{n}\right)$. If we write the transformed system as

$$
\mathfrak{a}(\alpha, \beta, \xi) \cdot \xi \frac{d \overrightarrow{\mathfrak{v}}}{d \xi}=\Lambda(\alpha, \beta, \xi) \overrightarrow{\mathfrak{v}},
$$

we see by a simple calculation that the following relation must hold :

$$
a(\alpha, \beta, \xi) \cdot \xi \frac{d Q}{d \xi}=A(\alpha, \beta, \xi) Q-Q \Lambda(\alpha, \beta, \xi) .
$$

We put here

$$
\begin{aligned}
& A(\alpha, \beta, \xi)=\left(\begin{array}{ccc}
\lambda_{1}\left(\alpha^{l^{\prime}} \beta^{m^{\prime}} \xi^{n^{\prime}}, \alpha^{l^{\prime \prime}} \beta^{m^{\prime \prime}} \xi^{n^{\prime \prime}}\right)+B_{11}(\alpha, \beta, \xi) & B_{12}(\alpha, \beta, \xi) \\
B_{21}(\alpha, \beta, \xi) & \lambda_{2}\left(\alpha^{l^{\prime}} \beta^{m^{\prime}} \xi^{n^{\prime}}, \alpha^{l^{\prime \prime}} \beta^{m^{\prime \prime}} \xi^{n^{\prime \prime}}\right)+B_{22}(\alpha, \beta, \xi)
\end{array}\right), \\
& \Lambda(\alpha, \beta, \xi)=\left(\begin{array}{ccc}
\lambda_{1}\left(\alpha^{l^{\prime}} \beta^{m^{\prime}} \xi^{n^{\prime}}, \alpha^{l^{\prime \prime}} \beta^{m^{\prime \prime}} \xi^{n^{\prime \prime}}\right)+\Lambda_{1}(\alpha, \beta, \xi) & 0 \\
0 & \lambda_{2}\left(\alpha^{l^{\prime}} \beta^{m^{\prime}} \xi^{n^{\prime}}, \alpha^{l^{\prime \prime}} \beta^{m^{\prime \prime}} \xi^{n^{\prime \prime}}\right)+\Lambda_{2}(\alpha, \beta, \xi)
\end{array}\right), \\
& Q(\alpha, \beta, \xi)=\left(\begin{array}{cc}
1 & Q_{12}(\alpha, \beta, \xi) \\
Q_{21}(\alpha, \beta, \xi) & 1
\end{array}\right),
\end{aligned}
$$

where $B_{i j}, \Lambda_{i}$ and $Q_{i j}$ are functions which are formally developable in power series of $\left(\alpha^{l} \beta^{m} \xi^{n}\right)$. Of course, $B_{i j}(\alpha, \beta, \xi)$ are known functions.

Then, we can easily see that the matrix equation (72.10) is equivalent to the following four equations :

$$
\begin{cases}0=B_{12} Q_{21}+B_{11}-\Lambda_{1}, & (1,1) \text {-element } \\ 0=B_{21} Q_{12}+B_{22}-\Lambda_{2}, & (2,2) \text {-element } \\ \mathfrak{a} \cdot \xi \frac{d Q_{12}}{d \xi}=\left(\lambda_{1}-\lambda_{2}\right) Q_{12}+B_{12}+\left(B_{11}-\Lambda_{2}\right) Q_{12}, & (1,2) \text {-element } \\ \mathfrak{a} \cdot \xi \frac{d Q_{21}}{d \xi}=\left(\lambda_{2}-\lambda_{1}\right) Q_{21}+B_{21}+\left(B_{22}-\Lambda_{1}\right) Q_{21}, & (2,1) \text {-element }\end{cases}
$$


where the notation $\lambda_{i}=\lambda_{i}\left(\alpha^{l^{\prime}} \beta^{m^{\prime}} \xi^{n^{\prime}}, \alpha^{l^{\prime \prime}} \beta^{m^{\prime \prime}} \xi^{n^{\prime \prime}}\right)$ is adopted for simplicity's sake.

By solving from the first two equations the expressions $\Lambda_{1}(\alpha, \beta, \xi)$ and $\Lambda_{2}(\alpha, \beta, \xi)$, and by substituting them into the last two equations, we obtain the following two independent nonlinear differential equations :

$$
\begin{aligned}
a(\alpha, \beta, \xi) \cdot \xi \frac{d Q_{12}}{d \xi}= & \left(\lambda_{1}-\lambda_{2}\right) Q_{12}+\left[B_{11}(\alpha, \beta, \xi)-B_{22}(\alpha, \beta, \xi)\right] Q_{12} \\
& -B_{21}(\alpha, \beta, \xi) Q_{12}^{2}+B_{12}(\alpha, \beta, \xi), \\
a(\alpha, \beta, \xi) \cdot \xi \frac{d Q_{21}}{d \xi}= & \left(\lambda_{2}-\lambda_{1}\right) Q_{21}+\left[B_{22}(\alpha, \beta, \xi)-B_{11}(\alpha, \beta, \xi)\right] Q_{21} \\
& -B_{12}(\alpha, \beta, \xi) Q_{21}^{2}+B_{21}(\alpha, \beta, \xi)
\end{aligned}
$$

which define the functions $Q_{12}$ and $Q_{21}$ respectively.

\section{$3^{\circ}$. Nonlinear problem.}

Therefore, in order to study the equations (72.11) and (72.12), it is sufficient to study a nonlinear ordinary differential equation :

$$
\mathfrak{a}(\alpha, \beta, \xi) \cdot \xi \frac{d Q}{d \xi}=\lambda\left(\alpha^{l^{\prime}} \beta^{m^{\prime}} \xi^{n^{\prime}}, \alpha^{l^{\prime \prime}} \beta^{m^{\prime \prime}} \xi^{n^{\prime \prime}}\right) Q+f(\alpha, \beta, \xi ; Q)+g(\alpha, \beta, \xi) .
$$

Here $\lambda(\eta, \zeta)$ is a function holomorphic and bounded in $(\eta, \zeta)$ for the domain (72.6) and satisfies the inequality there

$$
|\lambda(\eta, \zeta)| \geqq \omega>0,
$$

where $\omega$ is a constant independent of $\eta$ and $\zeta ; f$ has a form

$$
f(\alpha, \beta, \xi ; Q)=\sum_{h=1}^{\infty} Q^{h} f_{h}(\alpha, \beta, \xi),
$$

where the power series in the right-hand member is uniformly convergent for



$g(\alpha, \beta, \xi)$ and $f_{h}(\alpha, \beta, \xi)$ are functions with properties similar to the matrix $A(\alpha, \beta, \xi)$; especially, we have the relation

for (72.14).

$$
|g(\alpha, \beta, \xi)|,\left|f_{1}(\alpha, \beta, \xi)\right|=O\left(\left|\alpha^{l} \beta^{m} \xi^{n}\right|\right)
$$

\section{$4^{\circ}$. Formal solution of (72.13). \\ Put}

$$
Q(\alpha, \beta, \xi) \sim \sum_{h=1}^{\infty}\left(\alpha^{l} \beta^{m} \xi^{n}\right)^{h} Q_{h}\left(\alpha^{l^{\prime}} \beta^{m^{\prime}} \xi^{n^{\prime}}, \alpha^{l^{\prime \prime}} \beta^{m^{\prime \prime}} \xi^{n^{\prime \prime}}\right),
$$

where we impose upon the coefficients $Q_{h}(\eta, \zeta)$ the following conditions:

$Q_{h}(\eta, \zeta)$ are functions with the same asymptotic behavior as that of the matrix functions $A_{h}(\eta, \zeta)$ in the domain (72.6).

If we put the formal power series (72.15) into both sides of (72.13), we obtain a formal equality. Equating the coefficients of the term $\left(\alpha^{l} \beta^{m} \xi^{n}\right)^{N}$, we can easily derive the following equation:

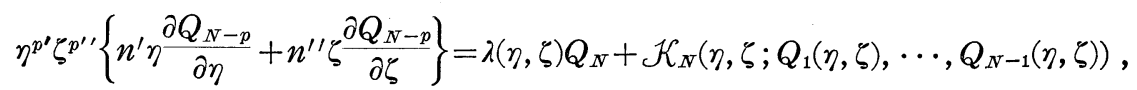


where $\mathcal{K}_{N}\left(\eta, \zeta, q_{1}, \cdots, q_{N-1}\right)$ is a polynomial of $q_{1}, \cdots, q_{N-1}$ whose coefficients are functions with the properties similar to the matrices $A_{h}(\eta, \zeta)$ appeared in (72.3).

Suppose that the functions $Q_{h}(\eta, \zeta)(h<N)$ with the desired property have been already determined. Then, since $p>0$, the terms appearing in (72.16) except for the expression $\lambda(\eta, \zeta) Q_{N}$ will be regarded as known functions. Further, the function $\lambda(\eta, \zeta)$ is supposed to be non-vanishing in the domain (72.6). Therefore, we get immediately from (72.16) the function $Q_{N}(\eta, \zeta)$ with the desired property.

\section{$5^{\circ}$. Analytical menaing (Asymptotic developability).}

Finally we must give an analytical meaning to such a formal solution.

To do this, put

$$
Q^{(N)}(\alpha, \beta, \xi)=\sum_{h=1}^{N-1}\left(\alpha^{l} \beta^{m} \xi^{n}\right)^{h} Q_{h}\left(\alpha^{l^{\prime}} \beta^{m^{\prime}} \xi^{n^{\prime}}, \alpha^{l^{\prime \prime}} \beta^{m^{\prime \prime}} \xi^{n^{\prime \prime}}\right)
$$

and let us make following two transformations successively:

$$
\begin{aligned}
& Q=Q^{(N)}(\alpha, \beta, \xi)+R, \\
& R=S \exp \Lambda(\alpha, \beta, \xi),
\end{aligned}
$$

where

$$
\Lambda(\alpha, \beta, \xi)=\int_{\xi^{*}}^{\xi} \frac{\lambda\left(\alpha^{l^{\prime}} \beta^{m^{\prime}} \xi^{n^{\prime}}, \alpha^{l^{\prime \prime}} \beta^{m^{\prime \prime}} \xi^{n^{\prime \prime}}\right)}{a(\alpha, \beta, \xi) \cdot \xi} d \xi,
$$

$\xi^{*}$ being a certain boundary point.

Then, it is easily verified that the equation (72.13) is transformed into an equation of the form

$$
\frac{d S}{d \xi}=a(\alpha, \beta, \xi)^{-1} \xi^{-1} F\left(\alpha, \beta, \xi ; S e^{\Lambda(\alpha, \beta, \xi)}\right) e^{-\Lambda(\alpha, \beta, \xi)},
$$

where $F(\alpha, \beta, \xi ; R)$ is a function holomorphic and bounded in $(\alpha, \beta, \xi, R)$ for

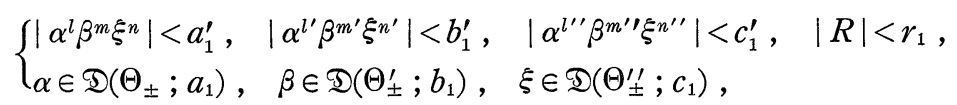

and, moreover, satisfies a following inequality

$$
|F(\alpha, \beta, \xi ; R)| \leqq L\left|\alpha^{l} \beta^{m} \xi^{n}\right||R|+L_{N}\left|\alpha^{l} \beta^{m} \xi^{n}\right|^{N} ;
$$

$L$ is a positive constant independent of $\alpha, \beta, N$, while $L_{N}>0$ may depend on $N$ only.

Let

$$
\alpha \in \mathfrak{D}\left(\Theta_{0} \pm \gamma ; \infty\right), \quad \beta \in \mathscr{D}\left(\Theta_{0}^{\prime} \pm \gamma^{\prime} ; \infty\right), \quad \xi \in \mathfrak{D}\left(\Theta_{0}^{\prime \prime}+\gamma^{\prime \prime} ; \infty\right)
$$

be an arbitrary proper region with respect to the function (72.17) (See $\S 71$ concerning the definition of proper region). Put

$$
\begin{cases}\widetilde{\Theta}_{+}=\min \left(\Theta_{+}, \Theta_{0}+\gamma\right), & \widetilde{\Theta}_{-}=\max \left(\Theta_{-}, \Theta_{0}-\gamma\right) ; \\ \widetilde{\Theta}_{+}^{\prime}=\min \left(\Theta_{+}^{\prime}, \Theta_{0}^{\prime}+\gamma^{\prime}\right), & \widetilde{\Theta}_{-}^{\prime}=\max \left(\Theta_{-}^{\prime}, \Theta_{0}^{\prime}-\gamma^{\prime}\right) ; \\ \widetilde{\Theta}_{+}^{\prime \prime}=\min \left(\Theta_{+}^{\prime \prime}, \Theta_{0}^{\prime \prime}+\gamma^{\prime \prime}\right), & \widetilde{\Theta}_{-}^{\prime \prime}=\max \left(\Theta_{-}^{\prime \prime}, \Theta_{0}^{\prime \prime}-\gamma^{\prime \prime}\right) .\end{cases}
$$

In order to prove the asymptotic developability of a formal solution 
obtained in $4^{\circ}$, it is sufficient to prove the following proposition :

The equation (72.18) admits a unique solution $S=S_{N}(\alpha, \beta, \xi)$, where $S_{N}(\alpha, \beta, \xi)$ is a function holomorphic and bounded in $(\alpha, \beta, \xi)$ for

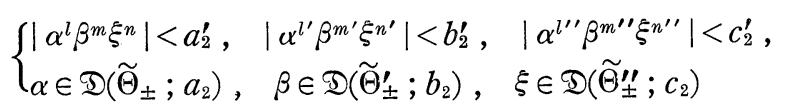

and satisfies a following inequality there

$$
\left|S_{N}(\alpha, \beta, \xi)\right| \leqq K_{N}\left|\alpha^{l} \beta^{m} \xi^{n}\right|^{N} \exp \{-\Re \Lambda(\alpha, \beta, \xi)\}
$$

with a boundary condition

$$
S_{N}\left(\alpha, \beta, \xi^{*}\right)=s_{N}(\alpha, \beta),
$$

where $K_{N}$ is a positive constant independent of $\alpha, \beta ; s_{N}(\alpha, \beta)$ is a holomorphic and bounded function of $\alpha, \beta$ in the domain (72.20) (where we have to put $\xi=\xi^{*}$ ) and satisfies the inequality

$$
\left|s_{N}(\alpha, \beta)\right| \leqq K_{N}\left|\alpha^{l} \beta^{m} \xi^{* n}\right|^{N} .
$$

It is noticed that:

1) Since $(l, m, n),\left(l^{\prime}, m^{\prime}, n^{\prime}\right),\left(l^{\prime \prime}, m^{\prime \prime}, n^{\prime \prime}\right)$ are supposed to be the directional parameters of the three edges of a trigonal pyramid containing the first octant of $(X, Y, Z)$-space in its interior, at least one of $n, n^{\prime}, n^{\prime \prime}$ is positive :

2) Therefore, the inequalities

$$
\left|\alpha^{l} \beta^{m} \xi^{n}\right|<a_{2}^{\prime}, \quad\left|\alpha^{l^{\prime}} \beta^{m^{\prime}} \xi^{n^{\prime}}\right|<b_{2}^{\prime}, \quad\left|\alpha^{l^{\prime \prime}} \beta^{m^{\prime \prime}} \xi^{n^{\prime \prime}}\right|<c_{2}^{\prime}
$$

appearing in (72.20) are equivalent to

$$
\rho(\alpha, \beta)<|\xi|<\kappa(\alpha, \beta), \quad \rho(\alpha, \beta)<\kappa(\alpha, \beta),
$$

where $\rho(\alpha, \beta)$ and $\kappa(\alpha, \beta)$ depend on $\left.\alpha, \beta .^{6}\right)$

Then, the proof of the proposition is carried out in quite a similar way as in [5].

Thus the functions $Q_{12}(\alpha, \beta, \xi)$ and $Q_{21}(\alpha, \beta, \xi)$ with the desired property have been determined. By substituting these two functions into the first two relations of $(72.10)^{\prime}$, the functions $\Lambda_{i}(\alpha, \beta, \xi)(i=1,2)$ are also determined.

This proves the lemmas 6 and 7 .

\section{$\S 73$. Remark on a system of Type $\mathbf{A}^{\prime}$.}

We have remarked, at the end of Chapter III, that we must study a system of Type $A^{\prime}$ in a certain case :

$$
\left(\alpha^{l^{\prime}} \beta^{m^{\prime}} \xi^{n^{\prime}}\right) p^{p^{\prime}}\left(\alpha^{l^{\prime \prime}} \beta^{m^{\prime \prime}} \xi^{n^{\prime \prime}}\right)^{p^{\prime \prime}} \cdot \xi \frac{d \overrightarrow{\mathfrak{w}}}{d \xi}=A(\alpha, \beta, \xi) \overrightarrow{\mathfrak{w}},
$$

where at least one of $p^{\prime}$ and $p^{\prime \prime}$ is positive and $A(\alpha, \beta, \xi)$ is a matrix function with the same asymptotic behavior as in Type A :

6) For example, if $n \leqq 0, n^{\prime}>0, n^{\prime \prime}>0$, it is sufficient to put

$$
\left\{\begin{array}{l}
\rho(\alpha, \beta)=\left[a_{2}^{\prime-1} \alpha^{l} \beta^{m}\right]^{-1 / n}(n \neq 0) \text { or } 0(n=0), \\
\kappa(\alpha, \beta)=\min \left\{\left[b_{2}^{\prime-1} \alpha^{l^{\prime}} \beta^{m^{\prime}}\right]^{-1 / n^{\prime}},\left[c_{2}^{\prime-1} \alpha^{l^{\prime \prime}} \beta^{m^{\prime \prime}}\right]^{-1 / n^{\prime \prime}}\right\} .
\end{array}\right.
$$




$$
\begin{aligned}
& A(\alpha, \beta, \xi) \sim \sum_{h=0}^{\infty}\left(\alpha^{l} \beta^{m} \xi^{n}\right)^{h} A_{h}\left(\alpha^{l^{\prime}} \beta^{m^{\prime}} \xi^{n^{\prime}}, \alpha^{l^{\prime \prime}} \beta^{m^{\prime \prime}} \xi^{n^{\prime \prime}}\right), \\
& A_{h}(\eta, \zeta) \sim \sum_{k=0}^{\infty} \eta^{k} A_{h k}(\zeta) \\
& A_{h k}(\zeta) \quad \sim \sum_{j=0}^{\infty} \zeta^{j} A_{h k j} .
\end{aligned}
$$

In this case also, we can construct a formal transformation

$$
\begin{gathered}
\overrightarrow{\mathfrak{w}}=P(\alpha, \beta, \xi) \overrightarrow{\mathfrak{v}}, \\
P \sim \sum_{h=0}^{\infty}\left(\alpha^{l} \beta^{m n} \xi\right)^{h} P_{h}\left(a^{l^{\prime}} \beta^{m^{\prime}} \xi^{n^{\prime}}, \alpha^{l^{\prime \prime}} \beta^{m^{\prime \prime}} \xi^{n^{\prime \prime}}\right),
\end{gathered}
$$

such that the coefficient matrix of the formally transformed system is formally diagonalized,

Concerning an analytical meaning of such a formal transformation, the lemma 6 or 7 holds according as $n^{\prime} p^{\prime}+n^{\prime \prime} p^{\prime \prime} \neq 0$ or not.

In this section we will explain the outline of constructing such a formal transformation.

$\mathbf{1}^{\circ}$. By making a linear transformation with constant coefficients

$$
\overrightarrow{\mathfrak{w}}=P_{000} \overrightarrow{\mathfrak{w}}_{1} \text {, }
$$

we assert that the matrix $A_{000}$ has a diagonal form. We denote by $\lambda_{j}$ the diagonal elements.

$\mathbf{2}^{\circ}$. Let

$$
\alpha \in \mathscr{D}\left(\Theta_{0} \pm \gamma ; \infty\right), \quad \beta \in \mathscr{D}\left(\Theta_{0}^{\prime} \pm \gamma^{\prime} ; \infty\right), \quad \xi \in \mathscr{D}\left(\Theta_{0}^{\prime \prime} \pm \gamma^{\prime \prime} ; \infty\right)
$$

be an arbitrary proper region with respect to the functions (71.8) or (71.8) (where we have to put $p=0$ ) according as $n^{\prime} p^{\prime}+n^{\prime \prime} p^{\prime \prime} \neq 0$ or not. In the latter case, we may take $\Theta_{0}^{\prime \prime} \pm \gamma^{\prime \prime}=\Theta_{ \pm}^{\prime \prime}$. Put

or

$$
\begin{cases}\widetilde{\Delta}_{+}^{\prime \prime}=\min \left\{\max _{\alpha} \arg \left(\alpha^{l^{\prime \prime}} \beta^{m^{\prime \prime}} \xi^{n^{\prime \prime}}\right),\right. & \left.\Delta_{+}^{\prime \prime}\right\}, \\ \widetilde{\Delta}_{-}^{\prime \prime}=\max \left\{\underset{\alpha \beta, \xi}{\left\{\min _{\beta}\right.} \arg \left(\alpha^{l^{\prime \prime}} \beta^{m^{\prime \prime}} \xi^{n^{\prime \prime}}\right),\right. & \left.\Delta_{-}^{\prime \prime}\right\}\end{cases}
$$

$$
\left\{\begin{array}{l}
\widetilde{\Delta}_{+}^{\prime \prime}=\Delta_{+}^{\prime \prime}, \\
\widetilde{\Delta}_{-}^{\prime \prime}=\Delta_{-}^{\prime \prime}
\end{array}\right.
$$

according as $p^{\prime}=0$ or not, where $(\alpha, \beta, \xi)$ is contained in (73.2).

Then we can prove the following:

There exists a formal transformation

$$
\overrightarrow{\mathfrak{w}}_{1}=P_{00}\left(\alpha^{l \prime \prime} \beta^{m^{\prime \prime}} \xi^{n^{\prime \prime}}\right) \overrightarrow{\mathfrak{w}}_{2}
$$

such that the matrix function corresponding to $A_{00}(\zeta)$ (of. the formally transformed system) is reduced to a diagonal matrix with diagonal elements $\lambda_{i}(\zeta)(i=1,2)$ such that $\lim _{\zeta \rightarrow 0} \lambda_{i}(\zeta)=\lambda_{i}$, where $P_{00}(\zeta)$ is a nonsingular matrix function holomorphic, bounded and asymptotically developable in powers of $\zeta$ :

$$
P_{00} \sim 1+\sum_{j=0}^{\infty} \zeta^{j} P_{00 j},
$$


where 1 is a unit-matrix, as $\zeta$ tends to 0 in a domain

$$
\zeta \in \mathfrak{D}\left(\widetilde{\Delta}_{ \pm}^{\prime \prime} ; d_{1}^{\prime \prime}\right) \text {. }
$$

$3^{\circ}$. Put

$$
\begin{cases}\widetilde{\Delta}_{+}^{\prime}=\min \left\{\max _{\alpha, \beta, \xi} \arg \left(\alpha^{l^{\prime}} \beta^{m^{\prime}} \xi^{n^{\prime}}\right),\right. & \left.\Delta_{+}^{\prime}\right\}, \\ \widetilde{\Delta}_{-}^{\prime}=\max \left\{\min _{\alpha, \beta, \xi} \arg \left(\alpha^{l^{\prime}} \beta^{m^{\prime}} \xi^{n^{\prime}}\right),\right. & \left.\Delta_{-}^{\prime}\right\},\end{cases}
$$

where $(\alpha, \beta, \xi)$ is contained in (73.2).

Let $P_{0}(\eta, \zeta)$ be a matrix function which is nonsingular, holomorphic and bounded in $(\eta, \zeta)$ for

$$
\eta \in \mathfrak{D}\left(\Delta_{ \pm}^{\prime} ; d_{1}^{\prime}\right), \quad \zeta \in \mathfrak{D}\left(\Delta_{ \pm}^{\prime \prime} ; d_{1}^{\prime \prime}\right)
$$

and admits a uniform asymptotic expansion in powers of $\eta$ :

$$
P_{0}(\eta, \zeta) \sim 1+\sum_{k=1}^{\infty} \eta^{k} P_{0 k}(\zeta)
$$

as $\eta$ tends to 0 in the domain (73.4), where $P_{0 k}(\zeta)$ are matrix functions with the same asymptotic behavior as the matrix $P_{00}(\zeta)$ appeared in $2^{\circ}$.

Then we can prove the following:

There exists a formal transformation

$$
\overrightarrow{\mathfrak{w}}_{2}=P_{0}\left(\alpha^{l^{\prime}} \beta^{m^{\prime}} \xi^{n^{\prime}}, \alpha^{l^{\prime \prime}} \beta^{m^{\prime \prime}} \xi^{n^{\prime \prime}}\right) \overrightarrow{\mathfrak{w}}_{3},
$$

where $P_{0}(\eta, \zeta)$ is a matrix function satisfying the conditions given above, by which the matrix function corresponding to $A_{0}(\eta, \zeta)$ (of the formally transformed system) is reduced to a diagonal form whose elements are given by $\lambda_{i}(\eta, \zeta)$ such that $\lim _{\eta \rightarrow 0} \lambda_{i}(\eta, \zeta)=\lambda_{i}(\zeta)$.

$4^{\circ}$. Let $Q_{h}(\eta, \zeta)$ be matrix functions holomorphic and bounded in $(\eta, \zeta)$ for (73.4) and possessing the same asymptotic behavior as the matrix function $P_{0}(\eta, \zeta)$.

Then, we can prove the following:

There exists a formal transformation

$$
\begin{gathered}
\overrightarrow{\mathfrak{m}}_{3}=Q(\alpha, \beta, \xi) \overrightarrow{\mathfrak{y}}, \\
Q \sim \mathbf{1}+\sum_{h=1}^{\infty}\left(\alpha^{l} \beta^{m} \xi^{n}\right)^{h} Q_{h}\left(\alpha^{l^{\prime}} \beta^{m^{\prime}} \xi^{n^{\prime}}, \alpha^{l^{\prime \prime}} \beta^{m^{\prime \prime}} \xi^{n^{\prime \prime}}\right),
\end{gathered}
$$

where $Q_{h}(\eta, \zeta)$ are matrix functions satisfying the conditions given above, such that the matrix functions corresponding to $A_{h}(\eta, \zeta)$ (of the formally transformed system) are all reduced to diagonal matrices.

Therefore, to obtain a formally diagonalizing transformation, it is sufficient to compose the formal transformations obtained in $1^{\circ} \sim 4^{\circ}$ :

$$
\overrightarrow{\mathfrak{w}}=P(\alpha, \beta, \xi) \overrightarrow{\mathfrak{v}},
$$

where

$$
P=P_{000} P_{00}\left(\alpha^{l^{\prime \prime}} \beta^{m^{\prime \prime}} \xi^{n^{\prime \prime}}\right) P_{0}\left(\alpha^{l^{\prime}} \beta^{m^{\prime}} \xi^{n^{\prime}}, \alpha^{l^{\prime \prime}} \beta^{m^{\prime \prime}} \xi^{n^{\prime \prime}}\right) Q(\alpha, \beta, \xi) .
$$

Clearly, this matrix function is formally developable in the form (73.1) whose coefficients $P_{k}(\eta, \zeta)$ are matrix functions holomorphic and bounded in $(\eta, \zeta)$ for $(73.4)$ and admitting uniform asymptotic expansions : 


$$
P_{h}(\eta, \zeta) \sim \sum_{k=0}^{\infty} \eta^{k} P_{h k}(\zeta)
$$

as $\eta$ tends to 0 in the domain (73.4), where $P_{h k}(\zeta)$ are matrix functions holomorphic, bounded and asymptotically developable in powers of $\zeta$ for (73.3).

\section{References}

[1] A. Erdélyi and C. A. Swanson, Asymptotic forms of Whittaker's confluent hypergeometric functions. Memoirs Amer. Math. Soc. No. 25 (1957).

[ 2 ] M. Hukuhara, Sur les propriétés asymptotiques des solutions d'un système d'équations différentielles linéaires contenant un paramètre. Mem. Fac. Engin. Kyushu Univ. 8 (1937), 249-280.

[3] M. Iwano, Asymptotic solutions of a system of linear ordinary differential equations containing two parameters (in japanese). Funkcialaj Ekvacioj (Internal), 14 (1961) N-ro 1, 11-51.

[4] M. Iwano, Asymptotic solutions of a system of linear ordinary differential equations containing a small parameter, I. Funkcialaj Ekvacioj 5 (1963), 71-134.

[5] M. Iwano, Asymptotic solutions of a system of linear ordinary differential equations containing a small parameter, II (to appear soon).

[6] M. Iwano and Y. Sibuya, Reduction of the order of a linear ordinary differential equation containing a small parameter. Kōdai Math. Semi. Rep. 15 (1963) 1-28.

[ 7] K. Takahasi, Über eine erweiterte asymptotische Darstellung der Lösung eines systems von homogenen linearen Differentialgleichungen, welche von zwei Parametern abhängen. Jour. Fac. Sci. Univ. Tokyo, I, 8 (1956), 1-73. 